九州大学学術情報リポジトリ

Kyushu University Institutional Repository

\title{
RANDOM COMBINED FRACTIONAL FACTORIAL DESIGNS II : SAMPLING THEORY FROM THE FINITE POPULATION
}

Yamakawa, Norihiro

Kasado Works, Hitachi Ltd.

https://doi.org/10.5109/13018

出版情報 : 統計数理研究. 12 (1/2)，pp.1-67，1966-03. Research Association of Statistical Sciences バージョン :

権利関係 : 


\title{
Random Combined Fractional Factorial Designs II : Sampling theory from the Finite Population
}

By

\author{
Norihiro YAMAKAWA* \\ (Received November 25, 1964)
}

\section{Introduction}

Let us consider the following randomization procedures in our designs (refer to Literature [12]).

(1) We have a design matrix

$$
D=\begin{gathered}
D_{P} \\
D_{\circledast} \\
D_{R}
\end{gathered}
$$

which is composed of three sub-matrices

$$
\begin{aligned}
& \begin{array}{llll}
x_{11} & x_{12} & \cdots \cdots & x_{1 N}
\end{array} \\
& D_{P}=\begin{array}{lllll}
x_{21} & x_{22} & \cdots \cdots & x_{2 N}
\end{array} \\
& \begin{array}{llll}
x_{P 1} & x_{P 2} & \cdots & x_{P N}
\end{array}
\end{aligned}
$$

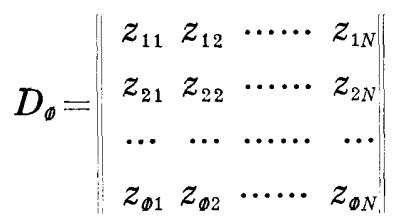

and

$$
D_{R}=\left\|\begin{array}{cccc}
\xi_{1 \pi(1)} & \xi_{1 \pi(2)} & \cdots \cdots & \xi_{1 \pi(N)} \\
\xi_{2 \pi(1)} & \xi_{2 \pi(2)} & \cdots \cdots & \xi_{2 \pi(N)} \\
\cdots & \cdots & \cdots \cdots & \cdots \\
\xi_{R \pi(1)} & \xi_{R \pi(2)} & \cdots \cdots & \xi_{R \pi(N)}
\end{array}\right\| .
$$

(2) In these sub-matrices, we have

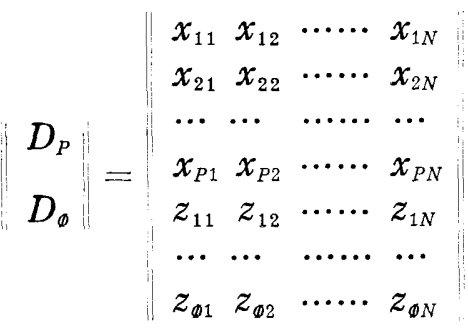

* Kasado Works, Hitachi Ltd 
as an orthogonal array of size

$$
N=P+\Phi
$$

with two levels

$$
\left\{\begin{array}{lll}
x_{p n}=+1 & \text { or } & -1 \\
z_{\phi n}=+1 & \text { or } & -1
\end{array} .\right.
$$

(3) With equal probability $1 / N$ !, we choose a permutation

$$
\Pi=\left(\begin{array}{cccc}
1 & 2 & \cdots \cdots & N \\
\pi(1) & \pi(2) & \cdots \cdots & \pi(N)
\end{array}\right)
$$

from the set of all possible permutations of $N$ numbers $1,2, \cdots, N-1$ and $N$.

In virtue of these randomization procedure, the variable

$$
\dot{V}_{p}^{\prime}=\sum_{n=1}^{N} x_{n p} \quad v_{\pi(n)}^{\prime} / N
$$

$$
(p=1,2, \cdots, P)
$$

becomes a random variable, where

$$
v_{\pi(n)}^{\prime}=\sum_{r=1}^{R} \beta_{r}^{\prime} \xi_{r \pi(n)}
$$

$$
(n=1,2, \cdots, N)
$$

and $\beta_{1}^{\prime}, \beta_{2}^{\prime}, \cdots, \beta_{R}^{\prime}$ are parameters. The purpose of this paper is to discuss the distribution of the random variable $\dot{V}_{p}^{\prime}$.

\section{A sampling distribution function from a finite population}

\subsection{Direct evaluation of probability distribution}

First of all, let us consider the distribution function of statistics introduced from a classification of a finite population, $v_{1}, v_{2}, \cdots, v_{N}$, which can be classified into $K(<N)$ classes such that $N P_{1}$ eiements of $\bar{v}_{1}, N P_{2}$ elements of $\bar{v}_{2}, \cdots, N P_{k}$ elements of $\bar{v}_{k}, \cdots, N P_{K}$ elements of $\bar{v}_{K}$ where $\bar{v}_{k}$ $(k=1,2, \cdots, K)$ is the value of $v_{n}$ in the $k$-th class.

These $N$ points of $v_{n}(n=1,2, \cdots, N)$ which randomly correspond to the $N$ columns in the design matrix $(0.1)$ by our randomization procedure are divided into 2 groups by an arbitrary $p$-th row in O.A. such that +1 of $x_{p n}$ group contains $N_{1}=\frac{N}{2}$ points and -1 of $x_{p n}$ group contains $N_{2}=\frac{N}{2}$ points, where suffix " 1 " stands for " + " $\operatorname{sign}$ group and suffix " 2 " stands for "- " sign group.

In these circumstances, $N$ elements in the sequence $\left\{v_{n}\right\}$ are divided into two groups, such that the 1 st group of these is the sequence $\left\{\dot{u}_{k 1}\right\}(k$ $=1,2, \cdots, K)$ and the 2 nd group of these is the sequence $\left\{\dot{u}_{k 2}\right\} \quad(k=1,2$, $\cdots, K)$ in which $\dot{u}_{k 1}$ and $\dot{u}_{k 2}$ are the number of $k$-th class in the 1 st and 
2nd groups respectively. Then we have $\sum_{k=1}^{K} \dot{u}_{k i}=N_{i}, \quad(i=1,2)$ and $\sum_{i=1}^{2} \dot{u}_{k i}=N P_{k}, \quad(k=1,2, \cdots, K)$. Consequently, we get

$$
\begin{aligned}
\dot{V}_{p} & =\frac{1}{N} \sum_{n=1}^{N} \dot{v}_{\pi(n)} x_{p n} \\
& =\frac{2}{N} \sum_{k=1}^{K} v_{k} \dot{u}_{k 1},
\end{aligned}
$$

where we used the relation $\frac{1}{N} \sum_{n=1}^{N} \dot{v}_{\pi(n)}=\frac{1}{N} \sum_{r=1}^{R} \beta_{r} \sum_{n=1}^{N} \xi_{r \pi(n)}=0$. Then we get the probability, that for any assigned set $b_{11}, \cdots, b_{K 1}$, we have $\dot{u}_{11}, \cdots, \dot{u}_{K 1}=$ $b_{11}, \cdots, b_{K 1}$, is

$$
P_{r}\left(b_{11}=\dot{u}_{11}, \cdots, b_{K 1}=\dot{u}_{K 1}\right)=\prod_{k=1}^{K}\left(\begin{array}{c}
N P_{k} \\
b_{k 1}
\end{array}\right) /\left(\begin{array}{l}
N \\
N_{1}
\end{array}\right) \text {. }
$$

For any assigned set of $\left\{b_{k \mu}\right\} k=1,2, \cdots, K$, so as to simplify the following discussions, we shall denote the joint probability function of the random variables $\dot{u}_{1 \mu}=b_{1 \mu}, \dot{u}_{2 \mu}=b_{2 \mu}, \cdots, \dot{u}_{k \mu}=b_{k \mu}$ as $P\left\{\prod_{k=1}^{K}\left(\dot{u}_{k \mu}=b_{k \mu}\right)\right\} \quad$ (for any $\mu$ ) and $P\left\{\prod_{k=1}^{K} \prod_{\mu=1}^{M}\left(\dot{u}_{k \mu}=b_{k \mu}\right)\right\}$, where $\mu$ means the suffix representing the classification number such as $i j \cdots k_{0}(i, j, \cdots, k=1,2)$. Furthermore, we shall use the notation $b_{k \mu}$ for the assigned value corresponding random variable $\dot{u}_{k \mu}$.

Secondly, let us consider the case that $N$ points of $v_{\pi(n)}$ are divided into $2^{2}=4$ groups by two arbitrary $p$ and $q$-th rows in O. A. such that $\{+1$, $+1\}$ group contains $N_{11}=N / 4$ points $\{+1,-1\}$ gronp contains $N_{12}=N / 4$ points $\{-1,+1\}$ group contains $N_{21}=N / 4$ points $\{-1,-1\}$ group contains $N_{22}=N / 4$ points where suffices " 1 " and " 2 " stand for " + " and " - " sign group, respectively, then " 11 " stands for " ++ " sign group, and so on.

In these circumstances, $N$ elements in the sequence $\left\{v_{n}\right\} \quad(n=1,2, \cdots$, $N)$ are classified in the two-way classification as following page.

In Table 1.1.1 and in later discussions, we shall denote the sign " 0 " so as to show the suffix with which we have operate the summation as follows, $\quad \sum_{i=1}^{2} \dot{u}_{k i j}=\dot{u}_{k 0 j}, \quad \sum_{i=1}^{2} b_{k i j}=b_{k 0 j}$, and $\sum_{j=1}^{2} \dot{u}_{k i j}=\dot{u}_{k i 0}, \quad \sum_{j=1}^{2} b_{k i j}=b_{k i 0}$.

The conditional probability, that for any assigned set of $\left\{b_{k 11}\right\},\left\{\dot{u}_{k 11}\right\}$ $(k=1,2, \cdots, K)$ elements are contained in $N_{11}$ random samples from the population $N_{10}=\sum_{j=1}^{2} N_{i j}$ under the condition that for any assigned set of $\left\{b_{k 10}\right\},\left\{u_{k 10}\right\}(k=1,2, \cdots, K)$ elements are contained in $N_{10}$ random samples from the grand population $N$, is obtained from the expression 
Table 1.1.1 Two-way classification table of $\left\{v_{n}\right\}$

\begin{tabular}{|c|c|c|c|c|c|c|}
\hline$p^{q}$ & & $\frac{1}{t}$ & & - & & Sum \\
\hline+ & $\begin{array}{c}\dot{u}_{111} \\
\dot{u}_{211} \\
\ldots \\
\dot{u}_{K 11}\end{array}$ & $N_{11}$ & $\begin{array}{c}\dot{u}_{112} \\
u_{212} \\
\vdots \\
\dot{u}_{K 12}\end{array}$ & $N_{12}$ & $\begin{array}{c}\dot{u}_{110} \\
\dot{u}_{210} \\
\vdots \\
\dot{u}_{K 10}\end{array}$ & $N_{10}$ \\
\hline- & $\begin{array}{c}\dot{u}_{121} \\
u_{u_{221}} \\
\vdots \\
\dot{u}_{K 21}\end{array}$ & $N_{21}$ & $\begin{array}{c}\dot{u}_{122} \\
\dot{u}_{222} \\
\vdots \\
\dot{u}_{K 22}\end{array}$ & $N_{22}$ & $\begin{array}{c}\dot{u}_{120} \\
\dot{u}_{220} \\
\vdots \\
\dot{u}_{K 02}\end{array}$ & $N_{20}$ \\
\hline Sum & $\begin{array}{c}\dot{u}_{101} \\
\dot{u}_{201} \\
\vdots \\
\dot{u}_{K 01}\end{array}$ & $N_{01}$ & $\begin{array}{c}\dot{u}_{102} \\
\dot{u}_{202} \\
\vdots \\
\dot{u}_{K 02}\end{array}$ & $N_{02}$ & $\begin{array}{c}N P_{1} \\
N P_{2} \\
\vdots \\
N P_{K}\end{array}$ & $N$ \\
\hline
\end{tabular}

Similarly, the conditional probability, that $\left\{b_{k 21}\right\}(k=1,2, \cdots, K)$ elements are containing in $N_{21}$ random samples from the population $N_{20}$ under the condition that $\left\{b_{k 20}\right\}(k=1,2, \cdots, K)$ elements are containing in $N_{20}$ random samples from the grand population $N$, is obtained from the expression

$$
P\left\{\prod_{k=1}^{K}\left(\dot{u}_{k 21}=b_{k 21}\right) \prod_{k=1}^{K}\left(\dot{u}_{k 20}=b_{k 20}\right)\right\}=\prod_{k=1}^{K}\left(\begin{array}{l}
b_{k 20} \\
b_{k 21}
\end{array}\right) /\left(\begin{array}{l}
N_{20} \\
N_{21}
\end{array}\right) .
$$

Since these two random sequences $\left\{\dot{u}_{k 11}\right\}$ and $\left\{\dot{u}_{k 12}\right\} \quad(k=1,2, \cdots, K)$ are mutually independently distributed, then we have the probability that the sequences $\left\{b_{k 11}\right\}$ and $\left\{b_{k 21}\right\}(k=1,2, \cdots, K)$ are jointly sampled, such that

$$
\begin{gathered}
P\left\{\prod_{k=1}^{K}\left(\dot{u}_{k 11}=b_{k 11}\right) \prod_{k=1}^{K}\left(\dot{u}_{k 21}=b_{k 21}\right) \quad \prod_{k=1}^{K}\left(\dot{u}_{k 10}=b_{k 10}\right)\right\} \\
=\prod_{k=1}^{K}\left(\begin{array}{l}
b_{k 10} \\
b_{k 11}
\end{array}\right)\left(\begin{array}{l}
b_{k 20} \\
b_{k 21}
\end{array}\right)\left(\begin{array}{l}
N_{10} \\
N_{11}
\end{array}\right)\left(\begin{array}{l}
N_{20} \\
N_{21}
\end{array}\right) .
\end{gathered}
$$

Consequently, we get the joint probability distribution

$$
\begin{aligned}
& P\left\{\prod_{k=1}^{K}\left(\dot{u}_{k 11}=b_{k 11}, \dot{u}_{k 21}=b_{k 21}, \dot{u}_{k 10}=b_{k 10}\right)\right\} \\
& \quad=\prod_{k=1}^{K} \frac{\left(N P_{k}\right) !}{\left(b_{k 11}\right) !\left(b_{k 12}\right) !\left(b_{k 21}\right) !\left(b_{k 22}\right) !} / \frac{N !}{N_{11} ! N_{12} ! N_{21} ! N_{22} !} .
\end{aligned}
$$

Furthermore, in the case that $N$ points of complete orthogonal array is equal to $N=2^{J}$, we can classify the $N$ elements of the sequence $\left\{v_{n}\right\}(n=$ $1,2, \cdots, N$ ) into $N_{\underbrace{11 \cdot 1}_{J}}, \underbrace{N_{11 \cdots \cdots}}_{J}, \cdots, \underbrace{N_{22 \cdots 2}}_{J}$ elements, respectively, in a similar manner as following Table. In other words, we can represent these $J$-way classification in the following Figure. 
Table 1.1.2 $J$-way $\left(2^{J}=N\right)$ classification table

\begin{tabular}{|c|c|c|c|c|c|c|}
\hline & $p_{1} p_{2} \cdots \cdots \cdots \cdots p_{J}$ & & 1 & 2 & $\cdots \cdots$ & $K$ \\
\hline 1 & $11 \ldots \ldots \ldots 1$ & $N_{J}^{\underbrace{11 \cdots 1}}$ & $\dot{u}_{111 \ldots 1} \underbrace{}_{J}$ & $\dot{u}_{211 \cdots 1}$ & & $\dot{u}_{K} \underbrace{11 \cdots 1}_{J}$ \\
\hline 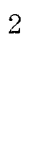 & 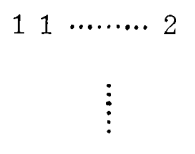 & $\begin{array}{c}N_{J}^{N_{11 \cdots 2}} \\
\vdots \\
\vdots\end{array}$ & $\begin{array}{c}\dot{u}_{111 \cdots 2} \\
\vdots \\
\vdots\end{array}$ & $\begin{array}{c}\dot{u}_{211 \cdots 2} \\
\vdots \\
\vdots\end{array}$ & $\ldots \ldots$ & $\begin{array}{c}\dot{u}_{K 11 \cdots 2} \\
\vdots \\
\vdots\end{array}$ \\
\hline$N$ & $22 \cdots \cdots \cdots \cdot 2$ & $N_{J}^{N_{22 \cdots 2}}$ & $\dot{u}_{\underbrace{122 \cdots 2}_{J}}$ & $\dot{u}_{\underbrace{222 \cdots 2}_{j}}$ & $\ldots .$. & $\dot{u}_{K} \underbrace{22 \cdots 2}_{J}$ \\
\hline
\end{tabular}

Fig. 1.1.1 $J$-way classification

Then we can evaluate the joint probability distribution that the sequences

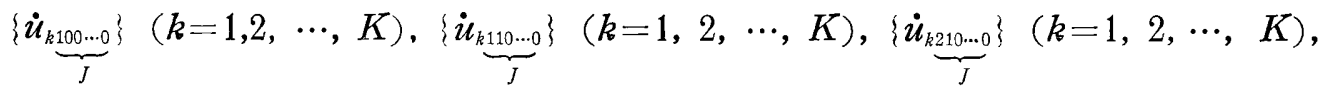
$\cdots,\{\underbrace{\left.\dot{u}_{k 22 \cdots 1}\right\}}_{J}(k=1,2, \cdots, K)$ are obtained for any assigned set of $\left\{b_{J}^{\left.b_{110 \cdots \cdots}\right\}}\right.$ $\{\underbrace{\left.b_{111.0}\right\}}_{J} \cdots, \quad\{\underbrace{b_{k 22 \cdots 1}}_{J}\}(k=1,2, \cdots, K)$, such that

$$
\begin{aligned}
& P\left\{\Pi\left(\dot{u}_{k 100 \cdots 0}=b_{k 100 \cdots 0}, \cdots, \dot{u}_{k 22 \cdots 1}=b_{k 22 \cdots 1}\right)\right\}
\end{aligned}
$$

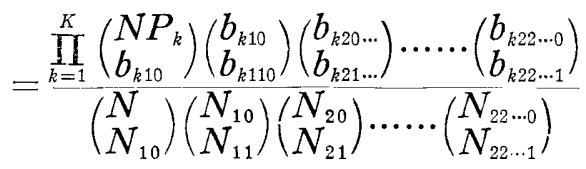

$$
\begin{aligned}
& =\frac{\left(N P_{1}\right) !\left(N P_{2}\right) ! \cdots \cdots\left(N P_{K}\right) !}{N !} .
\end{aligned}
$$

\subsection{Asymptotic distributions}

\subsubsection{Some transformations of variables}

So as to study the asymptotic distribution of the probability, such as (1.1.2), (1.1.3) and (1.1.4), let us consider the several transformations of 
the variables.

(1) Let us introduce a random variable, which is obtained by the prescribed random $J$-way classifition of $N\left(=2^{J}\right)$ elements of $\left\{v_{n}\right\} \quad(\boldsymbol{n}=1,2, \cdots$, $N)$, such that

$$
\dot{u}_{k_{n}} \stackrel{d}{=} \dot{u}_{\underbrace{}_{J 12 \cdots 1}},
$$

and which has the properties that we have $\dot{u}_{k n}=1$ for $\dot{v}_{\pi(n)}=\bar{v}_{k}$ and we have $\dot{u}_{k n}=0$ for $\dot{v}_{\pi(n)} \neq \bar{v}_{k}$. Then we get

$$
\dot{v}_{\pi(n)}=\sum_{k=1}^{K} \hat{v}_{k} \dot{u}_{k n}
$$

(2) We shall define a random variable

$$
\dot{V}_{k p} \stackrel{d}{=} \frac{1}{N} \sum_{n=1}^{N} x_{p n} \dot{u}_{k n}
$$

where we put $x_{p n}(p=1,2, \cdots, N ; n=1,2, \cdots, N)$ as a complete O.A. with size $N(=P+\Phi)$ which is composed of two sub-matrices

$$
D_{P}=x_{11} \cdots \cdots \cdots x_{1 N}
$$

and

$$
D_{\mathscr{Q}}=x_{P+1,1} \cdots x_{P+1, N}
$$

where $\Phi=N-P$ then we get

$$
x_{N, 1} \quad \cdots \quad x_{N N}
$$

$$
\begin{aligned}
\dot{V}_{p} & =\frac{1}{N} \sum_{n=1}^{N} x_{p n} \dot{v}_{\pi(n)} \\
& =\frac{1}{N} \sum_{k=1}^{K} \bar{v}_{k} \sum_{n=1}^{N} x_{p n} \dot{u}_{k n} \\
& =\sum_{k=1}^{K} \bar{v}_{k} V_{k p},
\end{aligned}
$$$$
(p=1,2, \cdots, N) .
$$

(3) Let us introduce a complete orthogonal array

$$
\begin{aligned}
& \left|\eta_{\mu \psi}^{(M)}\right|=\mid \begin{array}{l}
+++\cdots++ \\
+++\cdots--
\end{array} \\
& +++\cdots++ \\
& +++\cdots-- \\
& \text { - }, \quad \ldots \\
& +--\cdots-+ \\
& +-\cdots+-
\end{aligned}
$$


where $M=2^{J_{1}}(<N)$ is the size of the O.A. such as

$$
\begin{aligned}
& \eta_{\mu \psi}^{(2)}=\begin{array}{l}
+\leftarrow \\
+-{ }^{-}
\end{array} \quad\left(\begin{array}{l}
\mu=1,2 \\
\psi=1,2
\end{array}\right) \\
& ++++ \\
& n_{\mu \psi}^{(4)}=++- \\
& \div-+\quad, \quad\left(\begin{array}{l}
\mu=1,2,3,4 \\
\psi=1,2,3,4
\end{array}\right) \\
& +1
\end{aligned}
$$

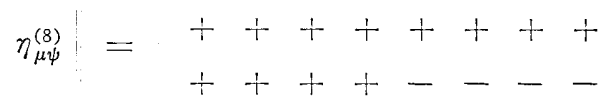

$$
\begin{aligned}
& ++--++-- \\
& \begin{array}{l}
++----++ \\
+-+-+-+-
\end{array} \quad, \quad\left(\begin{array}{l}
\mu=1,2, \cdots, 8 \\
\psi=1,2, \cdots, 8
\end{array}\right) \\
& +-+-++-+ \\
& +-1+-+ \\
& +--+-+
\end{aligned}
$$

and so on.

(4) In virtue of the matrix $\eta_{\mu \psi}^{(M)}$, we can define a $J_{1}$-way classification matrix

$$
y_{\mu n}^{(M)} \stackrel{d}{=} \frac{1}{M} \sum_{\psi=1}^{M} \eta_{\mu \psi}^{(M)} w_{\psi n}
$$

where $w_{\psi n}(\psi=1,2, \cdots, M ; n=1,2, \cdots, N)$ is the smallest complete suborthogonal array which contains

$$
\begin{aligned}
& \left\{x_{1 n}\right\}=x_{11} \cdots x_{1 N} \stackrel{d}{=} w_{11} \cdots w_{1 N} \\
& \left\{x_{p n}\right\}=x_{p 1} \cdots x_{p N} \stackrel{d}{=} w_{21} \cdots w_{2 N}
\end{aligned}
$$

and

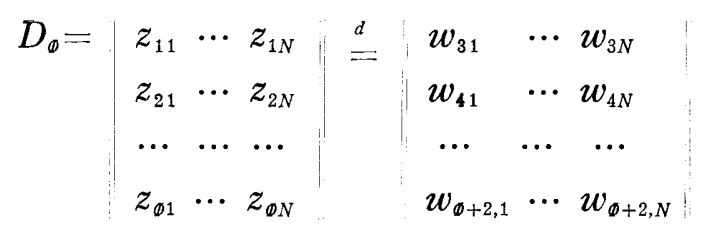

and where $\left\{x_{p n}\right\}(n=1,2, \cdots, N)$ for a particular $p(1 \leqq p \leqq P)$, is a row vector of $D_{P}$ corresponding to the statistic $\hat{\alpha}_{p}=\left(\alpha_{p}^{\prime}+\dot{V}_{p}^{\prime}+\frac{1}{N} \sum_{n=1}^{N} x_{p n} \dot{\varepsilon}_{n}\right)$ (refer to [12]). Then we get 
$(1.2 .15)$

$$
\begin{aligned}
& w_{\psi n}=x_{11} x_{12} \cdots x_{1 N} \\
& \begin{array}{llll}
x_{p 1} & x_{p 2} & \cdots & x_{p N}
\end{array} \\
& \begin{array}{llll}
z_{11} & z_{12} & \cdots & z_{1 N}
\end{array}
\end{aligned}
$$

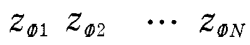

$$
\begin{aligned}
& \begin{array}{llll}
x_{i_{11}} & x_{i_{1}} & \cdots & x_{i_{1} N}
\end{array} \\
& \begin{array}{llll}
x_{i Q 1} & x_{i Q 3} & \cdots & x_{i Q 1}
\end{array} \\
& =\begin{array}{llll}
w_{11} & w_{12} & \cdots & w_{1 N}
\end{array} \\
& \begin{array}{llll}
w_{21} & w_{22} & \cdots & w_{2 N}
\end{array} \\
& \begin{array}{llll}
w_{M 1} & w_{M 2} & \cdots & w_{M N}
\end{array},
\end{aligned}
$$

where $i_{q}(q=1,2, \cdots, Q)$ are additional row numbers in $1 \leqq i_{q} \leqq P$ and $M=\Phi+2+Q$.

Then we get

$$
\dot{V}_{p}=\frac{1}{N} \sum_{n=1}^{N} x_{p n} \dot{v}_{(\pi n)}=\frac{1}{N} \sum_{n=1}^{N} w_{2 n} \dot{v}_{\pi(n)}=\dot{W}_{2},
$$

and in general

$$
\dot{W}_{\psi}=\frac{1}{N} \sum_{n=1}^{N} w_{\psi n} \dot{v}_{\pi(n)}
$$

$$
(\psi=1,2, \cdots, M)
$$

For example,

(4-1) for $J_{1}=1$, we have $M=2$ then

$$
\begin{aligned}
& \eta_{\mu \psi}^{(2)}=\begin{array}{l}
++ \\
+-
\end{array} \\
& \left(\begin{array}{l}
\mu=1,2 \\
\psi=1,2
\end{array}\right) \\
& \begin{array}{lllllllll} 
& 1 & 1 & \cdots & \cdots & 1 & 0 & 0 & \cdots
\end{array} \\
& y_{\mu n}^{(2)}=0 \underbrace{0 \cdots \cdots}_{\frac{N}{2}} 01 \frac{1 \cdots \cdots 1}{\frac{N}{2}} \text {, }
\end{aligned}
$$

(4-2) for $J_{1}=2$, we have $M=4$ then

$$
\eta_{\mu \psi}^{(4)}=\left|\begin{array}{c}
++++ \\
++-- \\
+-+- \\
+--+
\end{array}\right|, \quad\left(\begin{array}{l}
\mu=1,2,3,4 \\
\psi=1,2,3,4
\end{array}\right)
$$




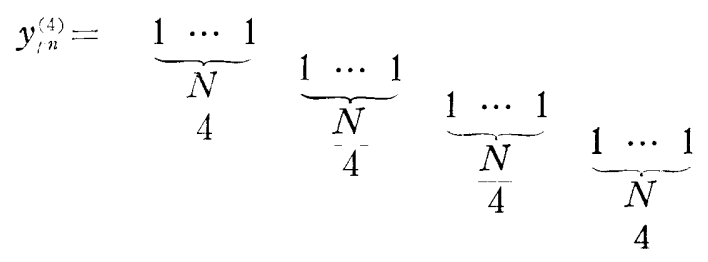

Consequently we get the relation between the random variables $\dot{u}_{k \mu}$ introduced in the random classification and the random variables $\dot{W}_{k \psi}$ introduced by the random pairing of $\dot{u}_{k n}(k=1,2, \cdots, K ; n=1,2, \cdots, N)$ and $w_{\psi n} \quad(\psi=1,2, \cdots, M ; n=1,2, \cdots, N)$, such that

$$
\begin{aligned}
\dot{\boldsymbol{u}}_{k \mu} & =\sum_{n=1}^{N} \boldsymbol{y}_{\mu n}^{(M)} \dot{\boldsymbol{u}}_{k n} \\
& =N \sum_{\psi=1}^{M} \eta_{\mu \psi}^{(M)} \dot{\boldsymbol{W}}_{k \psi},
\end{aligned}
$$

where

$$
\dot{W}_{k \psi}=\frac{1}{N} \sum_{n=1}^{N} w_{\psi n} \dot{u}_{k n} .
$$

Inversely, we have

$$
\dot{W}_{k \psi}=\frac{M}{N} \sum_{\mu=1}^{M} \eta_{\mu \psi}^{(M)}{ }^{-1} \dot{\boldsymbol{u}}_{k \mu}^{(M)},
$$

where $\left.\eta_{\mu \psi}^{(M)}\right|^{-1}$ is the inverse matrix of $\eta_{\mu \psi}^{(M)}$.

(5) Let us define a standardized variate

$$
\dot{t}_{k \psi}=\dot{W}_{k \psi} / \sqrt{\sigma^{2}\left(\dot{W}_{k \psi}\right)},
$$

where $\sigma^{2}\left(\dot{W}_{k \psi}\right)=$ aver $\left\{\dot{W}_{k \psi}^{2}\right\}-\operatorname{aver}^{2}\left\{\dot{W}_{k \psi}\right\}$. For the variate introduced by the prescribed random pairing $\quad \dot{W}_{k \psi}=\frac{1}{N} \sum_{n=1}^{N} w_{\psi n} \dot{u}_{k n}$, we have $\sigma^{2}\left(\dot{W}_{k \psi}\right)=\frac{1}{N^{2}}$ $\left\{N\left\langle 2_{k \psi}\right\rangle+N(N-1)\left\langle 1_{k \psi}^{2}\right\rangle-N^{2}\left\langle 1_{k \psi}\right\rangle^{2}\right\}$. Using Tukey's theorem [10] of random pairing which will be discussed in the next chapter of this paper, then we get

$$
\sigma^{2}\left(\dot{W}_{k \psi}\right)= \begin{cases}0 & , \text { for } \psi=1 \\ \frac{P_{k}\left(1-P_{k}\right)}{N-1} & , \text { for } \psi \geqq 2 .\end{cases}
$$

Hence, we have

$$
t_{k \psi}=\dot{W}_{k \psi} \sqrt{\frac{P_{k}\left(1-P_{k}\right)}{N-1}}, \text { for } \psi \geqq 2
$$


Using the result

$$
\dot{W}_{k 1}=\frac{1}{N} \sum_{n=1}^{N} w_{1 n} \dot{u}_{k n}=P_{k},
$$

we get

$$
\dot{u}_{k \mu}=\frac{N P_{k}}{M}\left(1+\dot{U}_{k \mu}^{(M)}\right)
$$

where

$$
\dot{U}_{k \mu} \stackrel{d}{=} \sum_{\psi=2}^{M} \eta_{\mu \psi}^{(M)} \dot{t}_{k \psi} \sqrt{\frac{1-P_{k}}{P_{k}^{(N-1)}}} .
$$

1.2.2. Asymptotic joint distribution function of statistics

In virtue of the results in section 1.1.2, for any assigned set of

$$
\begin{aligned}
& b_{k \mu}=b_{11} b_{12} \cdots b_{1 M} \\
& \begin{array}{llll}
\cdots & \ldots & \ldots & \ldots
\end{array} \\
& \begin{array}{llll}
b_{K 1} & b_{K 2} & \cdots & b_{K M}
\end{array} \text {, }
\end{aligned}
$$

we can evaluate the joint probability distribution function of the set of the statistics

is given by

$$
\begin{aligned}
& \dot{u}_{k \mu}=\dot{u}_{11} \dot{u}_{12} \cdots \dot{u}_{1 M} \\
& \begin{array}{llll}
\ldots & \ldots & \ldots & \ldots
\end{array} \\
& \dot{u}_{K 1} \dot{u}_{K 2} \cdots \dot{u}_{K M}
\end{aligned}
$$

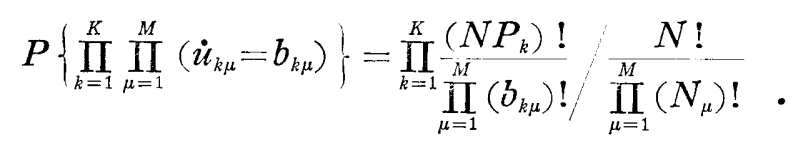

We can evaluate the limiting value of

$$
\begin{aligned}
\log P & \left\{\prod_{k=1}^{K} \prod_{\mu=1}^{M}\left(\dot{u}_{k \mu}=b_{k \mu}\right)\right\}=\sum_{k=1}^{K} \log \left(N P_{k}\right) !-\log N ! \\
& +\sum_{\mu=1}^{M} \log \left(N_{\mu}\right) !-\sum_{k=1}^{K} \sum_{\mu=1}^{M} \log b_{k \mu} !
\end{aligned}
$$

by virtue of the Stiring's formula. After some calculations, we get

$$
\begin{aligned}
\log P & \left\{\prod_{k=1}^{K} \prod_{\mu=1}^{M}\left(\dot{u}_{k \mu}=b_{k \mu}\right)\right\}=-\frac{1}{2}(K-1)(M-1) \log (2 \pi N) \\
& -\frac{1}{2}(M-1) \log \left(\prod_{k=1}^{K} P_{k}\right)+\frac{M}{2}(K-1) \log M \\
& -\sum_{k=1}^{K} \sum_{\mu=1}^{M}\left(b_{k \mu}+\frac{1}{2}\right) \log \left(1+B_{k \mu}\right)+\varepsilon_{1}^{\prime},
\end{aligned}
$$

where 


$$
\varepsilon_{1}^{\prime}=\frac{1}{12 N}\left\{\sum_{k=1}^{K} \frac{1}{P_{k}}-1+M^{2}-\sum_{\mu=1}^{M} \sum_{k=1}^{K} \frac{M}{P_{k}\left(1+B_{k \mu}\right)}+o\left(N^{-\frac{3}{2}}\right)\right\}
$$

and

$$
B_{k \mu} \stackrel{d}{=} \frac{M}{N P_{k}} b_{k \mu}-1 . \quad \text { Consequently, we get }
$$

where

$$
P\left\{\prod_{k=1}^{K} \prod_{\mu=1}^{M}\left(\dot{u}_{k \mu}=b_{k \mu}\right)\right\}=\frac{M^{M(K-1)} 2^{-12}}{(2 \pi N)^{\frac{(K-1)(M-1)}{2}}\left(\prod_{k=1}^{K} P_{k}\right)^{M-1} e^{-\sum_{\mu=1}^{M} \sum_{k=1}^{K} h_{k \mu}+\varepsilon_{1}^{\prime}}}
$$

$$
h_{k \mu}=\left(b_{k \mu} \div \frac{1}{2}\right) \log \left(1+B_{k \mu}\right) .
$$

Substituting the definition

$$
B_{k \mu}=\frac{M}{N P_{k}} b_{k \mu}-1
$$

we get

$$
h_{k \mu}=\left(\frac{N P_{k}}{M} B_{k \mu}+\frac{N P_{k}}{M}+\frac{1}{2}\right)\left\{B_{k \mu}-\frac{B_{k \mu}^{2}}{2}+\frac{B_{k \mu}^{3}}{3}-\cdots\right\} .
$$

Since

$$
\dot{U}_{k \mu}=\sqrt{\frac{1-P_{k}}{P_{k}(N-1)}} \sum_{\psi=2}^{M} \dot{t}_{k \psi} \eta_{\mu \psi}^{(M)},
$$

and

$$
\sum_{\mu=1}^{M} \dot{U}_{k \mu}=\sqrt{\frac{1-P_{k}}{P_{k}(N-1)} \sum_{\psi=2}^{M}} \dot{t}_{k \psi} \sum_{\mu=1}^{M} \eta_{\mu \psi}^{(M)}=0
$$

then we get

$$
\sum_{k=1}^{K} \sum_{\mu=1}^{M} h_{k \mu}=\sum_{k=1}^{K} \sum_{\mu=1}^{M} \frac{N P_{k}}{2} M B_{k \mu}^{2}+\varepsilon_{2}^{\prime}+o\left(N^{-2 / 3}\right),
$$

where

$$
\varepsilon_{2}^{\prime}=-\sum \sum \frac{N P_{k}}{6 M} B_{k \mu}^{3}+\sum \sum\left\{\frac{N P_{k}}{12 M} B_{k \mu}^{4}-B_{k \mu}^{2}\right\}
$$

Conseqnently, we get

\section{Theorem A}

For any assigned set of $b_{k \mu}(k=1,2, \cdots, K ; \mu=1,2, \cdots, M)$ the joint distribution function of the statistics $\dot{u}_{k \mu}(k=1,2, \cdots, K ; \mu=1,2, \cdots, M)$ is given by

$$
P\left\{\prod_{k=1}^{K} \prod_{\mu=1}^{M}\left(\dot{u}_{k \mu}=b_{k \mu}\right)\right\}=c e^{-\sum_{k} \sum_{\mu} \frac{N P_{k}}{2 M} B_{k \mu}{ }^{2}+\varepsilon_{1}^{\prime}+\varepsilon_{2}^{\prime}},
$$

where 


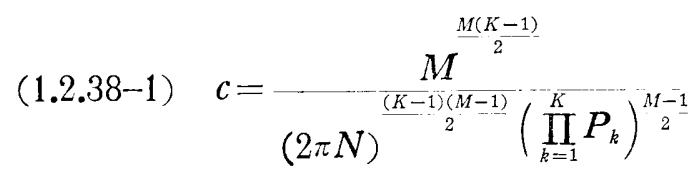

$(1.2 .38-2) \quad \varepsilon_{1}^{\prime}=-\sum_{k} \sum_{\mu} \frac{M}{12 N P_{k}\left(1+B_{k \mu}\right)}+\frac{1}{12 N}\left\{\sum_{k=1}^{K} \frac{1}{P_{k}}-1+M^{2}\right\}+o\left(N^{-3 / 2}\right)$

$(1.2 .38-3) \quad \varepsilon_{2}^{\prime}=-\frac{1}{6 M} \sum_{k} \sum_{\mu} N P_{k} B_{k \mu}^{3}+\sum_{k} \sum_{\mu}\left\{\frac{N P_{k}}{12 M} B_{k \mu}^{4}-\frac{1}{4} B_{k \mu}^{2}\right\}+o\left(N^{-3 / 2}\right)$

In the expression (1.2.38), we can put

$$
B_{k \mu}^{\prime} \stackrel{d}{=} \frac{N P_{k}}{M} B_{k \mu}=b_{k \mu}-\frac{N P_{k}}{M}
$$

then

$$
\sum_{\mu=1}^{M} B_{k \mu}^{\prime}=\sum_{\mu=1}^{M} b_{\mu k}-N P_{k}=0
$$

and

$$
\sum_{k=1}^{K} B_{k \mu}^{\prime}=\sum_{k=1}^{K} b_{k \mu}-\frac{N}{M} \sum_{k=1}^{K} P_{k}=0
$$

Consequently, we have

$$
\sum_{k=1}^{K} \sum_{\mu=1}^{M} \frac{N P^{k}}{M^{k}} B_{k \mu}^{2}=\sum_{k=1}^{K} \sum_{\mu=1}^{M} \frac{M}{N P_{k}}\left(B_{k \mu}^{\prime}\right)^{2}
$$

then

$$
P\left\{\prod_{k=1}^{K} \prod_{\mu=1}^{M}\left(\dot{u}_{k \mu}=b_{k \mu}\right)\right\}=c e^{-\frac{1}{2} \sum_{k=1}^{K} \sum_{\mu=1}^{M} \frac{M}{N P_{k}}\left(B_{k \mu}\right)^{2}+\varepsilon^{\prime \prime}},
$$

where

$$
c=\frac{M^{\frac{M}{2}(K-1)}}{(2 \pi N)^{\frac{1}{2}(K-1)(M-1)}\left(\prod_{k=1}^{K} P_{k}\right)^{\frac{1}{2}(M-1)}}
$$

and

$$
\varepsilon^{\prime \prime}=\varepsilon_{1}^{\prime}+\varepsilon_{2}^{\prime} \text {. }
$$

Since we get already in (1.2.16)

$$
\dot{u}_{k \mu}=\sum_{\psi=1}^{M}\left(\begin{array}{c}
1 \\
M
\end{array} \eta_{\mu \psi}^{(M)}\right)\left(N \dot{W}_{k \psi}\right)
$$

then we obtain

$$
\text { (1.2.46) } \quad \sum_{k=1}^{K} \sum_{\mu=1}^{M} \frac{M}{N P_{k}} B_{k \mu}^{\prime 2}=\sum_{k=1}^{K} \sum_{\psi=2}^{M} \frac{1}{N P_{k}}\left(N A_{k \psi}\right)^{2} \text {, }
$$

where $A_{k \psi}$ stands for the assigned set corresponding to the set of statistics $\dot{W}_{k \psi}$. Consequently, we get 


$$
P\left\{\prod_{k=1}^{K} \prod_{\psi=2}^{M}\left(N \dot{W}_{k \psi}=N A_{k \psi}\right)\right\}=c^{\prime} e^{-\frac{1}{2} \sum_{k=1}^{K} \sum_{\psi=2}^{M} \frac{1}{N P_{k}}\left(N A_{k \psi}\right)^{2}+\varepsilon^{\prime \prime}},
$$

where

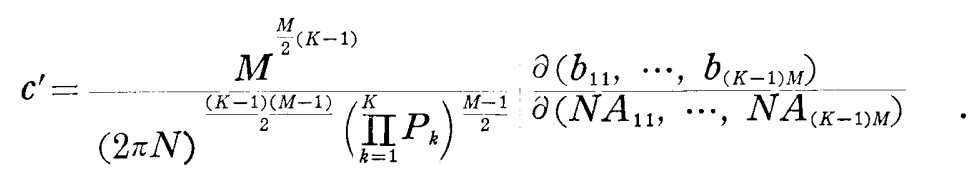

In the coefficient $c^{\prime}$, we put

$$
\begin{aligned}
& \frac{\partial\left(b_{11}, \cdots, b_{K-1, M}\right)}{\partial\left(N A_{11}, \cdots, N A_{(K-1) M}\right)} \\
& ={ }_{\| 1, \psi_{1}, \psi_{2}}{ } \partial_{2, \psi_{1}, \psi_{2}} \\
& \partial_{K-1, \psi_{1}, \psi_{2}}
\end{aligned}
$$

where

$$
\partial_{k, \psi_{1}, \psi_{2}} \stackrel{d}{=} \frac{\partial b_{k \psi_{1}}}{\partial\left(N A_{k \psi_{2}}\right)}
$$

and

Since

$$
\partial_{k, \psi_{1}, \psi_{2}}=\frac{\partial\left(b_{k 1}, \cdots, b_{k M}\right)}{\partial\left(N A_{k 1}, \cdots, N A_{k M}\right)}
$$

$$
\frac{\eta_{\mu \psi}^{(M)}}{M} \text { is an orthogonal matrix, then }
$$

$$
\left|\partial_{k, \psi_{1}, \psi_{2}}\right|=\mid \frac{1}{M^{\prime}} \eta_{\mu \psi}^{(M)}=\left(\frac{1}{\sqrt{M}}\right)^{M}
$$

and then we get

$$
\frac{\partial\left(b_{11}, \cdots, b_{K-1, M}\right)}{\partial\left(N A_{11}, \cdots, N A_{K-1, M}\right)}=\left(\frac{1}{\sqrt{M}}\right)^{M(K-1)}
$$

The coefficient (1.2.48) becomes

$$
c^{\prime}=\frac{1}{(2 \pi N)^{\frac{1}{2}(K-1)(M-1)}\left(\prod_{k=1}^{K} P_{k}\right)^{\frac{M-1}{2}}}
$$

then

$$
P\left\{\prod_{k=1}^{K} \prod_{\psi=2}^{M}\left(N \dot{W}_{k \psi}=N A_{k \psi}\right)\right\}=C^{\prime} e^{-\frac{1}{2} \sum_{k=1}^{K} \sum_{\psi=2}^{M} \frac{1}{N P_{k}}\left(N A_{k \psi}\right)^{2}+\varepsilon^{\prime \prime}},
$$

Here we shall introduce the variable

$$
A_{k \psi}^{\prime} \stackrel{d}{=} \sqrt{N} A_{k \psi}
$$

then the sum of $P\left\{\prod_{k=1}^{K} \prod_{\psi=2}^{M}\left(N \dot{W}_{k \psi}=N A_{k \psi}\right)\right\}$ 


$$
\begin{aligned}
& \sum \cdots \sum P\left\{\prod_{k=1}^{K} \prod_{\psi=2}^{M}\left(N \dot{W}_{k \psi}=N A_{k \psi}\right)\right\} \\
& \rightarrow \quad C^{\prime \prime} \int \cdots \int e^{-\frac{1}{2}} \sum_{k=1}^{K} \sum_{\psi=2}^{M} \frac{1}{P_{k}} A_{k \psi^{\prime}}^{2} d A_{12}^{\prime} \cdots d A_{k-1, M}^{\prime}, \quad N \rightarrow \infty
\end{aligned}
$$

where

$$
C^{\prime \prime}=-\frac{1}{(2 \pi)^{\frac{1}{2}(K-1)(M-1)}} \frac{\left.1 \prod_{k=1}^{K} P_{k}\right)^{\frac{1}{2}(M-1)}}{.}
$$

On the other hand, we have

$$
\sum_{k=1}^{K} P_{k}^{-1}\left(A_{\psi k}^{\prime}\right)^{2}=\boldsymbol{A}^{T} \boldsymbol{P} \boldsymbol{A}
$$

where $\boldsymbol{A}^{T}$ is the transposed matrix of $\boldsymbol{A}$ such that

$$
\boldsymbol{A}^{T}=A_{1 \psi}^{\prime}, A_{2 \psi}^{\prime}, \cdots, A_{K-1, \psi}^{\prime}
$$

and

$$
\boldsymbol{P}=\mid \begin{array}{cccc}
P_{1}^{-1}+P_{K}^{-1} & P_{K}^{-1} & \cdots & P_{K}^{-1} \\
P_{K}^{-1} & P_{2}^{-1}+P_{K}^{-1} & \cdots & P_{K}^{-1} \\
P_{K}^{-1} & & & \vdots \\
\vdots & \vdots & & \vdots \\
P_{K}^{-1} & P_{K}^{-1} & \cdots & P_{K-1}^{-1}+P_{K}^{-1}
\end{array} .
$$

Since we have the determinant of $\boldsymbol{P}$ such as

$$
\operatorname{det} \boldsymbol{P}=\left(\prod_{k=1}^{K} P_{k}^{-1}\right) / \sum_{j=1}^{K}\left(\frac{1}{\boldsymbol{P}_{j}^{-1}}\right)
$$

then the integral in the case that the order of $N^{-1 / 2}$ is negligible

$$
C^{\prime \prime} \int_{-\infty}^{\infty} \ldots \int_{-\infty}^{\infty} e^{-\frac{1}{2} \sum_{\psi=2}^{M} \sum_{k=1}^{K} \frac{1}{P_{k}} A_{k \psi^{2}}} d A_{12}^{\prime} \cdots d A_{K-1, M}^{\prime}=1 \text {. }
$$

Consequently in the same cases, we get the moment generating functon of $A_{\psi}=\sum_{k=1}^{K} \bar{v}_{k} A_{k \psi}$ corresponding the random variable in the expression (1.2.6), such that

$$
\begin{aligned}
q\left(A_{\psi}\right) & =E\left(e^{\theta \sum_{k=1}^{K} \bar{v}_{k} A_{k \psi}}\right) \\
& =e^{\frac{\theta^{2}}{2}\left(\frac{\mu_{2}}{N}\right)},
\end{aligned}
$$

where $\quad \mu_{2}=\sum_{k=1}^{K} \bar{v}_{k}^{2} P_{k}$. That is to say, the limiting distribution of $A_{\psi}=\sum_{k=1}^{K} \bar{v}_{k} A_{k \psi}$ is the normal distribution with mean 0 , variance $\mu_{2} / N$. Consequently, the joint limiting probability density function of $A_{2}, A_{3}, \cdots$, $A_{\oplus+2}$ is given by 


$$
\begin{gathered}
f\left(V_{\mu_{2}}^{N} A_{2}, \sqrt{\mu_{2}} A_{3}, \cdots, \sqrt{\mu_{2}^{N}} A_{\oplus+2}\right) \\
={ }_{(2 \pi)^{\frac{(\omega+2)}{2}}} e^{-\frac{1}{2} \sum_{\psi=2}^{\oplus+2}\left(\sqrt{\frac{N}{\mu_{2}}} A_{\psi}\right)^{2}}
\end{gathered}
$$

\section{Generalized symmetric means}

\section{Symmetric means}

First of all, let us consider the following results and notions by Tukey (refer to [9] and [10]) about the random pairings $\left\{x_{p 1}, v_{\pi(1)}\right\},\left\{x_{p 2}, v_{\pi(2)}\right\}$, $\cdots,\left\{x_{p N}, v_{\pi(N)}\right\}$ of two sets of $N$ numbers $x_{p 1}, x_{p 2}, \cdots, x_{p N}$ and $v_{\pi(1)}, v_{\pi(2)}, v_{\pi(3)}$, $\cdots, v_{\pi(N)}$, where $\pi(1), \pi(2), \cdots, \pi(N)$ is a permutation of the integers 1,2 , $\cdots, N$.

DEFINITION 2.1 ; (1) Let an $a$-th degree moment of multiplicative pairings be defined by

$$
\begin{aligned}
& <a_{p}>=\frac{1}{N} \sum X_{p n}^{a} \\
& X_{p n}=x_{p n} v_{\pi(n)}
\end{aligned}
$$

then we get the averaging over all pairings, which is denoted by the notation "aver"

$$
\begin{aligned}
\operatorname{aver}\left\{\left\langle a_{p}\right\rangle\right\} & \stackrel{d}{=} \\
& \left\langle\left\langle a_{p}\right\rangle\right\rangle \\
& =\left\langle a_{p}\right\rangle^{*}\langle a\rangle^{* *},
\end{aligned}
$$

where

$$
<a_{p}>^{*} \stackrel{d}{=} \frac{1}{N} \sum_{n=1}^{N} x_{p n}^{a}
$$

and

$$
<a>^{* *} \stackrel{d}{=} \frac{1}{N} \sum_{n=1}^{N} v_{\pi(n)}^{a}
$$

(2) Let us define $\mathbf{a}(a+b+\cdots+e)$-th degree symmetric mean

$$
\left\langle a_{p} b_{p} \cdots e_{p}>=\frac{1}{N^{(v)}} \sum_{1}^{N} \neq X_{p n_{1}}^{a} X_{p n_{2}}^{b} \cdots X_{p n_{\nu}}^{e}\right.
$$

of the multiplicative pairings $X_{p n}=x_{p n} v_{\pi(n)}$, where $\sum_{1}^{N} \neq$ means the distinct sum which is a sum taken over all subsequent subscripts, $\{n\}, n=1,2, \cdots, N$, but with subscripts kept different when they are indicated by different letters, such as

$$
\sum_{1}^{2} \neq x_{n} x_{n_{2}}=x_{1} x_{2}+x_{2} x_{1}
$$


and where $N^{(v)}$ is the number of terms in the summation, such that if we have different letters, $n_{1}, n_{2}, \cdots, n_{\nu}$,

$$
N^{(\nu)}=N \cdot(N-1) \cdots(N-\nu+1) .
$$

Then we have

$$
\begin{aligned}
\operatorname{aver} & \left.\left\langle a_{p} b_{p} \cdots e_{p}\right\rangle\right\} \stackrel{d}{=}\left\langle\left\langle a_{p} b_{p} \cdots e_{p}\right\rangle\right\rangle \\
= & \left\langle a_{p} b_{p} \cdots e_{p}\right\rangle^{*}\langle a b \cdots e\rangle^{* *},
\end{aligned}
$$

where

$$
<a_{p} b_{p} \cdots e_{p}>^{*} \stackrel{d}{=} \frac{1}{N^{(v)}} \sum_{1}^{N} x_{p n_{1}}^{+} x_{p n_{2}}^{b} \cdots x_{p n_{v}}^{e}
$$

and

$$
\langle a b \cdots e\rangle^{* *} \stackrel{d}{=} \frac{1}{N^{(v)}} \sum_{1}^{N}{ }_{n}^{+} v_{\pi\left(n_{1}\right)}^{a} \cdots v_{\pi\left(n_{v}\right)}^{e} \cdot
$$

2.2 Generalized Symmetric means: straightforward extensions of Tukey's symmetric means

\subsubsection{Column Generalized Symmetric means}

Let us define a generalized symmetric function of two way array

$$
\mid \begin{aligned}
& Z_{11} \cdot \cdot Z_{1 N} \\
& Z_{21} \cdot \cdot Z_{2 N}
\end{aligned}
$$

such that

$$
\text { (1) }\left(\begin{array}{c}
a b \cdots e \\
f g \cdots i
\end{array}\right)_{c}=\sum_{n}^{\ddagger} Z_{1 n_{1}}^{a} Z_{1 n_{2}}^{b} Z_{2 n_{1}}^{f} Z_{2 n_{2}}^{g} \cdots Z_{1 n_{m}}^{e} Z_{2 n_{m}}^{i}
$$

for $(a+b+\cdots+e+f+g+\cdots+i)$-th degree generalized symmetric function with respect to column, with which we shall use the abbreviated notation "c. g. s. f." here-after, where subscript "c" means the distinct sum over the column numbers.

For example, $(a+b+c+d)$-th degree c. g. s. f. is

$$
\text { (2) }\left(\begin{array}{ll}
a & b \\
c & d
\end{array}\right)_{c}=\sum_{n}^{\neq} Z_{1 n_{1}}^{a} Z_{1 n_{2}}^{b} Z_{2 n_{1}}^{c} Z_{2 n_{2}}^{d} \text {, }
$$

and $(a+b+c+d+e+f)$-th degree c. g. s. f. is

$$
\text { (1) }\left(\begin{array}{ccc}
a & b & c \\
d & e & f
\end{array}\right)_{c}=\sum_{n}^{\neq} Z_{1 n_{1}}^{a} Z_{1 n_{2}}^{b} Z_{1_{3}}^{c} Z_{1 n_{1}}^{d} Z_{2 n_{2}}^{e} Z_{2 n_{3}}^{f}
$$

and so on. In these abbreviated notations of c. g. s. f.'s, we shall use the preassigned subscripts “(1)", “(2)", etc, which represent the row number corresponding one in the population two-way array. 
Definition 2.2.: (1) For a two-way array

$$
Z_{\phi n}=\mid \begin{array}{cccc}
Z_{11} & Z_{12} & \cdots & Z_{1 N} \\
\cdot & \cdot & \cdots \\
\cdot & \cdot & \cdots \\
Z_{\sigma_{1}} & Z_{\sigma_{2}} & \cdots & Z_{\sigma_{N}}
\end{array} \quad,\left(\begin{array}{l}
\phi=1, \cdots, \\
n=1, \cdots, N
\end{array}\right)
$$

the column generalized symmetric function is defined by

(1) $a b \cdots$

(2) $f g \cdots k$

(3) $e m \cdots o \quad=\sum_{n}^{\ddagger} Z_{1 n 1}^{a} Z_{1 n 2}^{b} \cdots Z_{1 n v}^{e} Z_{2 n 1}^{f} Z_{2 n g}^{g} \cdots Z_{2 n v}^{k} \cdots$

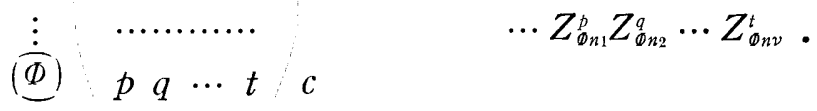

(2) As a straightforward extension of Tukey's symmetric means

$$
\langle a b \cdots e\rangle=\frac{[a b \cdots e]}{N^{(\nu)}}
$$

we can define a column generalized symmetric mean

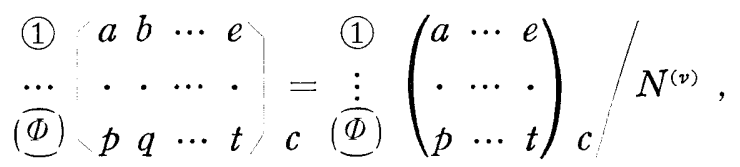

which will be abbreviated as c. g. s. m. here-after.

It must be noticed that a c. g. s. f.

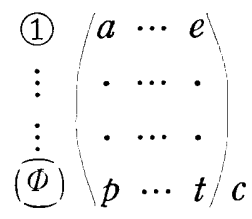

generates many other c. g. s. f.'s by the permutations with respect to columns, but c. g. s. f.'s permuted with respect to rows are not always equal to each other. That is to say, for example, we have

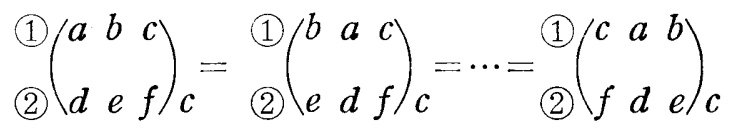

but it is not always held that

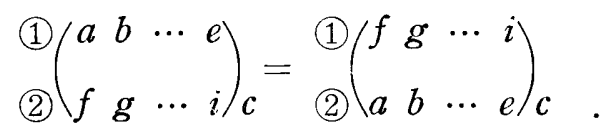

\subsubsection{Multiplications of c. g. s. f.'s}

The multiplication of these c. g. s. f.'s and c. g. s. m.'s is easily obtained as a slight extension of the multiplication of the brackets, 


$$
\begin{aligned}
(a)_{c}(b)_{c} & =\sum_{n} X_{n}^{a} \sum_{m} X_{m}^{b} \\
& =\sum X_{n}^{a+b}+\sum^{\ddagger} X_{m}^{a} X_{p}^{b} \\
& =(a+b)_{c}+(a b)_{c} .
\end{aligned}
$$

Thus

$$
\begin{aligned}
\left(\begin{array}{l}
a \\
0
\end{array}\right)_{c}\left(\begin{array}{l}
0 \\
b
\end{array}\right)_{c} & =\left(\sum Z_{f n}^{a}\right)_{c}\left(\sum Z_{g m}^{q}\right)_{c} \\
& =\sum Z_{f m}^{a} Z_{g m}^{b}+\sum^{\neq} Z_{f n} Z_{g m} \\
& =\left(\begin{array}{l}
a \\
b
\end{array}\right)_{c}+\left(\begin{array}{ll}
a & 0 \\
0 & b
\end{array}\right)_{c} .
\end{aligned}
$$

We can simplify these calculations as

$$
\left(\begin{array}{c}
a \\
-
\end{array}\right)_{c}(\vec{b})_{c}=\left(\begin{array}{l}
a \\
b
\end{array}\right)_{c}+\left(\begin{array}{l}
a-b \\
-b
\end{array}\right)_{c},
$$

where dashes " - " denote the zero entry

$$
\left(\begin{array}{c}
a-b \\
-b
\end{array}\right) \stackrel{d}{=}\left(\begin{array}{ll}
a & 0 \\
0 & b
\end{array}\right) \text {. }
$$

For a matrix with more than two rows, in a similar way, we have, for example
(1) $a b-$
(1) $--\cdots$
(1) $a b-$
(2) $-c d$
(2) $-\cdots$
$=$ (2) $-c d$
(1) $a b-$
(1) $a b-$
(3) $e f-$
(3) $e f-$
(2) $-c d$
(2) $-c d$
(4) $---1 /$
(3) ---
(4) $g h-/$
(3) $e f-$
(3) $e f-$
(4) $g h--/$
(1) $a b-$
(4) $g-h$ c
(4) $-g h / c$
+ (2) $-c d$
(1) $a b-$
(1) $a b-$
(3) $e f-$
(2) $-c d$
(2) $-c d$
(4) $h g-$ c
(3) $e f-$
(3) $e f-$
(1) $a b--$
(4) $h-g$ c
(4) $-h g$ c
+ (2) $-c d-$
(1) $a b--$
(1) $a b--$
(3) $e f--$
+ (2) $-c d-$
(2) $-c d-$
(4) $g--h / c$
(3) $e f--$
(3) $e f--$
(1) $a b \ldots$
(4) $-g-h$ :
(4) $--g h$ 。
+ (2) $-c d-$
(1) $a b--$
(1) $a b--$
(3) $e f--$
(2) $-c d-$
(2) $-c d-$
(4) $h--g \mid$
(3) $e f-$
(3) $e f--$
(1) $a b--$
(4) $-h-g$.
(4) $-h g$.
+ (2) $-c d--$
(4) $--g h / c$.

LEMMA 2.1: The product of two c. g. s. f.'s $\left(\varphi_{1} \times \nu_{1}\right)_{c}$ and $\left(\varphi_{2} \times \nu_{2}\right)_{c}$ by the 
following rule, such that, for $\nu_{1} \geq \nu_{2}$, is obtained

$$
\begin{aligned}
& \begin{array}{c:c|}
1 & \\
(2) & \varphi_{1} \times \nu_{1} \\
\vdots & \\
\hline\left(\varphi_{1}\right) & \\
\hline
\end{array} \\
& c_{c} \frac{\left(\varphi_{1}+1\right)}{\vdots} \varphi_{2} \times \nu_{2} \\
& =\Sigma^{\prime} \underset{\left(\begin{array}{l}
1 \\
\vdots \\
\left(\varphi_{1}+\varphi_{2}\right)
\end{array}\right.}{\left(\left(\varphi_{1}+\varphi_{2}\right) \times \nu_{1}\right)_{c}+\cdots+\sum_{\left(\varphi_{1}+\varphi_{2}\right)}^{\prime 1} \vdots}\left(\left(\varphi_{1}+\varphi_{2}\right) \times\left(\nu_{1}+1\right)\right)_{c}
\end{aligned}
$$

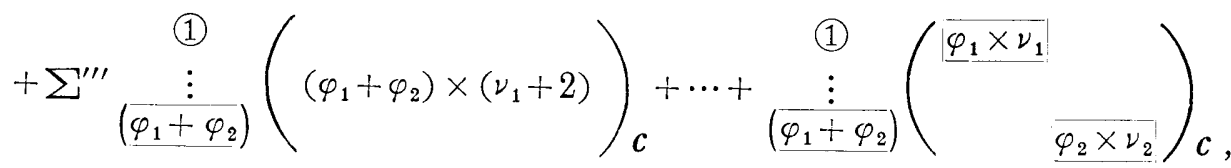

where the following remarks should be considered.

(i) $\Sigma$ ' stands for summation over all possible $\left(\varphi_{1}+\varphi_{2}\right)$ rows and $\nu_{1}$ columns g. s. f.'s ( )'s, which are generated by the combination of $\nu_{2}$ in the $\nu_{1}$ positions and permutations of $\nu_{2}$ numbers.

(ii) Second summation " $\Sigma$ " stands for the summation over all possible $\varphi_{1}+\varphi_{2}$ rows and $\nu_{1}+1$ columns g. s. f.'s which are generated by the rule that $\nu_{1}+1$ th column is chosen from the possible $\nu_{2}$ columns and $\nu_{2}-1$ possible columns are allocated in the possible $\nu_{1}$ column $\left(\begin{array}{l}\nu_{1} \\ \nu_{2}-1\end{array}\right)$ and $\nu_{2}-1$ columns permute each other all possible $\left(\nu_{2}-1\right)$ ! permutations.

(iii) The third summation " $\Sigma$ " " stands for the summation over all possible $\varphi_{1}+\varphi_{2}$ rows and $\nu_{1}+2$ columns g. $s$. f.'s which are generated by the rule that the possible $\nu_{1}+1$ th and $\nu_{2}+2$ th columns are chosen from the possible $\nu_{2}$ columns in $\left(\begin{array}{c}\nu_{2} \\ 2\end{array}\right)$ times and $\nu_{2}-2$ possible columns are allocated in the possible $\nu_{1}$ columns in $\left(\begin{array}{c}\nu_{1} \\ \nu_{2}-2\end{array}\right)$ times and $\nu_{2}-2$ columns permute each other all possible $\left(\nu_{2}-2\right)$ ! permutations, ana so on. (iv) The last term is obviously

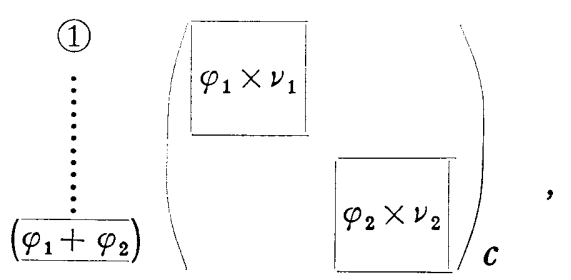

in which all $\nu_{2}$ columns are pushed out.

The proof of this lemma being virtually identical with that of (2.2.8) and (2.2.9), will be omitted. 


\subsubsection{Hooke's g. s. m.}

In virtue of $\mathrm{R}$. Hooke's paper [6], we can define a generalized symmetric function of a two-way array $Z_{\phi n}(\phi=1,2, \cdots, \Phi ; n=1,2, \cdots, N)$ such that

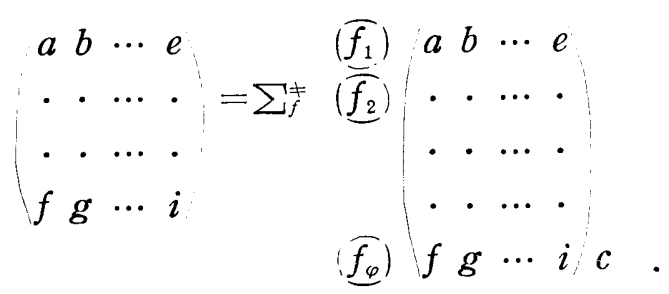

In this expression

$$
\frac{\left(\overline{f_{1}}\right)}{\underline{\dot{\vdots}}}\left(\underline{\underline{f_{\varphi}}}\right)()_{c}
$$

is the c. g. s. f. of a bisample

$$
\begin{array}{lll:l}
Z_{f_{1} n_{1}} & \cdots & Z_{f_{1} n_{\nu}} & \\
\cdot & \cdots & \cdot & \\
\cdot & \cdots & \cdot & \\
Z_{f \varphi n_{1}} & \cdots & Z_{f \varphi n_{\nu}} & ,(\varphi \leqq \Phi ; \nu \leqq N),
\end{array}
$$

which is sampled from the population two way array

Furthermore, we have

$$
\mid \begin{array}{ccc}
Z_{11} & \cdots & Z_{1 N} \\
\cdot & \cdots & \cdot \\
\cdot & \cdots & \cdot \\
Z_{\oplus 1} & \cdots & Z_{\oplus N}
\end{array}
$$

$$
\left(\begin{array}{cccc}
a & b & \cdots & e \\
\cdot & \cdot & \cdots & \cdot \\
f & g & \cdots & i
\end{array}\right)=\left(\begin{array}{cccc}
a & b & \cdots & e \\
\cdot & \cdot & \cdots & \cdot \\
\cdot & \cdot & \cdots & \cdot \\
f & g & \cdots & i
\end{array}\right)\left(\Phi^{(\varphi)} N^{(v)}\right.
$$

as g. s. f. and its g. s. m. by R. Hooke.

\subsubsection{Averaging over all pairings with respect to g. s. m. and c. g. s. m.'s}

Let us extend slightly from the prescribed Tukey's Theorem, i. e.

DEFinition 2.3: (1) Let us define the random pairings of a set of $N$ vectors $Z_{1}=\left(z_{11}, \cdots, z_{\oplus 1}\right), \cdots, Z_{N}=\left(z_{1 N}, \cdots, z_{\oplus N}\right)$ and a set of $N$ numbers $v_{1}$, $v_{2}, \cdots, v_{N}$ such that 


$$
\left(\begin{array}{llll}
Z_{1} & Z_{2} & \cdots & Z_{N} \\
v_{\pi(1)} & v_{\pi(2)} & \cdots & v_{\pi(N)}
\end{array}\right),
$$

where $\{\pi(n)\}(n=1,2, \cdots, N)$ is a permutation $\Pi$, which is chosen from the possible $N$ ! permutations of $N$ numbers with equal probability $1 / N$ !.

(2) Let us define the multiplicative random pairing

$$
Z_{\phi n}=z_{\phi n} v_{\pi(n)} \quad(\phi=1,2, \cdots, \Phi ; n=1,2, \cdots, N)
$$

and generalized symmetric mean (g. s. m.) in sense by Hooke

$$
\left(\begin{array}{ccc}
a & \cdots \cdots & e \\
\cdots & \cdots \cdots \cdots \\
f & \cdots \cdots & i
\end{array}\right)=\Sigma^{\mp} \Sigma^{ \pm} Z_{\phi_{1} n_{1}}^{a} \cdots \cdots \cdot Z_{\phi_{s} n_{\nu}}^{i} / \Phi^{(s)} N^{(v)} .
$$

LEMMA 2.2: The averaging over all possible g. s. m. is given by

(proof)

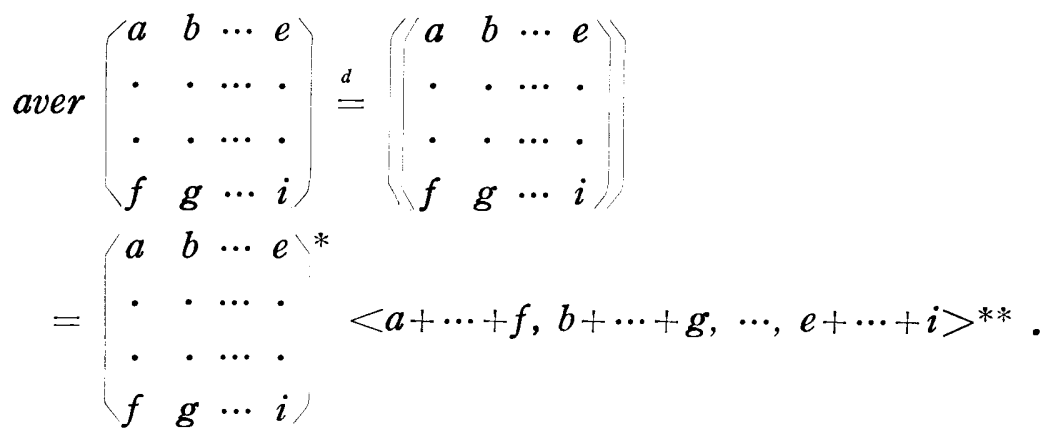

We have

$$
\begin{aligned}
& \operatorname{aver}\left(\begin{array}{cccc}
a & b & \cdots & e \\
\cdot & \cdot & \cdots & \cdot \\
\cdot & \cdot & \cdots & \cdot \\
f & g & \cdots & i
\end{array}\right)=\operatorname{aver} \frac{\sum^{\neq} \sum^{ \pm} Z_{\phi_{1} n_{1}}^{a} \cdots Z_{\phi_{s} \nu_{n}}^{i}}{N^{(v)} \Phi^{(s)}} \\
& \text { from the definition of g. s. m. } \\
& =\frac{\sum^{\ddagger} \sum_{N^{\ddagger}}^{\ddagger} z_{\phi_{1} n_{1}}^{a} \cdots z_{\phi_{S^{n}}}^{i}}{N^{(v)} \Phi^{(s)}} \text { aver }\left\{\dot{v}_{1}^{(a+\cdots+f)} \cdots \dot{v}_{n_{\nu}}^{(e+\cdots+i)}\right\} \\
& \text { from the definition of the random pairing } \\
& \left.=\left(\begin{array}{llll}
a & b & \cdots & e \\
\cdot & \cdot & \cdots & \cdot \\
\cdot & \cdot & \cdots & \cdot \\
f & g & \cdots & i
\end{array}\right)^{*}<a+\cdots+f, \cdots, e+\cdots+i\right\rangle^{* *} \text {. }
\end{aligned}
$$

COROLlARY OF LEMMA 4.2: The averaging over all $c . g . s . m$.

$$
\begin{aligned}
& \left(\underline{\phi_{1}}\right)\left(\begin{array}{llll}
a & b & \cdots & e
\end{array}\right. \\
& \text { - } \cdot \cdots \cdot=\sum_{n}^{\neq} Z_{\phi_{1} n_{1}}^{a} \cdots Z_{\phi_{s} n_{v}}^{i} / N^{(v)} \\
& \left.\left(\widehat{\left(\phi_{s}\right.}\right) \backslash f \quad g \quad \cdots i\right)_{c}
\end{aligned}
$$


is given by

$(2.2 .20)$

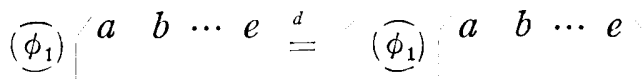

$$
\begin{aligned}
& \text { aver. }
\end{aligned}
$$

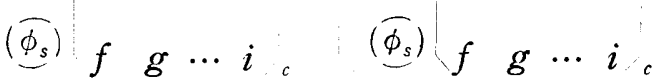

$$
\begin{aligned}
& \left(\widehat{\phi_{1}}\right) \quad a \quad b \quad \cdots \quad e^{* *} \\
& =\quad \cdot \quad \cdot \cdots \cdot\langle a+\cdots+f, \cdots, e+\cdots+i\rangle^{* *} \text {. } \\
& \left.\left(\widehat{\phi_{s}}\right) \backslash f \quad g \quad \cdots i\right)_{c}
\end{aligned}
$$

(proof)

In the similar manner to the proof of Lemma 2.2, we get

$$
\begin{aligned}
& \left(\underline{\phi}_{1}\right) a \cdots \cdots e
\end{aligned}
$$

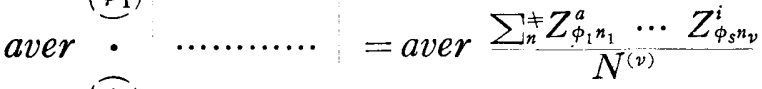

$$
\begin{aligned}
& \left(\widehat{\phi_{s}}\right) f \ldots \cdots, i, c \\
& =\frac{\sum_{n}^{\neq} z_{\phi_{1} n_{1}}^{a} \cdots z_{\phi_{s_{n}} n_{v}}^{i}}{N^{(v)}} \operatorname{aver}\left(v_{\pi^{\prime}\left(n_{1}\right)}^{a+\cdots+f} \cdots v_{\pi\left(n_{v}\right)}^{e+\cdots+i}\right) \\
& \left(\underline{\phi_{1}}\right)(a \cdots \cdots e)^{*}
\end{aligned}
$$

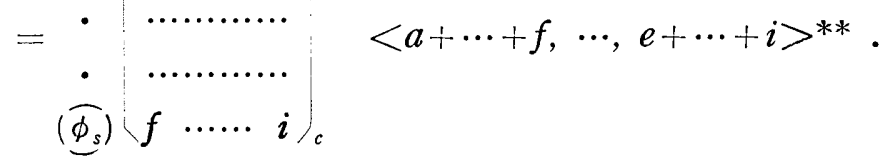

\subsection{Symmetric means of $v_{n}$ 's}

\subsubsection{Moments of $v_{n}$ 's}

Let us consider the symmetric means

$$
\langle a b \cdots e\rangle{ }^{* *}
$$

of a sequence

$$
v_{1}, v_{2}, \cdots, v_{N}=\beta_{1}, \cdots, \beta_{R}\left\|\begin{array}{ccc}
\xi_{11} & \cdots & \xi_{1 N} \\
\cdot & \cdots & \cdot \\
\cdot & \cdots & \\
\xi_{R 1} & \cdots & \xi_{R N}
\end{array}\right\| .
$$

In virtue of the tables of symmetric function by David and Kendall [4], which will be abbreviated as S. F. Tables hereafter, we can easily calculate these symmetric means, for example

$$
\begin{aligned}
& <1^{2}>^{* *}=\frac{1}{N^{(2)}}\left\{-(2)^{* *}+(1)^{* *^{2}}\right\}, \\
& <21>^{* *}=\frac{1}{N^{(2)}}\left\{-(3)^{* *}+(2)^{* *}(1)^{* *}\right\},
\end{aligned}
$$




$$
\begin{aligned}
<2>^{4 * *}=\frac{1}{N^{(4)}}\left\{-6(8)^{* *}+8(6)^{* *}(2)^{* *}+3(4)^{* * 2}\right. & \\
& \left.-6(4)^{* *}(2)^{* * 2}+(2)^{* * 4}\right\} .
\end{aligned}
$$

In these formulas, we need the following moments of the $v_{n}$ 's, for example

$$
\begin{aligned}
& (1)^{* *}=\sum_{1}^{N}\left(\sum_{r} \beta_{r} \xi_{r n}\right)=\sum_{r} \beta_{r} \sum_{1}^{N} \xi_{r n}=0 \\
& (2)^{* *}=\sum_{1}^{N}\left(\sum_{r} \beta_{r} \xi_{r n}\right)^{2}=\sum \beta_{r_{1}}^{2} \sum_{1}^{N} \xi_{r 1 n}^{2}+\sum \beta_{r_{2}} \beta_{r_{3}} \sum_{1}^{N} \xi_{r 2 n} \xi_{r 3 n} .
\end{aligned}
$$

Hence, we can define the moments of $v_{n}$ 's as follows,

$$
\mu_{1}^{\prime}(v) \stackrel{d}{=} \frac{(1)^{* *}}{N^{-}}=0, \quad \mu_{2}^{\prime}(v) \stackrel{d}{=} \frac{(2)^{* *}}{N}=\mu_{2}, \quad \mu_{3}^{\prime}(v) \stackrel{d}{=} \frac{(3)^{* *}}{N}=\mu_{3},
$$

and so on, where $\mu_{i}^{\prime}$ means $i$-th moment of $v_{n}$ 's about the origin and $\mu_{i}$ means ith moment of $v_{n}$ 's about the mean.

In the situation such that the matrix

$$
\left|\begin{array}{ccc}
\xi_{11} & \cdots & \xi_{1 N} \\
\cdot & \cdots & \cdot \\
\cdot & \cdots & \cdot \\
\xi_{R 1} & \cdots & \xi_{R N}
\end{array}\right|
$$

is a O.A. in which we have

$$
\begin{array}{ll}
\sum_{n=1}^{N} \xi_{r n} \xi_{s n} \begin{cases}=0 & r \neq s \\
=N & r=s\end{cases} \\
\sum_{n=1}^{N} \xi_{r n} \xi_{s n} \ldots \xi_{v n} \begin{cases}=0 & r, s, \cdots, v \text { are orthogonal } \\
=N & r, s, \cdots, v \text { are alias, }\end{cases}
\end{array}
$$

these moments of $v_{n}$ 's become the function only $\beta_{r}$ 's, such that for example $\mu_{4}=\sum \beta_{r}^{4}+\sum^{\neq} \beta_{r}^{2} \beta_{s}^{2}+\sum_{\text {alias }}^{\neq} \beta_{r} \beta_{s} \beta_{t} \beta_{u}$ where $\sum_{\text {allas }}^{\neq}$means the summuation over all combinations of rows having the alias relation.

\subsubsection{Symmetric means of $v_{n}$ 's}

We shall present the symmetric means of $v_{n}$ 's in table which shows the formulas such that

2 nd column $=$ sum of entries in the $3 \mathrm{rd}, 4$ th, $\cdots$, columns and each entry is equal to $N^{a-(b)}$ (Sum of terms of $\mu_{i}$ 's). In general, we have for the $r$ column symmetric functions

$$
\begin{gathered}
{[a \cdots e]=A_{1}(a) \cdots(e)+A_{2}(a+b)(c) \cdots(e)} \\
+\cdots+A_{r}(a+b+\cdots+e)
\end{gathered}
$$

where $A_{1}, \cdots, A_{r}$ are constants obtained by the combinatorial calculations. Hence, in the case that the $s_{1}^{*}$ unit entries are contained in the symmetric function of $v_{n}$ 's, we have $(1)^{* *}=0$ then 
Table 2-1 Symmetric means of $v_{n}$ 's

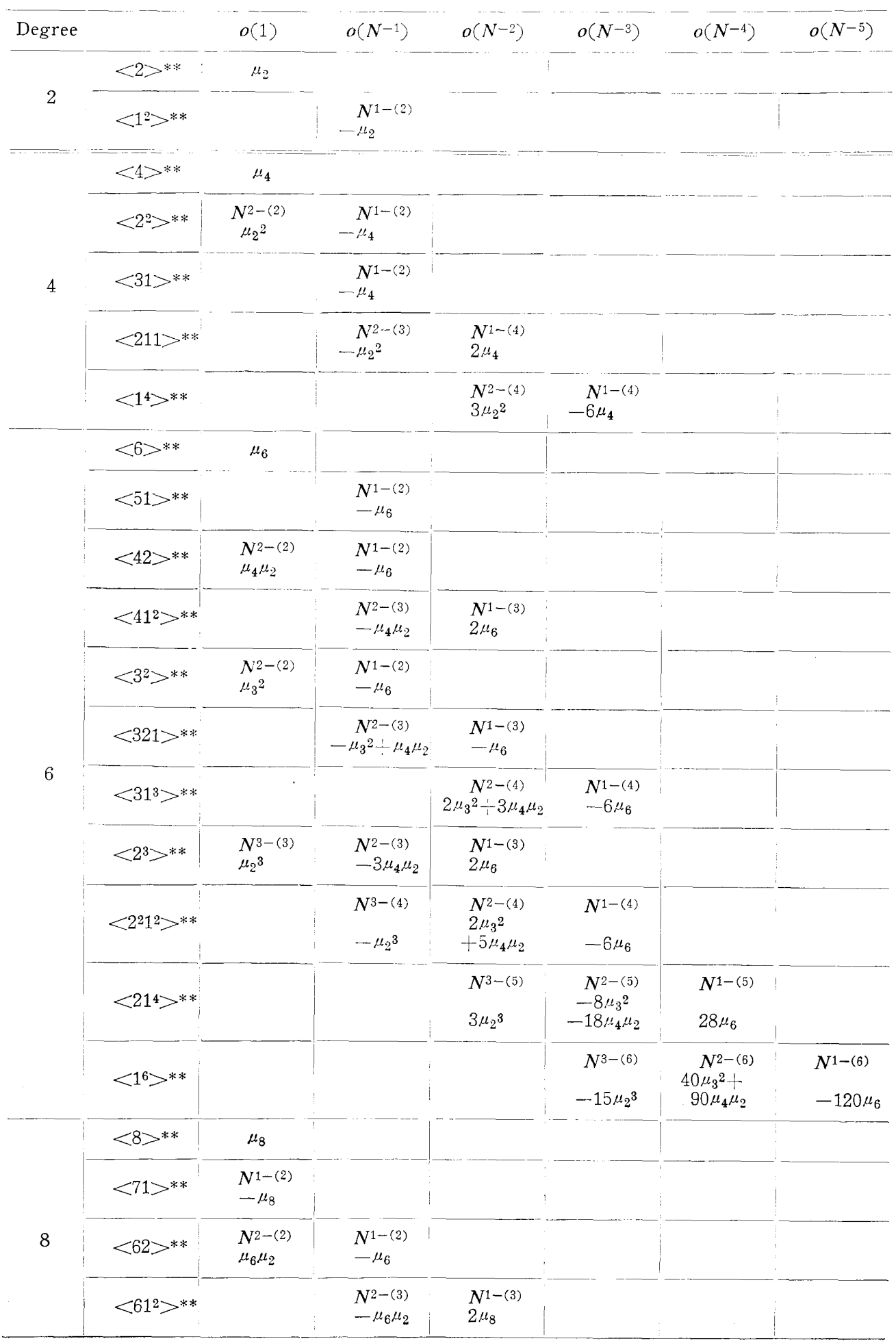


Table 2-1 Continue

\begin{tabular}{|c|c|c|c|c|c|c|c|c|c|}
\hline Degree & & $o(1)$ & $o(N-1)$ & $o\left(N^{-2}\right)$ & $o\left(N^{-3}\right)$ & $o\left(N^{-4}\right)$ & $o\left(N^{-5}\right)$ & $o\left(N^{-6}\right)$ & $o\left(N^{-7}\right)$ \\
\hline \multirow{15}{*}{8} & $<53>^{* *}$ & $\begin{array}{l}N^{2-(2)} \\
\mu_{5} \mu_{3}\end{array}$ & $\begin{array}{l}N^{1-(2)} \\
-\mu_{8}\end{array}$ & & & & & & \\
\hline & $<521>* *$ & & $\begin{array}{l}N^{2-(3)} \\
-\mu_{5} \mu_{3} \\
-\mu_{6} \mu_{2}\end{array}$ & $\begin{array}{l}N^{1-(3)} \\
2 \mu_{8}\end{array}$ & & & & & \\
\hline & $<51^{3}>* *$ & & & $\begin{array}{l}N^{2-(4)} \\
2 \mu_{5} \mu_{3} \\
3 \mu_{6} \mu_{2}\end{array}$ & $\begin{array}{l}\mathbf{N}^{1-(4)} \\
-6 \mu_{8}\end{array}$ & & & & \\
\hline & $\left\langle 4^{2}\right\rangle * *$ & $\begin{array}{l}N^{2-(2)} \\
\mu_{4}^{2}\end{array}$ & $\begin{array}{l}N^{1-(2)} \\
-\mu_{8}\end{array}$ & & & & & & \\
\hline & $<431>* *$ & & $\begin{array}{l}N^{2-(3)} \\
-\mu_{4}^{2} \\
-\mu_{5} \mu_{3}\end{array}$ & $\begin{array}{l}N^{1-(3)} \\
2 \mu_{8}\end{array}$ & & & & & \\
\hline & $<42^{2}>{ }^{*}$ & $\begin{array}{l}N^{3-(3)} \\
\mu_{4} \mu_{2} 2\end{array}$ & $\begin{array}{l}N^{2-(3)} \\
-\mu_{4}^{2} \\
-2 \mu_{6} \mu_{2}\end{array}$ & $\begin{array}{l}N^{1-(3)} \\
2 \mu_{8}\end{array}$ & & & & & . \\
\hline & $<421^{2}>* *$ & & $\begin{array}{l}N^{3-(4)} \\
-\mu_{4} \mu_{2}{ }^{2}\end{array}$ & $\begin{array}{l}N^{2-(4)} \\
2 \mu_{4}^{2} \\
2 \mu_{5} \mu_{3} \\
3 \mu_{6} \mu_{2}\end{array}$ & $\begin{array}{l}N^{1-(4)} \\
-6 \mu_{8}\end{array}$ & & & & \\
\hline & $<41^{4}>* *$ & & & $\begin{array}{l}N^{3-(5)} \\
3 \mu_{4} \mu_{2}^{2}\end{array}$ & $\begin{array}{l}N^{2-(5)} \\
-6 \mu_{4}^{2} \\
-8 \mu_{5} \mu_{3} \\
-12 \mu_{6} \mu_{2}\end{array}$ & $\begin{array}{l}N^{1-(5)} \\
24 \mu_{8}\end{array}$ & & . & i \\
\hline & $<322>* *$ & $\begin{array}{l}N^{3}-(3) \\
\mu_{3} 2 \mu_{3}\end{array}$ & $\begin{array}{l}N^{2-(3)} \\
-2 \mu_{5} \mu_{3} \\
-\mu_{6} \mu_{2}\end{array}$ & $\begin{array}{l}N^{1-(3)} \\
2 \mu_{8}\end{array}$ & & & & & \\
\hline & $<3^{2} 1^{2}>* *$ & & $\begin{array}{l}N^{3-(4)} \\
-\mu_{3}{ }^{2} \mu_{2}\end{array}$ & $\begin{array}{l}N^{2-(4)} \\
2 \mu_{4}^{2} \\
4 \mu_{5} \mu_{3} \\
\mu_{6} \mu_{2}\end{array}$ & $\begin{array}{l}N^{1-(4)} \\
-6 \mu_{8}\end{array}$ & & & & \\
\hline & $<32^{2} 1>^{* *}$ & & $\begin{array}{l}N^{3-(4)} \\
-2 \mu_{3}{ }^{2} \mu_{2} \\
-\mu_{4} \mu_{2}{ }^{2}\end{array}$ & $\begin{array}{l}N^{2-(4)} \\
\mu_{4}^{2} \\
4 \mu_{5} \mu_{3} \\
4 \mu_{6} \mu_{2}\end{array}$ & $\begin{array}{l}N^{1-(4)} \\
-6 \mu_{8}\end{array}$ & & & & \\
\hline & $<321^{3}>* *$ & & & $\begin{array}{l}N^{3-(5)} \\
5 \mu_{3} 2 \mu \\
3 \mu_{4} \mu_{2} 2\end{array}$ & $\begin{array}{l}N^{2-(5)} \\
-6 \mu_{4}{ }^{2} \\
-14 \mu_{5} \mu_{3} \\
-12 \mu_{6} \mu_{2}\end{array}$ & $\begin{array}{l}N^{1-(5)} \\
24 \mu_{8}\end{array}$ & & & \\
\hline & $<31^{5}>* *$ & & & & $\begin{array}{l}N^{3-(6)} \\
-20 \mu_{3}{ }^{2} \mu_{2} \\
-15 \mu_{4} \mu_{2}{ }^{2} \\
\end{array}$ & $\begin{array}{l}N^{2-(6)} \\
30 \mu_{4}^{2} \\
64 \mu_{5} \mu_{3} \\
60 \mu_{6} \mu_{2}\end{array}$ & $\begin{array}{l}N^{1-(6)} \\
-120 \mu_{8}\end{array}$ & & \\
\hline & $\left.<2^{4}\right\rangle^{* *}$ & $\begin{array}{l}N^{4-(4)} \\
\mu_{2}^{4}\end{array}$ & $\begin{array}{l}N^{3-(4)} \\
-6 \mu_{4} \mu_{2} 2\end{array}$ & $\begin{array}{l}N^{2-(4)} \\
3 \mu_{4}^{2} \\
8 \mu_{6} \mu_{2}\end{array}$ & $\begin{array}{l}N^{1-(4)} \\
-6 \mu_{8}\end{array}$ & & & & \\
\hline & $<2^{3} 1^{2}>* *$ & & $\begin{array}{l}N^{4-(5)} \\
-\mu_{2}\end{array}$ & $\begin{array}{l}N^{3-(5)} \\
6 \mu_{3}^{2} \mu_{2} \\
9 \mu_{4} \mu_{2} 2\end{array}$ & $\begin{array}{l}N^{2-(5)} \\
-6 \mu_{4}^{2} \\
-12 \mu_{5} \mu_{3} \\
-20 \mu_{6} \mu_{2}\end{array}$ & $\begin{array}{l}N^{1-(5)} \\
24 \mu_{3}\end{array}$ & & & \\
\hline
\end{tabular}




\begin{tabular}{|c|c|c|c|c|c|c|c|c|c|}
\hline Degree & & $o(1)$ & $o\left(N^{-1}\right)$ & $o\left(N^{-2}\right)$ & $o\left(N^{-3}\right)$ & $o\left(N^{-4}\right)$ & $o\left(N^{-5}\right)$ & $o\left(N^{-6}\right)$ & $o\left(N^{-7}\right)$ \\
\hline \multirow{3}{*}{8} & $<2^{2} 1^{4}>{ }^{* *}$ & & & $\begin{array}{l}N^{4-(6)} \\
3 \mu_{2}^{4}\end{array}$ & $\begin{array}{l}N^{3-(6)} \\
-28 \mu_{3}^{2} \mu_{2} \\
-33 \mu_{4} \mu_{2}{ }^{2}\end{array}$ & $\begin{array}{l}N^{2-(6)} \\
30 \mu_{4}^{2} \\
64 \mu_{5} \mu_{3} \\
84 \mu_{6} \mu_{2}\end{array}$ & $\begin{array}{l}N^{1-(6)} \\
-120 \mu_{8}\end{array}$ & & \\
\hline & $<21^{6}>* *$ & & & & $\begin{array}{l}N^{4-(7)} \\
15 \mu_{2}{ }^{4}\end{array}$ & $\begin{array}{l}N^{3-(7)} \\
160 \mu_{3}{ }^{2} \mu_{2} \\
180 \mu_{4} \mu_{2}{ }^{2}\end{array}$ & $\begin{array}{c}N^{2-(7)} \\
-180 \mu_{4}^{2} \\
-384 \mu_{5} \mu_{3} \\
-480 \mu_{6} \mu_{2}\end{array}$ & $\begin{array}{l}N^{1-(7)} \\
720 \mu_{8}\end{array}$ & \\
\hline & $<1^{8}>^{* *}$ & & & & & $\begin{array}{l}N^{4-(8)} \\
105 \mu_{2}{ }^{4}\end{array}$ & $\begin{array}{c}N^{3-(8)} \\
-1120 \mu_{3}{ }^{2} \mu_{2} \\
-1260 \mu_{4} \mu_{2} 2\end{array}$ & $\begin{array}{l}N^{2-(8)} \\
1260 \mu_{4}{ }^{2} \\
2688 \mu_{5} \mu_{3} \\
3360 \mu_{6} \mu_{2}\end{array}$ & $\begin{array}{l}N^{1-(8)} \\
-5040 \mu_{8}\end{array}$ \\
\hline
\end{tabular}

$$
\langle a \cdots e \underbrace{1 \cdots 1}_{s 1^{*}}\rangle^{* *}=o\left(N^{-\frac{s_{1}^{*}}{2}}\right) .
$$

\subsection{Two way array finite population}

\subsubsection{Symmetric means of a row in O.A.}

In virtue of O.A., we have

$$
\begin{aligned}
\left(a_{p}\right) *=\sum_{n=1}^{N} x_{p n}^{a} & =0 & & \text { if “ } a \text { " is odd } \\
& =N & & \text { if “ } a \text { " is even, }
\end{aligned}
$$

then following the other sort of symmetric functions can be easily obtained in refering to S.F. Tables.

Using these results, we get the symmetric means of one row in O.A.

$$
\begin{array}{ll}
\langle a b c \cdots e\rangle^{*}=1 & \text { if all } a, b, c, \cdots, e \text { are even } \\
\langle a b c \cdots e\rangle^{*}=0 & \begin{array}{l}
\text { if the order of the symmetric mean, } \\
\\
(a+b+\cdots+e), \text { is odd }
\end{array}
\end{array}
$$

$$
<a b c \cdots e\rangle^{*}=\frac{-1}{N-1}, \quad 2 \text { characters are odd }
$$

$$
\begin{aligned}
& =\frac{(-1)(-3)}{(N-1)(N-3)}, \\
& =\frac{(-1)(-3)(-5)}{(N-1)(N-3)(N-5)}, \\
& =\frac{(-1)(-3)(-5)(-7)}{(N-1)(N-3)(N-5)(N-7)}, 8 \quad \prime \prime \prime \text {. }
\end{aligned}
$$


In general, we have for the s. f.'s

(2.4.5) $\quad[a b \cdots e]=A_{1}(a)(b) \cdots(e)+A_{2}(a+b)(c) \cdots(e)+\cdots+A_{r}(a+b+\cdots+e)$. Since odd number has no partition which is constructed by all even entries, then the odd degree s. f. and s. m. denoted as [(odd) $]^{*}$ and $\langle\text { (odd) }\rangle^{*}$, are (2.4.6) $[(\text { odd })]^{*}=0$

and

$$
<\text { (odd) }\rangle^{*}=0 \text {. }
$$

Since the even degree s. f. contains even odd entries, we have

$$
[a b \cdots e \alpha \beta \cdots \varepsilon]=A_{1}(a)(b) \cdots(\alpha+\beta) \cdots(\delta+\varepsilon)+\cdots+A_{i}(a+\cdots+\varepsilon),
$$

where $a, b, \cdots, e$ stand for even entries and $\alpha, \beta, \cdots, \varepsilon$ stand for odd entries. In virtue of formula (2.4.1), we have

$$
[a \cdots e \alpha \cdots \varepsilon]^{*}=o\left(N^{s^{*}+\frac{s 2^{*}}{2}}\right),
$$

where $s_{1}{ }^{*}, s_{2}{ }^{*}$ stand for the sum of even and odd entries, respectively.

Consequently we get

$$
\langle a b \cdots e \alpha \cdots \varepsilon\rangle^{*}=o\left(N^{-\frac{s_{2}^{*}}{2}}\right) .
$$

Furthermore, since the $2 K$-th degree s. f. has the highest $s_{1}{ }^{*}$ when the all entries are $2, s_{1}^{*}=K$, in the case that the number of entries $k$ is larger than $K$, we have $s_{2}^{*}$ unit entries,

$$
s_{2}^{*}=k-K,
$$

then

$$
\overbrace{22 \cdots 2}^{s_{1 *}^{*}} \overbrace{1 \cdots 1}^{s 2^{*}}\rangle^{*}=o\left(N^{-\frac{k-K}{2}}\right) .
$$

\subsubsection{Relations between rows of O.A.}

So as to calculate the g. s. f.'s of $z_{\phi n}(\phi=1,2, \cdots, \Phi ; n=1,2, \cdots, N)$ we shall average over all c. g. s. f.'s of possible bisamples,

$$
\begin{array}{|ccc|}
z_{f 11} & \cdots & z_{f_{1} N} \\
\cdot & \cdots & \cdot \\
\cdot & \cdots & \cdot \\
z_{f \varphi 1} & \cdots & z_{f \varphi N}
\end{array} .
$$

Let us consider the c. g. s. f.'s of orthogonal array with two levels. In these O.A., we have alias relations (refer to Box \& Hunter [2] [3] and Shimada [8]) with respect to particular three or more rows, such that

$\begin{array}{ccc}\text { row No } & & \text { relation } \\ 1 & = & \text { (1) } \\ 2 & = & (2 \\ 3 & = & (3 \\ 4 & = & \text { (12) }\end{array}$




$\begin{array}{lll}5 & = & (13) \\ 6 & = & (123) \\ 7 & =\end{array}$

In this table, the relation column means that for example the individuals in the (12) or (13) or (23) or (123) column are equal to the product of the individuals in the (1) and (2), or (1) and (3) or (2) and (3) columns which are components of the number of relations (12) or (13) or (3) or (123). That is to say, $z_{1 n} z_{2 n}=z_{4 n}, z_{2 n} z_{3 n}=z_{6 n}$, and $z_{1 n} z_{2 n} z_{3 n}=z_{7 n}$, and so on. These relations are the well known "alias" relations.

In our calculations of c. g. s. f.'s of O.A., we shall represent the c. g. s. f.'s with relation numbers in places of row number so as to distinct the alias relation in these rows to orthogonal relation shall denote "alias" or "orth" at the bottom of c. g. s. f.'s and c. g. s. m.'s. With respect to the relation numbers it must be noticed that the third relation between the several rows sampled from the O. A. exists in addition to the above alias and orthogonal relations. That is to say, the case that the alias relation exists in a part of all these sampled rows, such that for example

$$
\left.\begin{array}{l}
\text { (1), (2), (12), (3)) } \\
\text { (1), (2), (12), (3), (123)) }
\end{array}\right\} \rightarrow \text { partially alias }
$$

and so on, in addition to the relations such that, for example

(1), (2), (3), (4), - $\rightarrow$ orthogonal

(1), (2), (3), (123), $\longrightarrow$ alias

Furthermore, for the orthogonal relation, we have (1), (2), $\cdots,(\phi)$ equivalent relation in the permuted row number (1), (ब) $, \cdots,(5)$ and for the alias relation (1), (2), $\cdots,(\bar{\phi})$, and $(12 \cdots \bar{\phi})$ we have equivalent relation in the permuted relation number, (5), (3), $\cdots,(12 \cdots),, \cdots,(2)$. On the other hand, in the third relation, we have not equivalent relation in the permuted sequence of the row numbers.

\subsubsection{Diagonal c. g. s. f.'s of O.A.}

In virtue of orthogonality of O.A., we have

$$
\left(\begin{array}{l}
1 \\
1
\end{array}\right)_{c}=0 \text {, }
$$

and in general

$$
\left(\begin{array}{c}
1 \\
1 \\
\vdots \\
1
\end{array}\right)_{c r t h}=0
$$$$
\left(\begin{array}{c}
1 \\
1 \\
\vdots \\
1
\end{array}\right)_{c l i a s}=N
$$ 
where $\left(\begin{array}{c}1 \\ 1 \\ \vdots \\ 1\end{array}\right)_{\text {orth }}$ represents the c. g. s. f. having rows in a orthogonal relation such that for example (1), (2), and (3), and $\left(\begin{array}{c}1 \\ \vdots \\ \vdots \\ 1\end{array}\right)_{c}$ represents the c. g. s. f. having the rows in an alias relations, such that for example (1), (2) and (12).

Since the orthogonal or alias relations are held in the permutation of rows, the column g. s. f.'s, which are generated by the permutations of rows are equal to each other in the case the alias relation and orthogonal relation are held in the sampled rows.

On the other hand, for a rows of $z_{p n}$ we can also denote

$$
\begin{aligned}
& \text { (1) }(1)_{c}(1)(1)_{C}=\frac{(1)}{(1)}\left(\begin{array}{l}
1 \\
1
\end{array}\right)_{C}+\frac{1}{(1}(1-1)_{C} \\
& \left(\begin{array}{l}
1 \\
(1)
\end{array}\left(\begin{array}{l}
1 \\
1
\end{array}\right)_{C}(1)(1)=\frac{1}{1}\left(\begin{array}{l}
1 \\
1 \\
1
\end{array}\right)_{C}+\frac{(1)}{(1)}\left(\begin{array}{c}
1- \\
1-
\end{array}\right)_{C}\right.
\end{aligned}
$$

and so on. In general, all s. f.'s in the S.F. Tables by David and Kendall can be denoted as the c. g. s. f.'s partitioned into several rows so as to partition into unit entries.

Similarly, in the case the alias and orthogonal relations are in our $\mathrm{O}$. A., we have

and

$$
\text { (1) }(1)=\left(\begin{array}{l}
1 \\
1
\end{array}\right)+\left(\begin{array}{l}
1- \\
-1
\end{array}\right)
$$

$$
\text { (1) } \underset{\text { alias }}{(1)}(1)=\left(\begin{array}{l}
1 \\
1 \\
1
\end{array}\right)+3\left(\begin{array}{c}
1- \\
1-1 \\
\text { alias }
\end{array}\right)+\left(\begin{array}{c}
1-- \\
-1- \\
-\frac{1}{\text { alias }}
\end{array}\right)
$$

and so on, where ( )'s are the abbreviated notation of c. g. s. f.'s with no subscript and no pre-assigned row number so as to represent the analogy of s. f.'s for a row.

We can tabulate these relations in the style of the S.F. Tables. such that for example

\begin{tabular}{|l|lcc|}
\hline & $\left(\begin{array}{l}1 \\
1 \\
1\end{array}\right)$ & $\left(\begin{array}{c}1- \\
1 \\
-1\end{array}\right)$ & $\left(\begin{array}{c}1-- \\
-1-\end{array}\right)$ \\
\hline$\left(\begin{array}{l}1 \\
1 \\
1\end{array}\right)$ & $\mathbf{1}$ & -1 & 2 \\
$\left(\begin{array}{l}1 \\
1\end{array}\right)(1)$ & 1 & $\mathbf{1}$ & -3 \\
$(1)(1)(1)$ & 1 & 3 & $\mathbf{1}$ \\
\hline
\end{tabular}


and so on.

These tables are, of course, equivalent to the S.F. Tables except the notation of s. f.'s in the 1st column and 1st row, which are partitioned to different rows.

In our third condition, in which the alias relation exists in a part of the sampled rows, we have also the formulas (2.4.17) etc.

Consequently, we can use these tables in the same manner to converge the product-sums, such as $\left(\begin{array}{l}1 \\ 1 \\ 1 \\ 1\end{array}\right),\left(\begin{array}{l}1 \\ 1\end{array}\right)$ and so on, into terms of c. g. s. f.'s or vice versa.

Then we get easily

$$
\begin{aligned}
& \left(\begin{array}{c}
1-1 \\
-1
\end{array}\right)_{C}=0 \text {, } \\
& \left(\begin{array}{c}
1-\overline{-} \\
-1-\underset{\text { orth }}{1}
\end{array}\right)_{C}=0, \quad\left(\begin{array}{c}
1-\overline{-} \\
-\frac{1-}{\text { alias }}
\end{array}\right)_{C}=2 N, \\
& \left(\begin{array}{c}
1--- \\
-1-- \\
-\underset{\text { orth }}{-1-1}
\end{array}\right)_{C}=0, \quad\left(\begin{array}{c}
1--- \\
-1-- \\
-\frac{1-}{\text { alias }}
\end{array}\right)_{C}=-6 N \text {, }
\end{aligned}
$$

and

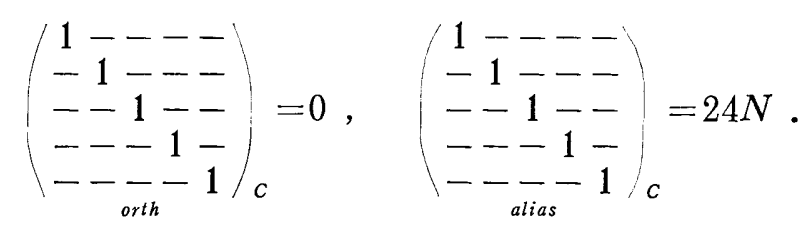

\subsubsection{Ordinary c. g. s. f.'s of O.A.}

In virtue of our O.A. with two levels, any g. s. f.'s containing odd entries can convert the g. s. f.'s which have at most only one odd entry in any column. Furthermore, any c. g. s. f. with only one odd entry in any column can be obtained as the last term of the expansions of the product of the non-zero c. g. s. f.'s which consist of two sorts of c. g. s. f.'s, such that one of these is $\left[1^{s}\right]$ and the other one is the diagonal c. g. s. f.'s such as

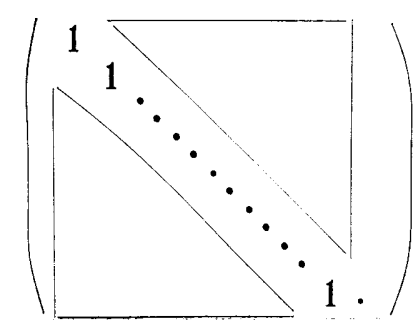




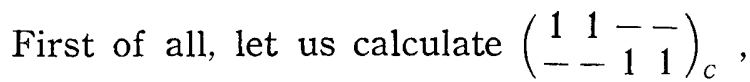

then

$$
\left.\left(\begin{array}{cccc}
1 & 1 & - & - \\
- & - & 1 & 1
\end{array}\right)_{C}=\left(\begin{array}{ll}
1 & 1
\end{array}\right)\left(\begin{array}{ll}
1 & 1
\end{array}\right)-2 \frac{1}{2}\left(\begin{array}{ll}
1 & 1 \\
1 & 1
\end{array}\right)-4 \frac{1}{2}\right)\left(\begin{array}{ccc}
1 & 1 & - \\
- & 1 & 1
\end{array}\right) .
$$

Substituting the results

and

$$
\text { (1) }\left(\begin{array}{ll}
1 & 1 \\
1 & 1
\end{array}\right)=(12)\left(\begin{array}{ll}
1 & 1
\end{array}\right)=-N
$$

$$
\text { (1) }\left(\begin{array}{rrr}
1 & 1 & - \\
- & 1 & 1
\end{array}\right)=\frac{(1)}{(2)}\left(\begin{array}{ll}
1 & - \\
-12 & - \\
- & -1
\end{array}\right)=2 N \text {, }
$$

then we get

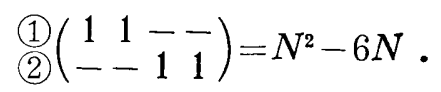

Using these symbolic recurrence procedures, we can calculate the g. s. f.'s in refering to the above c. g. s. f. Tables.

On the other hand, we have a theorem, such that, the order of $N$ of the product, $F(N)$, of non-zero polynomials, $f_{1}(N), \cdots, f_{i}(N)$, is equal to the sum of these orders of the original polynomials. In virtue of the theorem, with respect to the all g. s. m.'s which are derived from the multiplication of several non-zero c. g. s. f.'s, we have

$$
[(s \times h)]^{*}=o\left(N^{s^{*}-h}\right) \text {, }
$$

where $[(s \times h)]^{*}$ is a c. g. s. m. of our O.A. with $s$ rows and $h$ columns and $s^{*}$ stands for the sum of order of $N$ in the original non-zero c. g. $s$. f.'s. Furthermore, we have

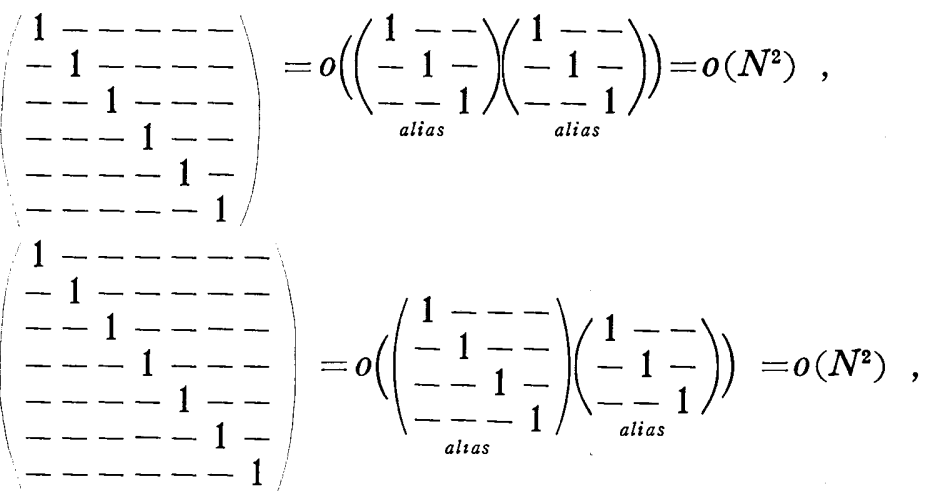

and

$$
\begin{aligned}
& 1---\cdots \\
& -1----- \\
& --1-\ldots-- \\
& ---1--- \\
& ---1--- \\
& ----1- \\
& ------1 \\
& =o\left(\left(\begin{array}{l}
1--- \\
-1-- \\
-\underset{\text { alias }}{-1-1}
\end{array}\right)\left(\begin{array}{c}
1--- \\
-1-- \\
-\underset{\text { alias }}{-}-1-
\end{array}\right)\right)=o\left(N^{2}\right)
\end{aligned}
$$


and, for c. g. s. f. with $h$ columns in diagonal, we have

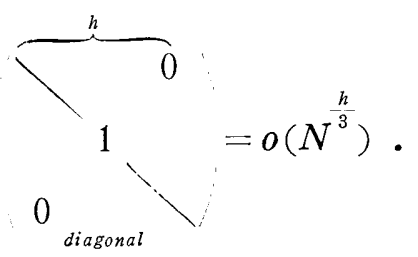

Consequently, refering to the formulas (2.4.9) and (2.4.19), we get

$$
s^{*} \leqq \sum_{i}\left(s_{i 1}^{*}+\frac{s_{i 2}^{*}}{2}\right)
$$

where $s_{i 1}^{*}$ and $s_{i 2}^{*}$ are the sum of even and odd entries of $i$-th original c. g. s. f., for example we have [21111][11] $=N^{(5)} N^{(2)}<21111><11>$, and from this

$$
s^{*} \leqq\left(5-\frac{4}{2}\right)+\left(2-\frac{2}{2}\right)=3+1,
$$

then we get easily

$$
\left[\begin{array}{rrrrr}
2 & 1 & 1 & 1 & 1 \\
- & - & - & 1 & 1
\end{array}\right]=o\left(N^{-1}\right)
$$

and

$$
\left[\begin{array}{cccccc}
2 & 1 & 1 & 1 & 1 & - \\
- & - & - & - & 1 & 1
\end{array}\right]=o\left(N^{-2}\right) .
$$

\section{4 .6 g. s. m.'s of $0 . A$.}

In this section, tables which make possible the calculation of the higher moments of $\lambda_{2}$, will be presented. In virtue of O.A. all g. s. m.'s with $h$ columns can be classified into several g. s. m.'s with $h$ entries which have only one entry in a column as follows.

1 columns:

all entries are even

$$
[(s \times 1)]^{*}=1
$$

2 columns:

all are even

$$
\begin{aligned}
& {[(s \times 2)]^{*}=1} \\
& \text { (1) }\left[\begin{array}{ll}
1 & 1 \\
1 & 1 \\
1 & 1
\end{array}\right)^{*}=1 \\
& \text { (12ias } \\
& \text { (1) }\left[\begin{array}{rr}
1 & 1 \\
\text { (2) } & -
\end{array}\right]^{*}=\frac{-1}{N-1}
\end{aligned}
$$

3 columns: 


$$
\begin{aligned}
& {[(s \times 3)]^{*}=1} \\
& \text { (2) }\left[\begin{array}{ccc}
1 & 1 & - \\
- & - & -
\end{array}\right]^{*}=\frac{-1}{N-1} \\
& \text { (2) }\left[\begin{array}{ccc}
1 & 1 & - \\
- & - & 1
\end{array}\right]^{*}=0 \\
& \text { (1) } 1-\ldots * \\
& \text { (12) }-\frac{1-1}{\text { alias }}=\frac{2}{(N-1)(N-2)} \\
& \text { (1) } 1-\cdots)^{*} \\
& \text { (2) }-1-=0 \\
& \text { (3) }--1
\end{aligned}
$$$$
\text { all are even }
$$

all are even

4 columns:

$$
\begin{aligned}
& {[(s \times 4)]^{*} \quad=1} \\
& \text { (1) }\left[\begin{array}{ccc}
1 & 1 & - \\
- & - & -
\end{array}\right]^{*}=\frac{-1}{N-1} \\
& \text { (1) }\left[\begin{array}{cccc}
1 & 1 & 1 & 1 \\
- & - & - & -
\end{array}\right]^{*}=\frac{3}{(N-1)(N-3)} \\
& \text { (1) }\left[\begin{array}{cccc}
1 & 1 & - & - \\
- & - & 1 & 1
\end{array}\right]^{*}=\frac{N-6}{(N-1)(N-2)(N-3)} \\
& \text { (1) } \left.\frac{1-1-}{-1-1}\right)^{*}=\frac{2}{(N-1)(N-2)} \\
& \text { (1) }\left(\begin{array}{c}
1-1- \\
-\frac{1-1}{\text { (3)th }}
\end{array}\right\rangle^{*}=0 \\
& \text { (1) }(1--\cdots)^{*} \\
& \text { (2) }-1--=\frac{-6}{(N-1)(N-2)} \\
& \text { (3) }-1-1=\overline{(N-1)}(N-2)(N-3) \\
& \text { (4) } \backslash-\overline{\text { alias }}-1 \\
& \text { (1) }(1-\cdots)^{*} \\
& \text { (2) }-1-1-=0 \\
& \text { (4) } \backslash-\frac{-1}{0 . t h}
\end{aligned}
$$

5 and more columns:

For our present purpose with respect to the culculation of the 4 th and less degree moments of $\lambda_{2}$, we have

$$
s^{*} \leqq 4
$$

because $s^{*}$ is the sum of $\left(s_{1}^{*}+s_{2}^{*} / 2\right)$ 's of the original s. f.'s which are constructed by the partitions of the all even partitions of number 8 . In gen- 
eral, we have for $K$-th moment of $\lambda_{2}$

$$
s^{*} \leqq K
$$

because the original s. f.'s are constructed by the partitions of all even partitions of number " $2 K$ ", and $s^{*}$ is equal to the sum of the numbers of even and a half of odd entries in these all s. f.'s.

In virtue of formula (2.4.21) and (2.4.22), we get

$$
[(s \times h)]^{*}=o\left(N^{-(h-k)}\right) \quad \text { for } h>k .
$$

3. Moments of $\lambda_{1 p}$ and $\lambda_{2}$

3.1 Expansions of $\lambda_{1 p}^{S}$ and $\lambda_{2}^{K}$

\subsubsection{Expansions of $\lambda_{1 p}^{S}$}

In virtue of the results in our previous chapter in this paper, we can obtain the $S$-th degree moment (refer to Fisher [5], Robson [7], Behnken [1], and Wishert [11]) of

$$
\begin{aligned}
\dot{V}_{p} & =\frac{1}{N} \sum x_{p n} \dot{v}_{n} \\
& =\frac{1}{N} \sum X_{p n} \\
& =<1_{p}>
\end{aligned}
$$

such that

$$
\operatorname{aver}\left\{\dot{V}_{p}^{s}\right\}=\operatorname{aver}\left\{<1_{p}>^{s}\right\}
$$

refering to the S.F. Table [4].

Henceforth expanding the formula

$$
\lambda_{1 p}^{S}=\left\{\frac{N}{2}\left(\alpha_{p}+\dot{V}_{p}\right)^{2}\right\}^{s} \quad \text { and substituting (3.1.2), we get }
$$

$$
\operatorname{aver}\left\{\lambda_{1 p}\right\}=\left(\begin{array}{c}
N \\
2
\end{array}\right) \sum_{s=0}^{2}\left(\begin{array}{l}
2 \\
s
\end{array}\right) \alpha_{p}^{2-s} \text { aver }\left\{<1_{p}>^{s}\right\}
$$

$$
\operatorname{aver}\left\{\lambda_{1 p}^{2}\right\}=\left(\begin{array}{c}
N \\
2
\end{array}\right)^{2} \sum_{s=0}^{4}\left(\begin{array}{l}
4 \\
s
\end{array}\right) \alpha_{p}^{4-s} \text { aver }\left\{<1_{p}>^{s}\right\}
$$

and 
$(3.1 .3-5)$

$$
\operatorname{aver}\left\{\lambda_{1 p}^{S}\right\}=\left(\begin{array}{c}
N \\
2
\end{array}\right) \sum_{s=0}^{S}\left(\begin{array}{c}
2 S \\
s
\end{array}\right) \alpha_{p}^{2 S-s} \text { aver }<1_{p}>^{s}
$$

Therefore, we have

$(3.1 .4-1)$

$$
\begin{aligned}
\text { aver } \lambda_{1 p} & =\begin{array}{c}
N \\
2
\end{array}\left\{\alpha_{p}^{2}+2 \alpha_{p} \text { aver }\left\langle 1_{p}>+ \text { aver }\left\langle 1_{p}\right\rangle^{2}\right\},\right. \\
& =\frac{N}{2}\left\{\alpha_{p}^{2}+2 \alpha_{p}\left\langle 1_{p}\right\rangle+\frac{1}{N^{\overline{2}}}\left(N\left\langle\left\langle 2_{p}\right\rangle+N(N-1)\left\langle 1_{p} 1_{p}\right\rangle\right)\right\}\right.
\end{aligned}
$$

and

\begin{tabular}{|c|c|c|c|c|}
\hline & $N$ & $N(N-1)$ & $N(N-1)(N-2)$ & $N(N-1)(N-2)(N-3)$ \\
\hline $4 \alpha_{p}^{3} / N$ & $\ll 1_{p} 》$ & & & \\
\hline $6 \alpha_{p^{2}}^{2} / N^{2}$ & $\left\langle 2_{p} 》\right.$ & $\left\langle 1_{p}^{2}\right\rangle$ & & \\
\hline $4 \alpha_{p} / N^{3}$ & $\left\langle 3_{p}\right\rangle$ & $3\left\langle 21_{p} 1_{p}\right\rangle$ & $\left\langle 1_{p}^{3}\right\rangle$ & \\
\hline $1 / N^{4}$ & $\left\langle 4_{p}\right\rangle$ & $4 \ll 3_{p} 1_{p} 》+3\left\langle 2_{p}^{2} 》\right.$ & $6\left\langle 21_{p} 1^{2} 》\right.$ & $\left\langle 1_{p}^{4}\right\rangle$ \\
\hline
\end{tabular}

$$
\begin{aligned}
\operatorname{aver}\left\{\lambda_{1 p}^{2}\right\}= & \left(\frac{N}{2}\right)^{2}\left\{\alpha_{p}^{4}+4 \alpha_{p}^{3} \text { aver }<1_{p}>+6 \alpha_{p}^{2} \text { aver }<1_{p}>^{2}\right. \\
& \left.+4 \alpha_{p} \text { aver }<1_{p}>^{3}+\text { aver }<1_{p}>^{4}\right\} \\
= & \left(\frac{N}{2}\right)^{2} \alpha_{p}^{4}+\left(\frac{N}{2}\right)^{2} \sum_{i=1}^{4} \sum_{j=1}^{4} a_{i} b_{j} c_{i j} .
\end{aligned}
$$

Table 3.1.1-1 Table of the 2nd term of righthand side of the formula (3.1.4-2)

In the above and following Tables, we shall show the double summation $\sum_{i=1}^{I} \sum_{j=1}^{J} a_{i} b_{j} c_{i j}$, as in the Table $3.1 .1-2$.

Table 3.1.1-2 Representation in table of the formula $\sum \sum a_{i} b_{j} c_{i j}$

\begin{tabular}{c|cccccccccc}
\hline & $b_{1}$ & $b_{2}$ & $\cdot$ & $\cdot$ & $\cdot$ & $b_{j}$ & $\cdot$ & $\cdot$ & $\cdot$ & $b_{J}$ \\
\hline$a_{1}$ & $c_{11}$ & $c_{12}$ & $\cdot$ & $\cdot$ & $\cdot$ & $c_{1 j}$ & $\cdot$ & $\cdot$ & $\cdot$ & $c_{1 J}$ \\
$a_{2}$ & $c_{21}$ & $c_{22}$ & $\cdot$ & $\cdot$ & $\cdot$ & $c_{2 j}$ & $\cdot$ & $\cdot$ & $\cdot$ & $c_{2 J}$ \\
$\cdot$ & $\cdot$ & $\cdot$ & $\cdot$ & $\cdot$ & $\cdot$ & $\cdot$ & $\cdot$ & $\cdot$ & $\cdot$ & $\cdot$ \\
$\cdot$ & $\cdot$ & $\cdot$ & $\cdot$ & $\cdot$ & $\cdot$ & $\cdot$ & $\cdot$ & $\cdot$ & $\cdot$ & $\cdot$ \\
$\cdot$ & $\cdot$ & $\cdot$ & $\cdot$ & $\cdot$ & $\cdot$ & $\cdot$ & $\cdot$ & $\cdot$ & $\cdot$ & $\cdot$ \\
$a_{i}$ & $c_{i 1}$ & $c_{i 2}$ & $\cdot$ & $\cdot$ & $\cdot$ & $c_{i j}$ & $\cdot$ & $\cdot$ & $\cdot$ & $c_{i J}$ \\
$\cdot$ & $\cdot$ & $\cdot$ & $\cdot$ & $\cdot$ & $\cdot$ & $\cdot$ & $\cdot$ & $\cdot$ & $\cdot$ & $\cdot$ \\
$\cdot$ & $\cdot$ & $\cdot$ & $\cdot$ & $\cdot$ & $\cdot$ & $\cdot$ & $\cdot$ & $\cdot$ & $\cdot$ & $\cdot$ \\
$\cdot$ & $\cdot$ & $\cdot$ & $\cdot$ & $\cdot$ & $\cdot$ & $\cdot$ & $\cdot$ & $\cdot$ & $\cdot$ & $\cdot$ \\
$a_{I}$ & $c_{I 1}$ & $c_{I 2}$ & $\cdot$ & $\cdot$ & $\cdot$ & $c_{I j}$ & $\cdot$ & $\cdot$ & $\cdot$ & $c_{I J}$ \\
\hline
\end{tabular}


$(3.1 .4-3)$

$$
\begin{aligned}
& \text { aver }\left\{\lambda_{1_{p}^{3}}^{3}\right\}=\left(\begin{array}{c}
N \\
2
\end{array}\right)^{3}\left\{\alpha_{p}^{6}+6 \alpha_{p}^{5} \text { aver }<1_{p}>+\left(\begin{array}{l}
6 \\
2
\end{array}\right) \alpha_{p}^{4} \text { aver }<1_{p}>^{2}\right. \\
& +\left(\begin{array}{c}
6 \\
3
\end{array}\right) \alpha_{p}^{3} \text { aver }<1_{p}>^{3}+\left(\begin{array}{l}
6 \\
4
\end{array}\right) \alpha_{p}^{2} \text { aver }<1_{p}>^{4}+6 \alpha_{p} \text { aver }<1_{p}>^{5} \\
& \left.+ \text { aver }<1_{p}>^{6}\right\} \\
& \quad=\left(\frac{N}{2}\right)^{3} \alpha_{p}^{6}+\left(\frac{N}{2}\right)^{3} \sum_{j=1}^{6} \sum_{i=1}^{6} a_{i} b_{j} c_{i j},
\end{aligned}
$$

\begin{tabular}{|c|c|c|c|c|c|c|}
\hline & $N^{(1)}$ & $N^{(2)}$ & $N^{(3)}$ & $N^{(4)}$ & $N^{(5)}$ & $N^{(6)}$ \\
\hline$\frac{6}{N} \alpha_{p}{ }^{5}$ & $\left\langle 1_{p}\right\rangle$ & & & & & \\
\hline$\frac{15}{N^{2}} \alpha_{p}^{4}$ & $\langle 2 p 》$ & $\left\langle 1 p^{2} 》\right.$ & & & & \\
\hline$\frac{20}{N^{3}} \alpha_{p^{3}}$ & $\left\langle 3_{p} 》\right.$ & $3 \ll 2_{p} 1_{p} 》$ & $\left\langle 1_{p^{3}}\right\rangle$ & & & \\
\hline$\frac{15}{N^{4}} \alpha_{p}^{2}$ & $\ll 4_{p} 》$ & $\begin{array}{l}4 \ll 3_{p} 1_{p} 》 \\
\left.3 \ll 2_{p}^{2}\right\rangle\end{array}$ & $6\left\langle 2_{p} 1_{p}^{2} 》\right.$ & $\left\langle 1_{p}^{4}\right\rangle$ & & \\
\hline$\frac{6}{N^{5}} \alpha_{p}$ & $\left\langle 5_{p} 》\right.$ & $\begin{array}{c}5 \ll 4_{p} 1_{p} 》 \\
10 \ll 3_{c} 2_{p} 》\end{array}$ & $\begin{array}{l}10 \ll 3_{p} 1_{p}^{2} 》 \\
15 \ll 2_{p}^{2} 1_{p} 》\end{array}$ & $10 \ll 2_{p} 1_{p}^{3} 》$ & $\ll 1_{p}^{5} 》$ & \\
\hline$\frac{1}{N^{6}}$ & $\ll 6_{p} 》$ & $\begin{array}{c}6 \ll 5_{p} 1_{p} 》 \\
\left.15 \ll 4_{p} 2_{p}\right\rangle \\
\left.10 \ll 3_{p}{ }^{2}\right\rangle\end{array}$ & $\begin{array}{l}15 \ll 4_{p} 1_{p}{ }^{2} 》 \\
\left.66 \ll 3_{p} 2_{p} 1_{p}\right\rangle \\
15\left\langle 2_{p}{ }^{3}\right\rangle\end{array}$ & $\begin{array}{l}\left.20 \ll 3 p 1_{p}^{3}\right\rangle \\
\left.45 \ll 2 p_{p}^{2} 1_{p}^{2}\right\rangle\end{array}$ & $15 \ll 2_{p} 1_{p}^{4} 》$ & $\left\langle 1 p^{6}\right\rangle$ \\
\hline
\end{tabular}

Table 3.1.1-3 Representation of the $\sum \sum a_{i} b_{j} c_{i j}$ term in the formula (3.1.4-3)

(3.1.4-4) $\quad$ aver $\left\{\lambda_{1 p}^{4}\right\}=\left(\frac{N}{2}\right)^{4}\left\{\alpha_{p}^{8}+8 \alpha_{p}^{7}\right.$ aver $<1_{p}>+\left(\begin{array}{l}8 \\ 2\end{array}\right) \alpha_{p}^{6}$ aver $<1_{p}>^{2}$

$$
\begin{aligned}
& +\left(\begin{array}{l}
8 \\
3
\end{array}\right) \alpha_{p}^{5} \text { aver }<1_{p}>^{3}+\left(\begin{array}{l}
8 \\
4
\end{array}\right) \alpha_{p}^{4} \text { aver }<1_{p}>^{4}+\left(\begin{array}{l}
8 \\
5
\end{array}\right) \alpha_{p}^{3} \text { aver }<1_{p}>^{5} \\
& \left.+\left(\begin{array}{l}
8 \\
6
\end{array}\right) \alpha_{p}^{2} \text { aver }<1_{p}>^{6}+8 \alpha_{p} \text { aver }<1_{p}>^{7}+\text { aver }<1_{p}>^{8}\right\} \\
& =\left(\frac{N}{2}\right)^{4} \alpha_{p}^{8}+\left(\frac{N}{2}\right)^{4} \sum_{i=1}^{8} \sum_{j=1}^{8} a_{i} b_{j} c_{i j}
\end{aligned}
$$

and

$(3.1 .4-5)$

$$
\begin{aligned}
\operatorname{aver}\left\{\lambda_{1 p}^{S}\right\}= & \left(\frac{N}{2}\right)^{S}\left\{\alpha_{p}^{2 S}+\left(\begin{array}{c}
2 S \\
1
\end{array}\right) \alpha_{p}^{2 S-1} \text { aver }<1_{p}>+\right. \\
& \left.\cdots+\text { aver }<1_{p}>^{2 S}\right\} \\
= & \left(\frac{N}{2}\right)^{S} \alpha_{p}^{2 S}+\left(\frac{N}{2}\right)^{S} \sum_{i=1}^{2 S} \sum_{j=1}^{2 S} a_{i} b_{j} c_{i j} .
\end{aligned}
$$


Table 3.1.1-4 Representation of the $\left(\sum \Sigma a_{i} b_{j} c_{i j}\right)$ term of the formula (3.1.4-4)

\begin{tabular}{|c|c|c|c|c|c|c|c|c|}
\hline & $N$ & $N^{(2)}$ & $N^{(3)}$ & $N^{(4)}$ & $N^{(5)}$ & $N^{(6)}$ & $N^{(7)}$ & $N^{(8)}$ \\
\hline$\frac{8}{N} \alpha_{p}^{7}$ & $\left\langle 1_{p}\right\rangle$ & & & & & & & \\
\hline$\frac{28}{N^{1}} \alpha_{p}^{6}$ & $\left\langle 2_{p}\right\rangle$ & $\left\langle 1 p^{2} 》\right.$ & & & & & & \\
\hline$\frac{56}{N^{3}} \alpha_{p}^{5}$ & $\left\langle 3_{p}\right\rangle$ & $3 \ll 2_{p} 1_{p} 》$ & $\left\langle 1 p^{3} \rrbracket\right.$ & & & & & \\
\hline$\frac{70}{N^{4}} \alpha_{p^{4}}$ & $《 4_{p} 》$ & $\begin{array}{l}\left.4 \ll 3_{p} 1_{p}\right\rangle \\
\left.3 \ll 2_{p}^{2}\right\rangle\end{array}$ & $6\left\langle 22_{p} 1_{p}^{2} 》\right.$ & $\left\langle 1_{p}^{4}\right\rangle$ & & & & \\
\hline$\frac{56}{N^{5}} \alpha_{p^{3}}$ & $\left\langle 5_{p}\right\rangle$ & $\begin{array}{l}\left.5 \ll 4_{p} 1_{p}\right\rangle \\
10\left\langle 3_{p} 2_{p}\right\rangle\end{array}$ & $\begin{array}{l}10 \ll 3{ }_{p} 1_{p}^{2} 》 \\
\left.15 \ll 2 p_{p}^{2} 1_{p}\right\rangle\end{array}$ & $\left.10 \ll 2_{p} 1_{p}^{3}\right\rangle$ & $\left\langle 11_{p}^{5} 》\right.$ & & & \\
\hline$\frac{28}{N^{6}} \alpha_{p}^{2}$ & $\left\langle\overline{\left.\sigma_{p}\right\rangle}\right\rangle$ & $\begin{array}{l}6\left\langle 5_{p} 1_{p}\right\rangle \\
15\left\langle 4_{p} 2_{p}\right\rangle \\
10\left\langle 3_{p}^{2}\right\rangle\end{array}$ & $\mid \begin{array}{l}15 \ll 4_{p} 1_{p}^{2} 》 \\
\left.60 \ll 3_{p} 2_{p} 1_{p}\right\rangle \\
\left.15 \ll 2_{p}^{3}\right\rangle \\
\end{array}$ & $\begin{array}{l}\left.20 \ll 3_{p} 1_{p}^{3}\right\rangle \\
45\left\langle 2_{p}{ }^{2} 1_{p}^{2}\right\rangle\end{array}$ & $15\left\langle 22_{p} 1_{p} 4\right\rangle$ & $\left\langle 1_{p}^{6}\right\rangle$ & & \\
\hline$\frac{8}{N^{7}} \alpha_{p^{1}}$ & $\left\langle 7_{p}\right\rangle$ & $\begin{array}{l}\left.7 \ll 6_{p} 1_{p}\right\rangle \\
21\left\langle 5_{p} 2_{p}\right\rangle \\
\left.35 \ll 4_{p} 3_{p}\right\rangle\end{array}$ & $\left\{\begin{array}{c}21 \ll 5_{p} 1_{p}^{2} 》 \\
\left.105 \ll 4_{p} 2_{p} 1_{p}\right\rangle \\
70 \ll 3_{p} 1_{p}^{2} 》 \\
105 \ll 3_{p} 2_{p}^{2} 》\end{array}\right.$ & $\mid \begin{array}{l}\left.35 \ll 4_{p} 1_{p}{ }^{3}\right\rangle \\
\left.210 \ll 3_{p} 2_{p} 1_{p}^{2}\right\rangle \\
\left.105 \ll 2_{p}^{3} 1_{p}\right\rangle\end{array}$ & $\begin{array}{c}\left.35 \ll 3 p^{1} p^{4}\right\rangle \\
105\left\langle 2_{p}^{2} 1_{p^{3}}\right\rangle\end{array}$ & $21\left\langle 2{ }_{p} 1_{p}{ }^{5}\right\rangle$ & $《 1_{p}{ }^{7} 》$ & \\
\hline$\frac{1}{N^{8}}$ & $\left\langle 8_{p}\right\rangle$ & $\begin{array}{l}8\left\langle 7_{p} 1_{p}\right\rangle \\
28\left\langle 6_{p} 2_{p}\right\rangle \\
56\left\langle 5_{p} 3_{p}\right\rangle \\
35\left\langle 4_{p}^{2}\right\rangle\end{array}$ & $\left\{\begin{array}{c}28 \ll 6_{p} 1_{p}{ }^{2} 》 \\
168 \ll 5_{p} 2_{p} 1_{p} 》 \\
\left.280 \ll 4_{p} 3_{p} 1_{p}\right\rangle \\
210 \ll 4_{p} 2_{p}^{2} 》 \\
280 \ll 3_{p}^{2} 2_{p} 》\end{array}\right.$ & $\begin{array}{l}\left.56 \ll 5_{p} 1_{p}^{3}\right\rangle \\
\left.420 \ll 4_{p} 2_{p} 1_{p}^{2}\right\rangle \\
\left.280 \ll 3_{p}^{2} 1_{p}^{2}\right\rangle \\
840\left\langle 3_{p} 2_{p}^{2} 1_{p}\right\rangle \\
\left.105 \ll 2_{p}^{4}\right\rangle\end{array}$ & $\left\{\begin{array}{c}70 \ll 4_{p} 1_{p}{ }^{4} 》 \\
560 \ll 3_{p} 2_{p} 1_{p}^{3} 》 \\
420\left\langle 2_{p}{ }^{3} 1_{p}^{2} 》\right.\end{array}\right.$ & $\begin{array}{c}56 \ll 3_{p} 1_{p} 5 》 \\
\left.210 \ll 2_{p}^{2} 1_{p}^{4}\right\rangle\end{array}$ & $\left.28 \ll 2_{p} 1_{p}{ }^{6}\right\rangle$ & $\left\langle 1_{p}^{8}\right\rangle$ \\
\hline
\end{tabular}

Table 3.1.1-5 Representation of the $\left(\sum \Sigma a_{i} b_{j} c_{i j}\right)$ term of the formula $(3.1 .4-5)$

\begin{tabular}{|c|c|c|c|c|c|c|}
\hline & $N^{(1)}$ & - & - & - & - & $N^{(2 S)}$ \\
\hline$\frac{1}{N}\left(\begin{array}{c}2 S \\
1\end{array}\right) \alpha_{p}{ }^{2 S-1}$ & $\left\langle 1_{p}\right\rangle$ & & & & & \\
\hline$\frac{1}{N^{2}}\left(\begin{array}{c}2 S \\
2\end{array}\right) \alpha_{p}{ }^{2 S-2}$ & $\left\langle 2_{p} 》\right.$ & • & - & - & • & \\
\hline$\frac{1}{N^{3}}\left(\begin{array}{c}2 S \\
3\end{array}\right) \alpha_{p}{ }^{2 S-3}$ & $\left\langle 3_{p}\right\rangle$ & - & - & - & • & \\
\hline$\dot{.}$ & $\dot{.}$ & • & • & • & • & \\
\hline$\frac{1}{\bar{N}^{2}}\left(\begin{array}{l}2 S \\
2 S\end{array}\right)$ & $\left\langle 2 S_{p} 》\right.$ & • & - & $\theta^{\circ}$ & - & $\ll 1_{p}^{2 S} 》$ \\
\hline
\end{tabular}

\subsubsection{Expansions of $\lambda_{2}^{K}$}

As we have seen in Lemma 2 of the previous paper [12], we have another statistic

$$
\lambda_{2}=\frac{N}{2} \sum_{\phi=1}^{\oplus} \dot{V}_{\phi}^{2}
$$


where

$$
\Phi=N-P \text {. }
$$

In virtue of the S.F. Table [4], we can expand the higher powers of $\lambda_{2}$, as follows,

$$
\begin{aligned}
& \lambda_{2}=\frac{N}{2}[2] \\
& \lambda_{2}^{2}=\left(\begin{array}{c}
N \\
2
\end{array}\right)^{2}\{[4]+[22]\} \\
& \lambda_{2}^{3}=\left(\begin{array}{c}
N \\
2
\end{array}\right)^{3}\{[6]+3[42]+[222]\} \\
& \lambda_{2}^{4}=\left(\begin{array}{c}
N \\
2
\end{array}\right)^{4}\left\{[8]+4[62]+3\left[4^{2}\right]+6\left[42^{2}\right]+\left[2^{4}\right]\right\},
\end{aligned}
$$

where square brackets [ ]'s are symmetric functions of $\dot{V}_{1}^{2}, \dot{V}_{2}^{2}, \ldots, \dot{V}_{o}^{2}$, such that

$$
[a b \cdots e]=\sum_{f}^{+} \dot{V}_{f}^{a} \dot{V}_{g}^{b} \cdots \dot{V}_{k}^{e} .
$$

In general, we have

$$
\lambda_{2}^{K}=\left(\frac{N}{2}\right)^{K} \sum_{t}\left(\frac{K !}{a ! \cdots e !}\right)\left(\frac{1}{\alpha ! \cdots \varepsilon !}\right)\left[(2 a)^{\alpha} \cdots(2 e)^{\varepsilon}\right],
$$

where $\sum_{i}$ stands a summation over all even possible partitions of the number $2 K,\left[(2 a)^{\alpha} \cdots(2 e)^{\varepsilon}\right]$. Substituting the relation $\dot{V}_{f}^{2}=\left\langle 1_{f}\right\rangle^{2}$ to the above square brackets, we get
$(3.1 .9-1)$
$\left.[2]=\sum_{f}<1_{f}\right\rangle^{2}$
$(3.1 .9-2)$
$[4]=\sum_{f}<1_{f}>^{4}$
$(3.1 .9-3)$
$[22]=\sum_{f}^{ \pm}\left\langle 1_{f}\right\rangle^{2}\left\langle 1_{g}\right\rangle^{2}$
$(3.1 .9-4)$
$[6]=\sum_{f}<1_{f}>^{6}$
$(3.1 .9-5)$
$[42]=\sum_{f}^{+}\left\langle 1_{f}\right\rangle^{4}\left\langle 1_{g}\right\rangle^{2}$
$(3.1 .9-6)$
$[222]=\sum_{f}^{ \pm}\left\langle 1_{f}\right\rangle^{2}\left\langle 1_{g}\right\rangle^{2}\left\langle 1_{h}\right\rangle^{2}$
$(3.1 .9-7)$
$[8]=\Sigma_{f}<1_{f}>^{8}$
$(3.1 .9-8)$
$\left.[62]=\sum_{f}^{+}<1_{f}\right\rangle^{6}<1_{g}>^{2}$
$[44]=\sum_{f}^{*}\left\langle 1_{f}\right\rangle^{4}\left\langle 1_{g}\right\rangle^{4}$
$(3.1 .9-10)$
$[422]=\sum_{f}^{*}\left\langle 1_{f}>^{4}<1_{g}>^{2}<1_{h}>^{2}\right.$

and

$$
[2222]=\sum_{f}^{+}\left\langle 1_{f}>^{2}\left\langle 1_{g}\right\rangle^{2}\left\langle 1_{h}\right\rangle^{2}<1_{i}>^{2} .\right.
$$

These multiplication $(3.1 .9-3,5,6,8,9$, and 10$)$ of powers of angle brackets such as $<>$ 's are tabulated in Table 3.1.2. In this table, we shall mean that 
Table 3.1.2 Multiplications of the angle brackets, $<>$ 's

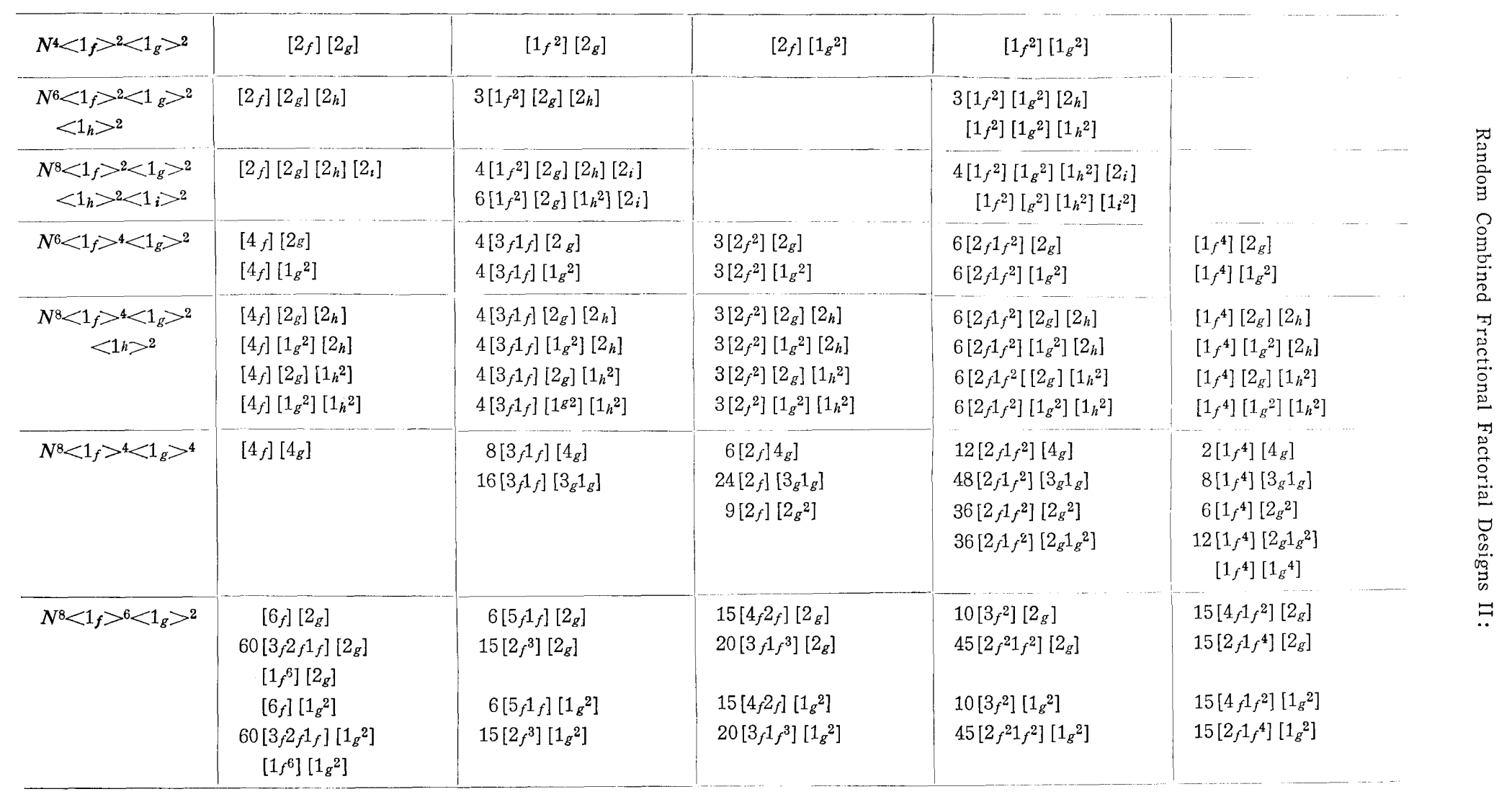


(3.1.10)

1 st column $=$ sum of terms of $2 \mathrm{nd}, 3 \mathrm{rd}, \cdots$, and last columns for example

$$
\left.N^{2}<1_{f}\right\rangle^{2}<1_{g}>^{2}=\left[2_{f}\right]\left[2_{g}\right]+\left[1_{f}{ }^{2}\right]\left[2_{g}\right]+\left[2_{f}\right]\left[1_{g}{ }^{2}\right] \div\left[1_{f}{ }^{2}\right]\left[1_{g}{ }^{2}\right] .
$$

Furthermore the multiplications of brackets are given by the method such that

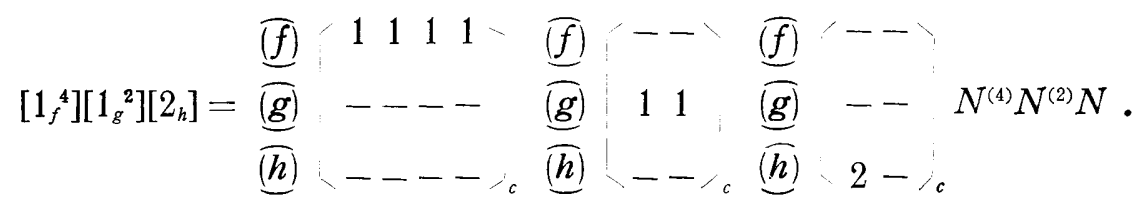

3.1.3 1st, 2nd, 3rd and 4th moments of $\lambda_{2}$

In virtue of these results, we can write down the moments of $\lambda_{2}$, as follows. We shall present the moments of $\lambda_{2}$ in the style of tables, as we have seen in the moments of $\lambda_{1 p}$. In these tables, we shall mean $\sum_{i=1}^{I} \sum_{j=1}^{J} a_{i} b_{j} c_{i j}$, where $a_{i}$ is $i$-th row entry in the 1 st column $b_{j}$ is $j$-th column entry in the 1 st row and $c_{i j}$ is the sum of $i$-th row and $j$-th column entry. In these expansions we shall use the relation

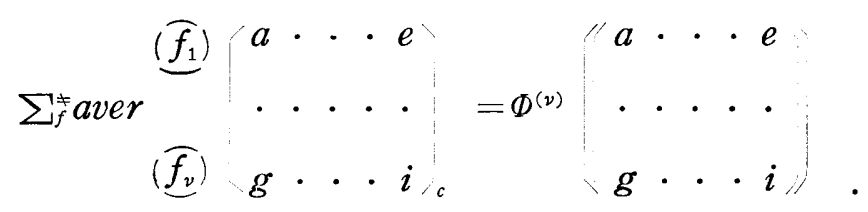

Furthermore, the coefficients of these g. s. m.'s are obtained by the combinatorial rules which can be seen in the section 2.1.2.

Then we get the higher moments of $\lambda_{2}$, as follows,

$$
\begin{aligned}
& \operatorname{aver}\left\{\lambda_{2}\right\}=\frac{N}{2} \sum_{f} \text { aver }\left\{\left\langle 1_{f}\right\rangle^{2}\right\} \\
& =\frac{1}{2} \sum_{j=1}^{2} a_{1} b_{j} c_{1 j},
\end{aligned}
$$

Table 3.1.3-1 Representation of the formula (3.1.12-1)

\begin{tabular}{c|c|c} 
& $N^{(1)-1}$ & $N^{(2)-1}$ \\
\hline 0 & {$\left[\left[\begin{array}{c|c}2--] \\
-\cdots\end{array}\right]\right.$} & {$\left[\left[\begin{array}{ll}1 & 1\end{array}\right]\right]$} \\
\hline
\end{tabular}

$$
\begin{aligned}
\operatorname{aver}\left\{\lambda_{2}^{2}\right\} & \left.=\left(\frac{N}{2}\right)^{2}\left\{\text { aver } \sum_{f}<1_{f}\right\rangle^{4}+\operatorname{aver} \Sigma^{\ddagger}\left\langle 1_{f}>^{2}<1_{g}\right\rangle^{2}\right\} \\
& =\left(\frac{1}{2}\right)^{2} \sum_{j=1}^{4} \sum_{i=1}^{2} a_{i} b_{j} c_{i j}
\end{aligned}
$$


Random Combined Fractional Factorial Designs II:

Table 3.1.3-2 Representation of the formula (3.1.12-2)

\begin{tabular}{|c|c|c|c|c|}
\hline & $N N^{-2}$ & $\frac{N(N-1)}{N^{2}}$ & $\frac{N(N-1)(N-2)}{N^{2}}$ & $\frac{N(N-1)(N-2)(N-3)}{N^{2}}$ \\
\hline$\Phi$ & {$[[4-]]$} & $\begin{array}{l}4\left[\left[\begin{array}{ll}3 & 1 \\
-\end{array}\right]\right] \\
3\left[\left[\begin{array}{ll}2 & 2 \\
- & -\end{array}\right]\right]\end{array}$ & $6\left[\left[\begin{array}{lll}2 & 1 & 1 \\
- & -\end{array}\right]\right]$ & {$\left[\left[\begin{array}{llll}1 & 1 & 1 & 1 \\
\hdashline\end{array}\right]\right]$} \\
\hline$\Phi^{(2)}$ & {$\left[\left[\begin{array}{ll}2- \\
2-\end{array}\right]\right]$} & $\begin{array}{r}{\left[\left[\begin{array}{ll}2 & - \\
-2\end{array}\right]\right]} \\
4\left[\left[\begin{array}{ll}1 & 1 \\
2 & -\end{array}\right]\right] \\
2\left[\left[\begin{array}{ll}1 & 1 \\
1 & 1\end{array}\right]\right]\end{array}$ & $\begin{array}{l}2\left[\left[\begin{array}{lll}1 & 1 & - \\
- & -2\end{array}\right]\right] \\
4\left[\left[\begin{array}{lll}1 & 1 & - \\
- & 1 & 1\end{array}\right]\right]\end{array}$ & {$\left[\left[\begin{array}{llll}1 & 1 & - & -\end{array}\right]\right]$} \\
\hline
\end{tabular}

$(3.1 .12-3) \quad$ aver $\left\{\lambda_{2}^{3}\right\}=\left(\frac{1}{2}\right)^{3} \sum_{j=1}^{6} \sum_{i=1}^{3} a_{i} b_{j} c_{i j}$,

and

(3.1.12-4) $\quad$ aver $\left\{\lambda_{2}^{4}\right\}=\left(\frac{1}{2}\right)^{4} \sum_{j=1}^{8} \sum_{i=1}^{4} a_{i} b_{j} c_{i j}$ 
Table 3.1.3-3 Representation of the expansion of formula 3.1.12-3, aver $\left\{\lambda_{2}{ }^{3}\right\}$

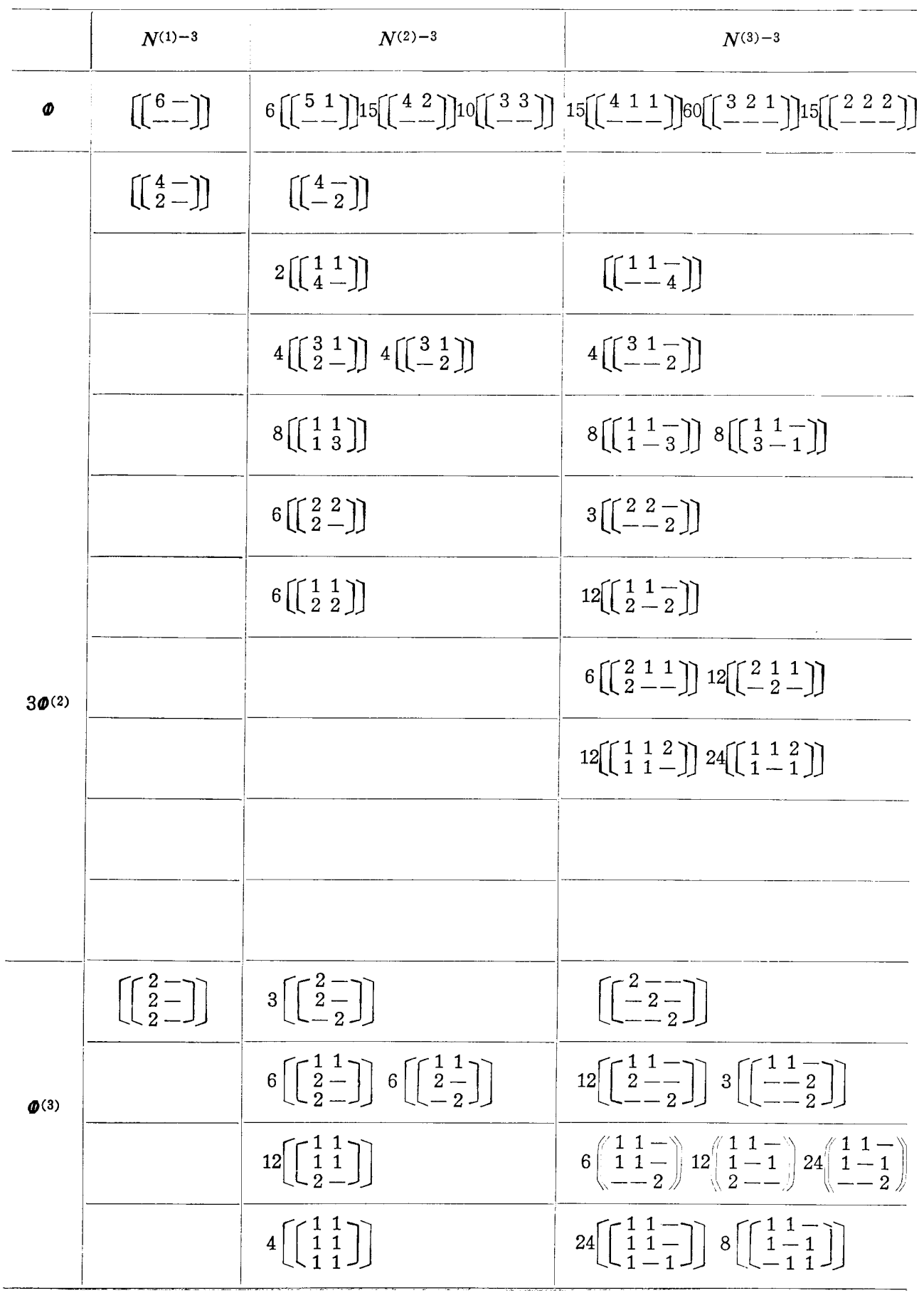




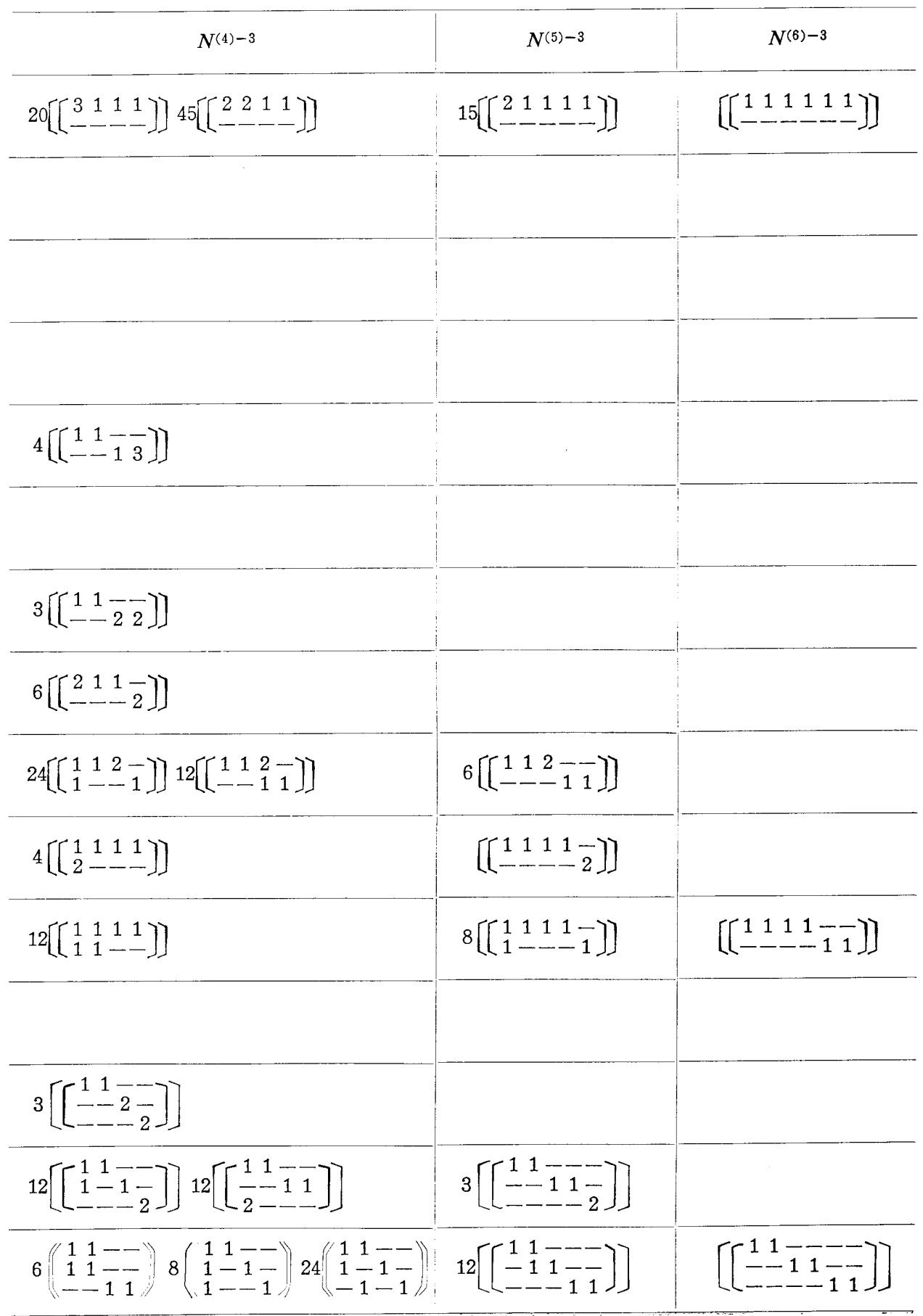


Table 3.1.3-4 Expansion of the formula 3.1.12-4, aver $\left\{\lambda_{2} 4\right\}$

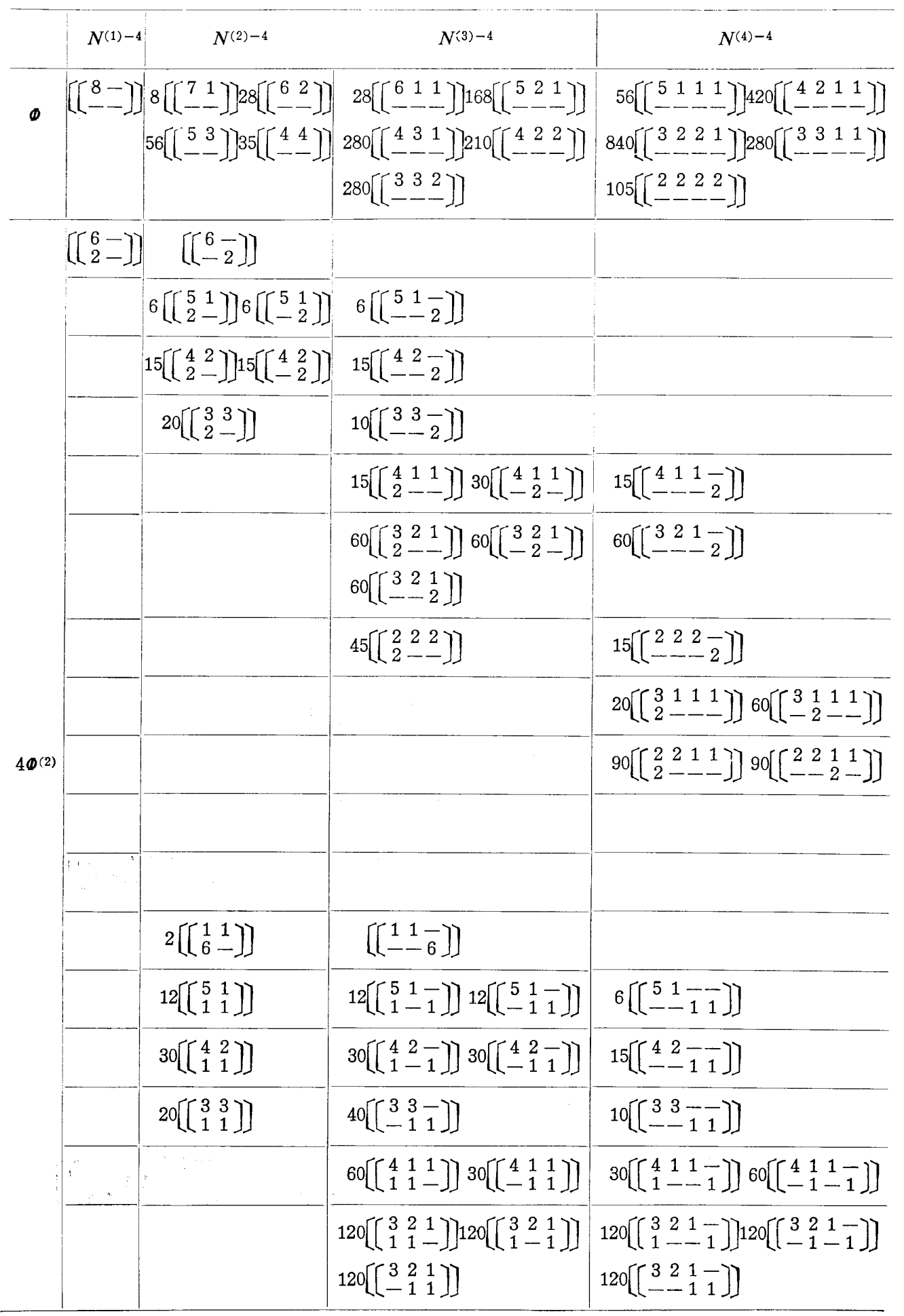




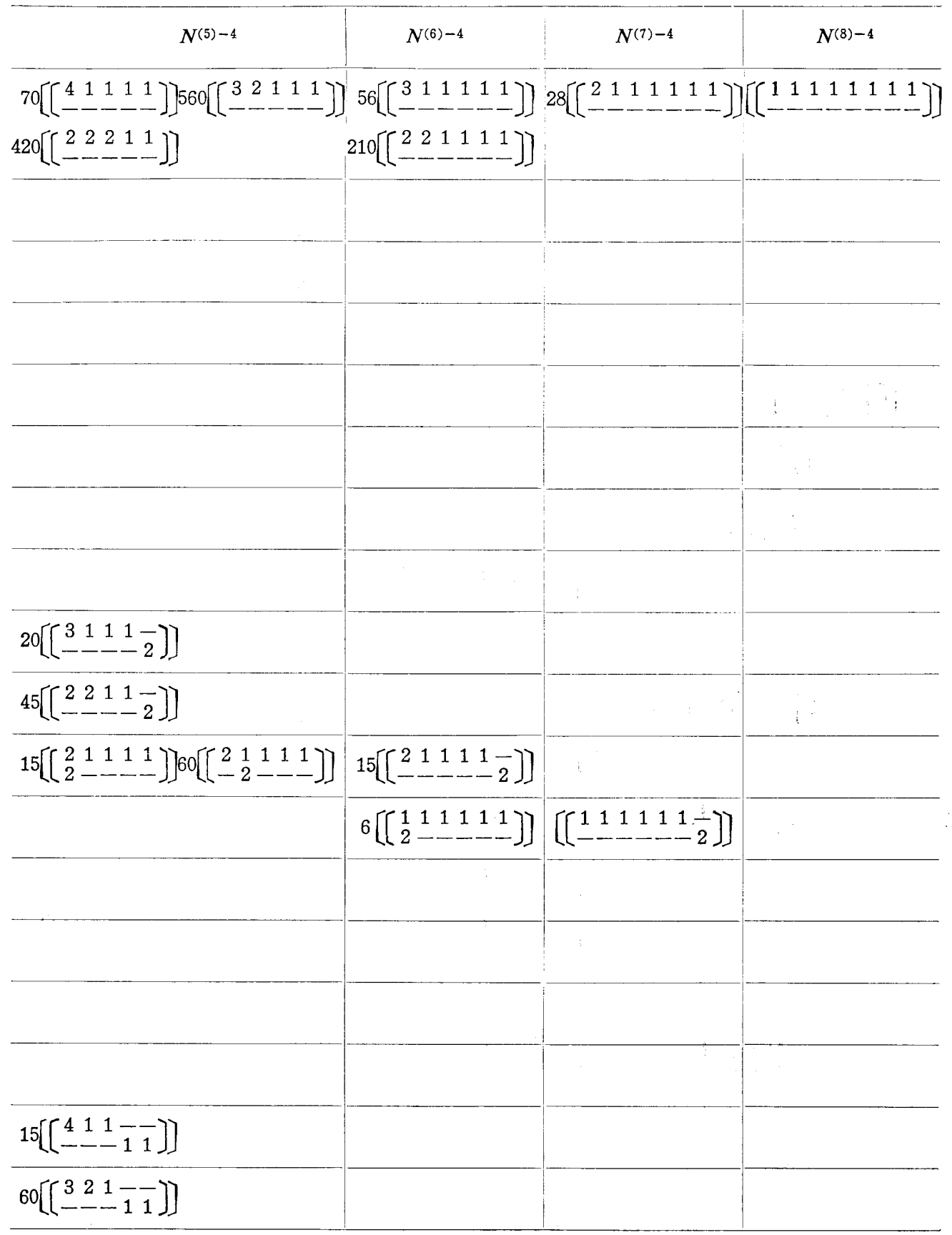


Table 3.1.3-5 Continue

\begin{tabular}{|c|c|c|c|c|}
\hline & $N^{(1)-4}$ & $N^{(2)-4}$ & $N^{(3)-4}$ & $N^{(4)-4}$ \\
\hline & & & $90\left[\left[\begin{array}{lll}2 & 2 & 2 \\
1 & 1 & -\end{array}\right]\right]$ & $90\left[\left[\begin{array}{llll}2 & 2 & 2 & - \\
1 & - & - & -1\end{array}\right]\right.$ \\
\hline & & & & $120\left[\left[\begin{array}{llll}3 & 1 & 1 & 1 \\
1 & 1 & - & -\end{array}\right]\right] 120\left[\left[\left[\begin{array}{cccc}3 & 1 & 1 & 1 \\
-1 & 1 & 1 & -\end{array}\right]\right]\right.$ \\
\hline & & & & $\begin{array}{l}90\left[\left[\begin{array}{llll}2 & 2 & 1 & 1 \\
1 & 1 & -1\end{array}\right]\right] 90\left[\left[\begin{array}{llll}2 & 2 & 1 & 1 \\
- & -1 & 1\end{array}\right]\right] \\
360\left[\left[\begin{array}{llll}2 & 2 & 1 & 1 \\
- & 1 & 1 & -\end{array}\right]\right]\end{array}$ \\
\hline \multirow{13}{*}{$3 \Phi^{(2)}$} & {$\left[\begin{array}{l}4- \\
4-\end{array}\right]$} & {$\left[\left[\frac{4-\overline{4}}{-}\right]\right]$} & & \\
\hline & & $8\left[\left[\begin{array}{ll}3 & 1 \\
4 & -\end{array}\right]\right] 8\left[\left[\begin{array}{ll}3 & 1 \\
-4\end{array}\right]\right]$ & 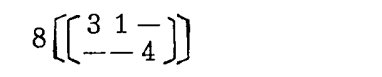 & \\
\hline & & $12\left[\left[\begin{array}{ll}2 & 2 \\
4 & -\end{array}\right]\right]$ & 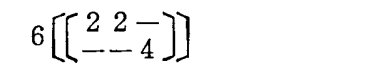 & \\
\hline & & & $24\left[\left[\begin{array}{lll}1 & 1 & 2 \\
4 & - & -\end{array}\right]\right] 12\left[\left[\begin{array}{lll}1 & 1 & 2 \\
- & -4\end{array}\right]\right]$ & $12\left[\left[\begin{array}{lll}1 & 1 & 2 \\
- & - & -4\end{array}\right]\right.$ \\
\hline & & & & $8\left[\left[\begin{array}{llll}1 & 1 & 1 & 1 \\
4 & - & & -\end{array}\right]\right]$ \\
\hline & & $16\left[\left[\begin{array}{ll}3 & 1 \\
3 & 1\end{array}\right]\right] 16\left[\left[\begin{array}{ll}3 & 1 \\
1 & 3\end{array}\right]\right]$ & $\begin{array}{l}16\left[\left[\begin{array}{lll}3 & 1 & - \\
- & 3 & 1\end{array}\right]\right] 16\left[\left[\left[\begin{array}{lll}3 & 1 & - \\
- & 1 & 3\end{array}\right]\right]\right. \\
16\left[\left[\begin{array}{lll}3 & 1 & - \\
3 & -1\end{array}\right]\right] 16\left[\left[\begin{array}{lll}3 & 1-\overline{1} \\
1 & - & -3\end{array}\right]\right]\end{array}$ & $16\left[\left[\begin{array}{lll}3 & 1-- & -\end{array}\right]\right]$ \\
\hline & & $48\left[\left[\begin{array}{ll}2 & 2 \\
3 & 1\end{array}\right]\right]$ & 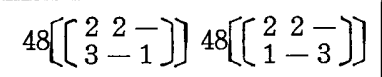 & $24\left[\left[\begin{array}{llll}2 & 2 & - & - \\
- & -3 & 1\end{array}\right]\right]$ \\
\hline & & & $\begin{array}{l}96\left[\left[\begin{array}{lll}2 & 1 & 1 \\
3 & 1 & -\end{array}\right]\right] 96\left[\left[\begin{array}{lll}2 & 1 & 1 \\
-3 & 1\end{array}\right]\right] \\
96\left[\left[\begin{array}{lll}2 & 1 & 1 \\
1 & 3 & -\end{array}\right]\right]\end{array}$ & $\begin{array}{l}48\left[\left[\begin{array}{llll}2 & 1 & 1 & - \\
3 & - & -1\end{array}\right]\right] 96\left[\left[\begin{array}{llll}2 & 1 & 1 & - \\
- & 3 & -1\end{array}\right]\right] \\
48\left[\left[\begin{array}{llll}2 & 1 & 1 & - \\
1 & - & -3\end{array}\right]\right] 96\left[\left[\begin{array}{cccc}2 & 1 & 1 & - \\
- & 1 & -3\end{array}\right]\right]\end{array}$ \\
\hline & & & & $96\left[\left[\begin{array}{llll}1 & 1 & 1 & 1 \\
3 & 1 & - & -\end{array}\right]\right]$ \\
\hline & & $18\left[\left[\begin{array}{ll}2 & 2 \\
2 & 2\end{array}\right]\right]$ & $36\left[\left[\begin{array}{ll}2 & 2 \\
2 & -2\end{array}\right]\right]$ & 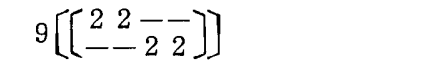 \\
\hline & & & $\left.144\left[\left[\begin{array}{lll}2 & 1 & 1 \\
2 & 2 & -\end{array}\right]\right] 72\left[\left[\begin{array}{lll}2 & 1 & 1 \\
- & 2 & 2\end{array}\right]\right]\right]$ & $72\left[\left[\begin{array}{llll}2 & 1 & 1 & - \\
2 & - & -2\end{array}\right]\right] 144\left[\left[\begin{array}{llll}2 & 1 & 1 & - \\
- & -2 & - & -2\end{array}\right]\right]$ \\
\hline & & & & $72\left[\left[\begin{array}{llll}1 & 1 & 1 & 1 \\
2 & 2 & - & -\end{array}\right]\right]$ \\
\hline & & & $72\left[\left[\begin{array}{lll}2 & 1 & 1 \\
2 & 1 & 1\end{array}\right]\right] 144\left[\left[\begin{array}{lll}2 & 1 & 1 \\
1 & 2 & 1\end{array}\right]\right]$ & $\begin{array}{l}144\left[\left[\begin{array}{llll}2 & 1 & 1 & -\end{array}\right]\right] 144\left[\left[\begin{array}{llll}2 & 1 & 1 & - \\
1 & 1 & -1 & -1\end{array}\right]\right] \\
144\left[\left[\begin{array}{llll}2 & 1 & 1 & - \\
- & 2 & 1 & 1\end{array}\right]\right] 144\left[\left[\begin{array}{llll}2 & 1 & 1 & - \\
1 & 1 & -2\end{array}\right]\right] \\
72\left[\left[\begin{array}{llll}2 & 1 & 1 & - \\
- & 1 & 1 & 2\end{array}\right]\right]\end{array}$ \\
\hline
\end{tabular}




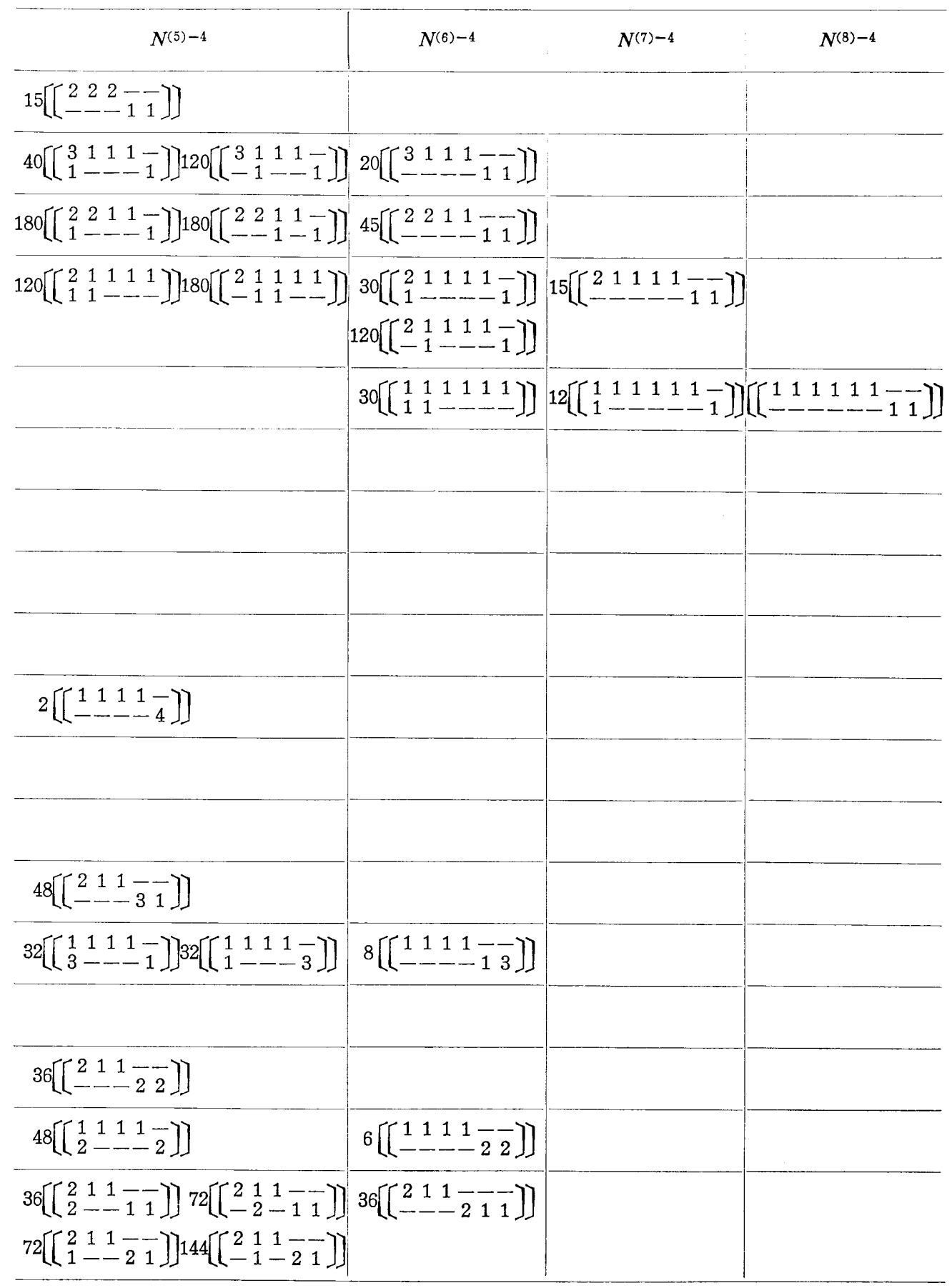


Table 3.1.3-6 Continue

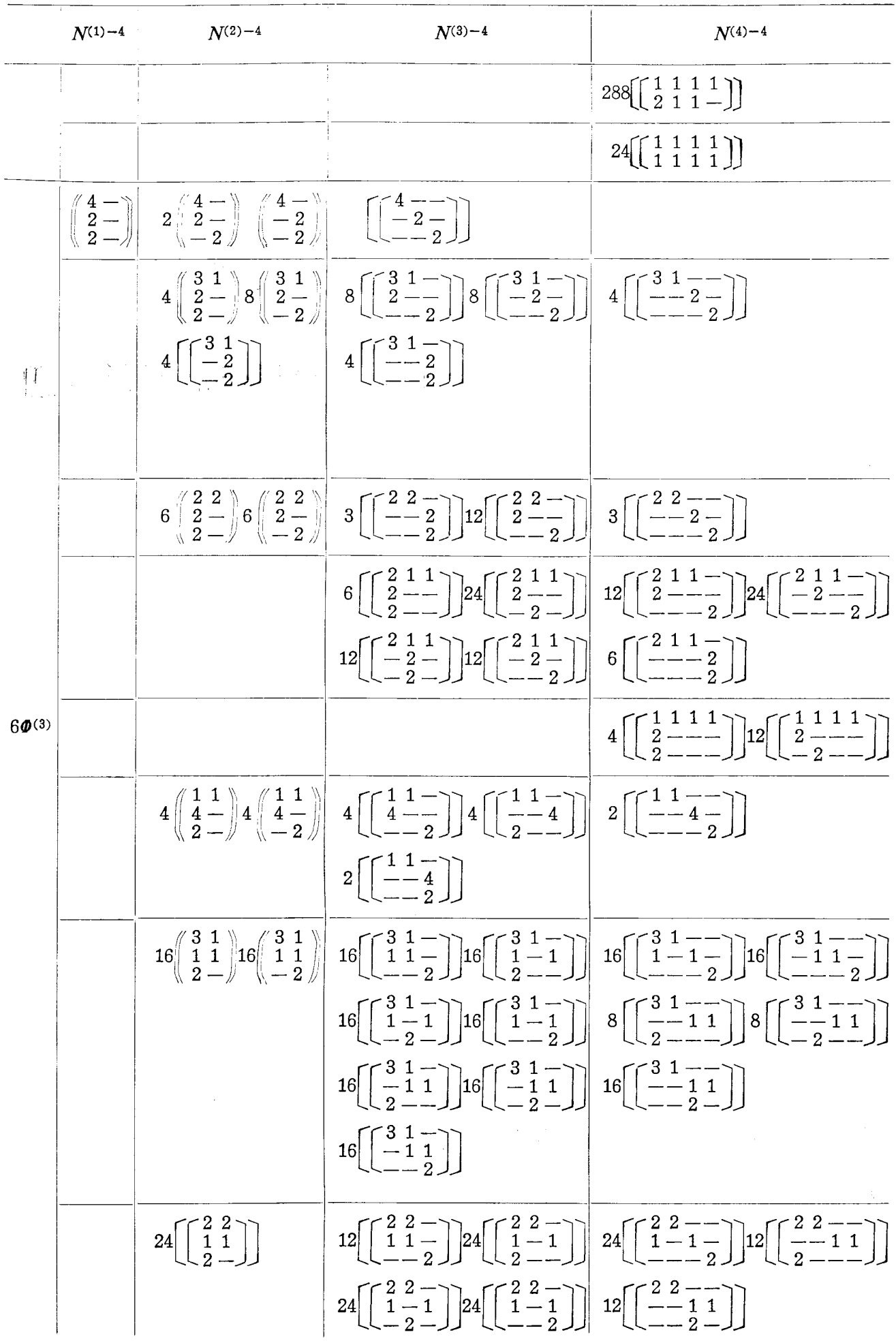




\begin{tabular}{|c|c|c|}
\hline$N^{(5)-4}$ & $N^{(6)-4}$ & $N^{(8)-4}$ \\
\hline $144\left[\left[\begin{array}{lllll}1 & 1 & 1 & 1 & - \\
1 & 1 & - & - & 2\end{array}\right]\right] 288\left[\left[\begin{array}{lllll}1 & 1 & 1 & 1 & - \\
2 & 1 & - & -1\end{array}\right]\right]$ & \begin{tabular}{l}
$48\left[\left[\begin{array}{llllll}1 & 1 & 1 & 1 & - & -\end{array}\right]\right] 12\left[\left[\begin{array}{lllllll}1 & 1 & 1 & 1 & - & - & -\end{array}\right]\right]$ \\
\hdashline $6\left[\left[\begin{array}{llllll}1 & 1 & 1 & 1 & - & -\end{array}\right]\right]$
\end{tabular} & \\
\hline $96\left[\left[\begin{array}{lllll}1 & 1 & 1 & 1 & - \\
1 & 1 & 1 & - & -1\end{array}\right]\right]$ & $72\left[\left[\begin{array}{llllll}1 & 1 & 1 & 1 & - & - \\
1 & 1 & - & - & 1 & 1\end{array}\right]\right] 16\left[\left[\begin{array}{lllllll}1 & 1 & 1 & 1 & - & - & -\end{array}\right]\right]$ & $111 \frac{1}{1}-\overline{1}-\overline{1}$ \\
\hline
\end{tabular}

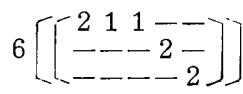

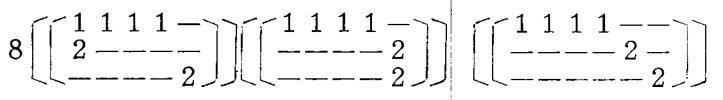

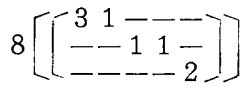

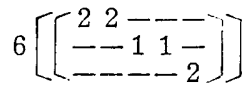


Table 3.1.3-7 Continue

$$
\begin{aligned}
& N^{(1)-1} \quad N^{(2)-4} \\
& N^{(3)-4} \\
& N^{(4)-4} \\
& 48\left[\left[\begin{array}{lll}
2 & 1 & 1 \\
1 & 1 & -
\end{array}\right] 48\left[\left[\begin{array}{lll}
2 & 1 & 1 \\
1 & 1 & - \\
- & 2 & -
\end{array}\right]\right] 48\left[\left[\begin{array}{llll}
2 & 1 & 1 & - \\
1 & 1 & - & -
\end{array}\right]\right] 24\left[\left[\begin{array}{llll}
2 & 1 & 1 & - \\
- & 1 & 1 & \overline{1}
\end{array}\right]\right]\right. \\
& 48\left[\left(\begin{array}{lll}
2 & 1 & 1 \\
1 & 1 & \frac{1}{2}
\end{array}\right]\right) \\
& 24\left[\left[\begin{array}{llll}
2 & 1 & 1 & - \\
1 & - & - & -1 \\
2 & - & - & -
\end{array}\right]\right] 48\left[\left[\begin{array}{cccc}
2 & 1 & 1 & - \\
- & 1 & - & -1 \\
2 & - & - & -
\end{array}\right]\right] \\
& \left.48\left[\left[\begin{array}{lll}
2 & 1 & 1 \\
- & 1 & 1 \\
- & - & 2
\end{array}\right]\right)\right] \\
& 48\left[\left[\begin{array}{llll}
2 & 1 & 1 & - \\
1 & - & - & 1 \\
- & 2 & -
\end{array}\right]\right] \\
& 24\left[\left[\begin{array}{lll}
2 & 1 & 1 \\
-2 & 1 & 1
\end{array}\right]\right]
\end{aligned}
$$

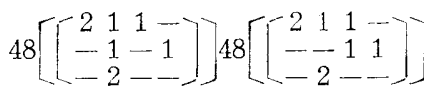

$$
\begin{aligned}
& 24\left[\left[\begin{array}{llll}
2 & 1 & 1 & - \\
1 & - & - & 1 \\
- & - & - & 2
\end{array}\right]\right] 48\left[\left[\begin{array}{cccc}
2 & 1 & 1 & - \\
- & 1 & - & 1 \\
- & - & - & 2
\end{array}\right]\right] \\
& 48\left[\left[\begin{array}{llll}
1 & 1 & 1 & 1 \\
1 & 1 & - & -
\end{array}\right]\right] 48\left[\left[\begin{array}{llll}
1 & 1 & 1 & 1 \\
1 & 1 & \frac{1}{2} \\
- & - & 2 & -
\end{array}\right]\right]
\end{aligned}
$$

$6 \Phi^{(3)}$

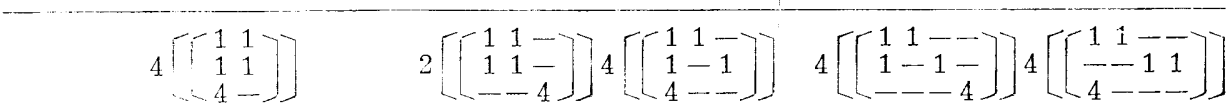

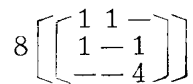

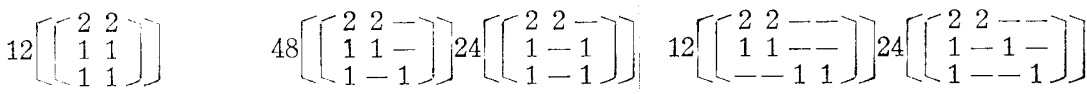

$$
\begin{aligned}
& 24\left[\left[\begin{array}{lll}
2 & 2 & - \\
1 & -1 & 1 \\
- & 1 & 1
\end{array}\right]\right]
\end{aligned}
$$

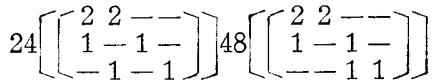

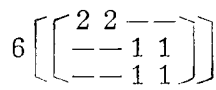




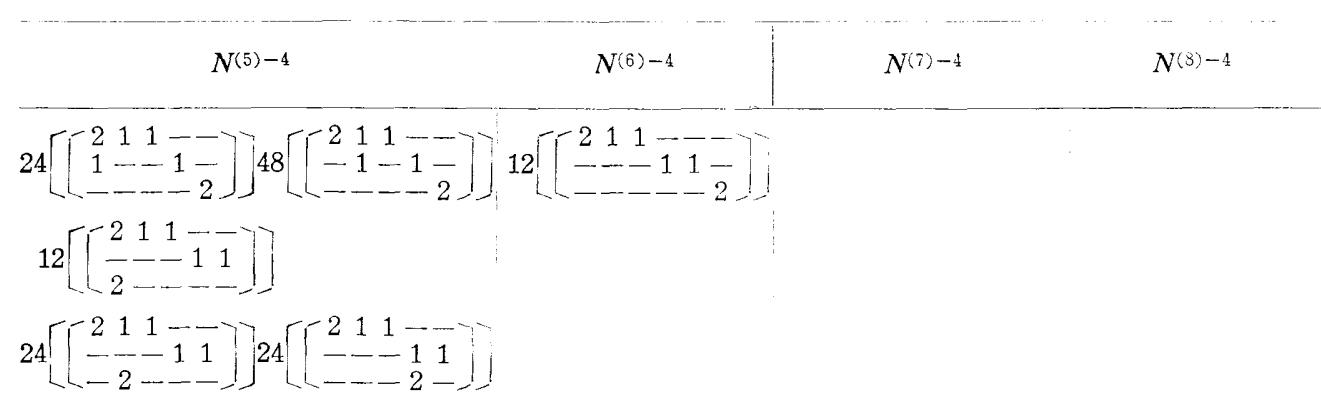

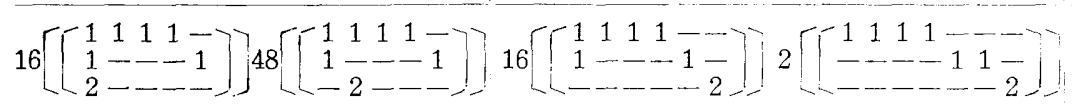

$$
\begin{aligned}
& 16\left[\left[\begin{array}{lllll}
1 & 1 & 1 & 1 & - \\
1 & - & - & - & 1 \\
& - & - & - & 2
\end{array}\right]\right] \\
& 8\left[\left[\begin{array}{llllll}
1 & 1 & 1 & 1 & - & 1 \\
\hdashline & - & - & - & 1 & 1
\end{array}\right]\right) \\
& 24\left[\left[\begin{array}{lllll}
1 & 1 & 1 & 1 & - \\
1 & 1 & - & - & - \\
- & - & - & -2
\end{array}\right]\right] \\
& 4\left[\left[\begin{array}{llllll}
1 & 1 & 1 & 1 & - & - \\
- & - & - & - & 1 & 1 \\
- & - & - & -2 & -
\end{array}\right]\right]
\end{aligned}
$$

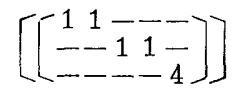

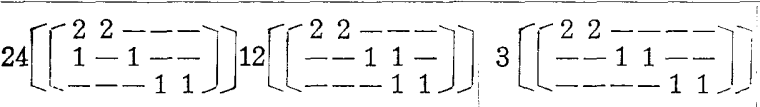


Table 3.1.3-8 Continue

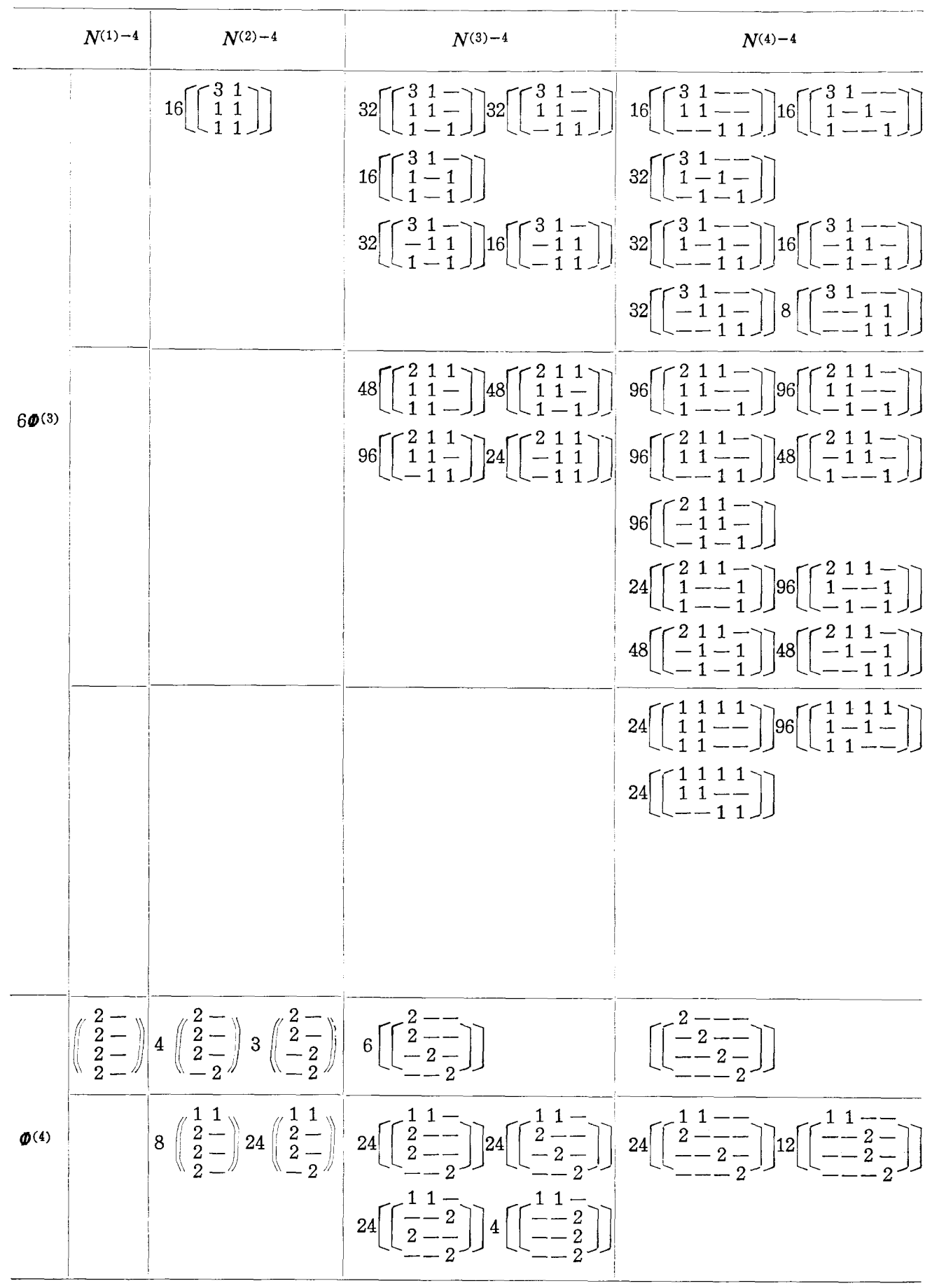




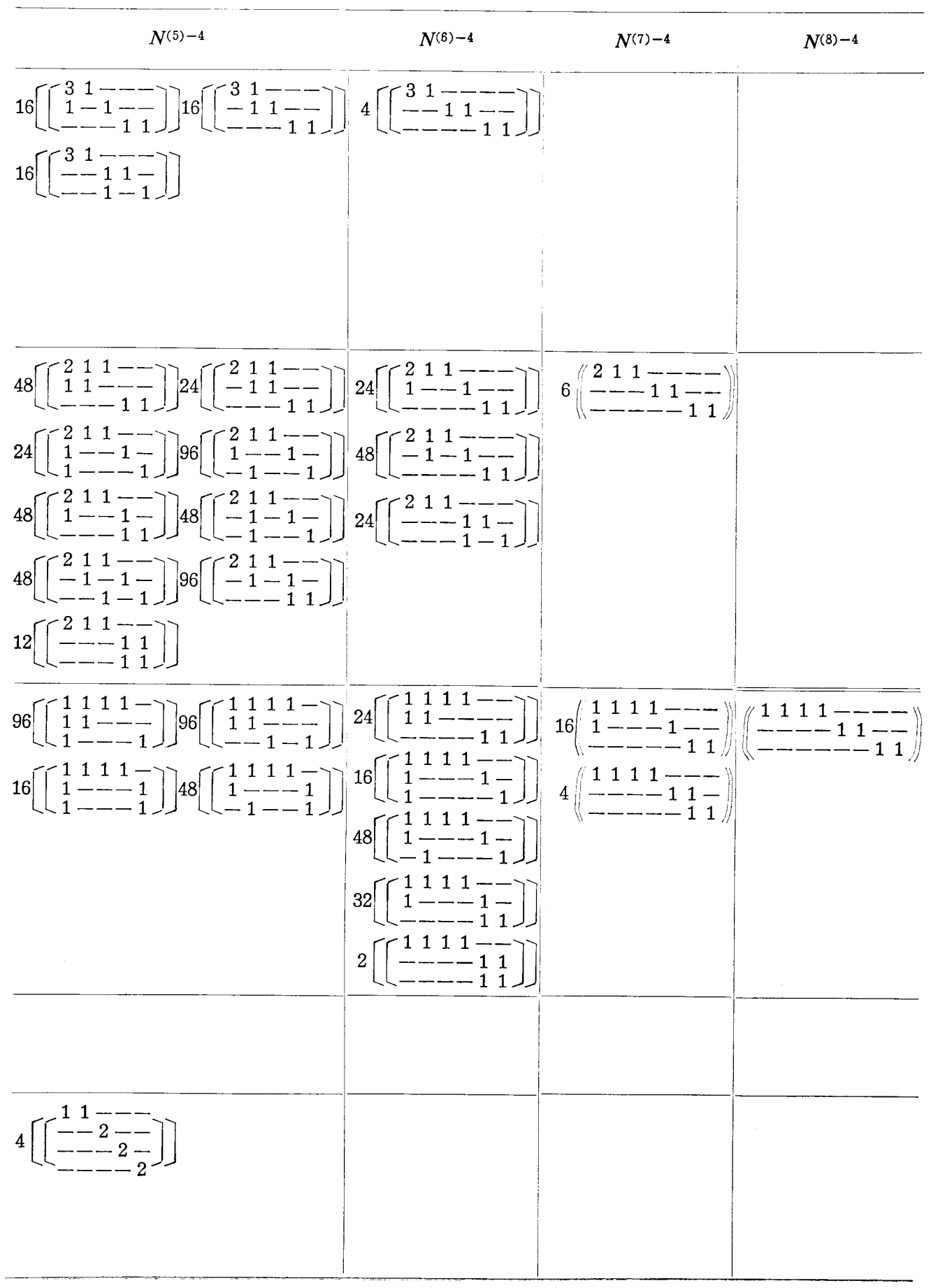


Table 3.1.3-9 Continue

$$
\begin{aligned}
& \begin{array}{llll}
N^{(1)-4} & N^{(2)-4} & N^{(3)-4} & N^{(4)-4}
\end{array}
\end{aligned}
$$

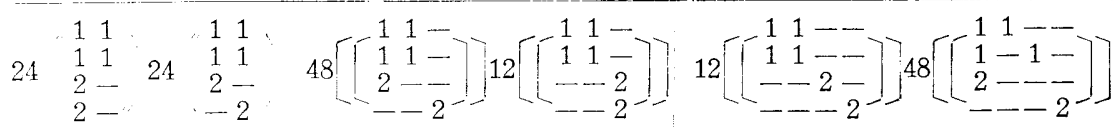

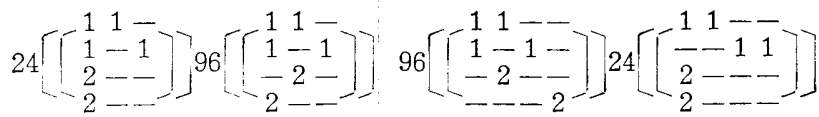

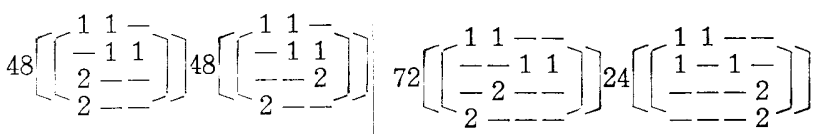

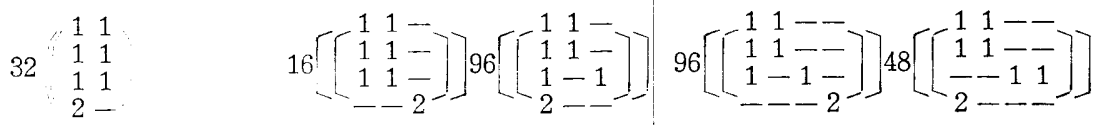

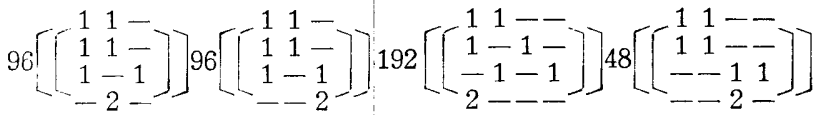

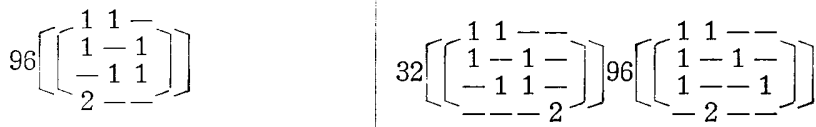

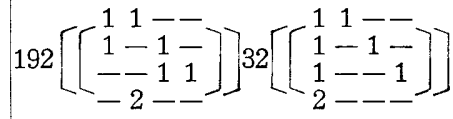

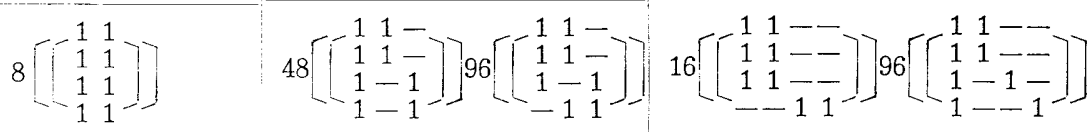

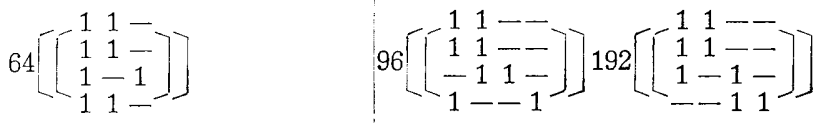

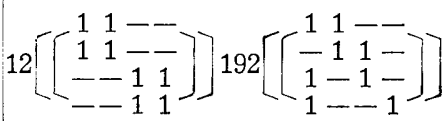

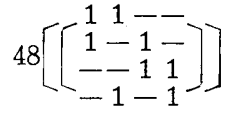




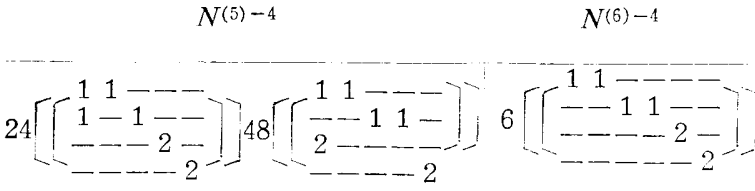

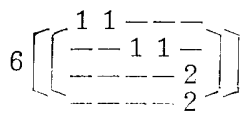

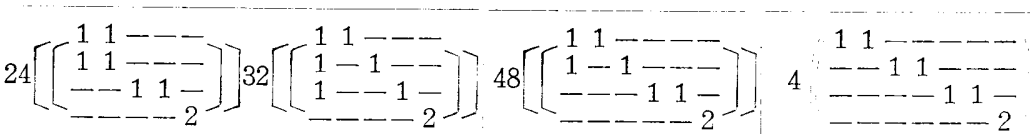

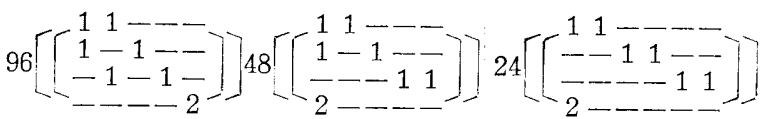

$$
\begin{aligned}
& 192\left[\left[\frac{1-1-\overline{1}}{-\frac{1}{-1}}\right]\right]
\end{aligned}
$$

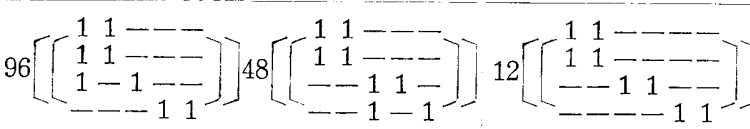

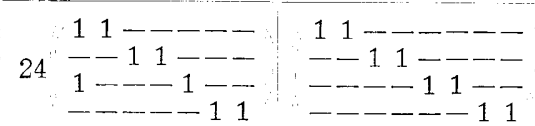

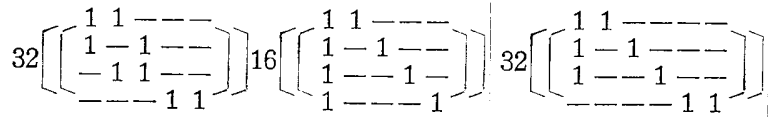

$192\left[\left[\begin{array}{l}1 \\ 1-1-\overline{-} \\ 1-1-1 \overline{1}\end{array}\right]\right]$

$96\left[\left[\frac{1}{1 \frac{1-\cdots}{1-}-\overline{1}}\right]\right]$

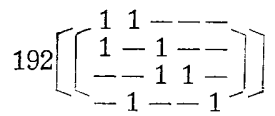

$98\left[\left[\begin{array}{c}1+1-\overline{1}-\overline{-} \\ ---1 \\ ---1-1\end{array}\right]\right.$ 


\subsection{Approximation of the distribution of $\lambda_{1 p}$ and $\lambda_{2}$}

\subsubsection{Moments of $\lambda_{1 p}$ and non-central chi-square distribution}

Let us calculate the higher moments of $\lambda_{1 p}$. The $S$-th moments of $\lambda_{1 p}$ can be represented in the following formula, such that,

$$
\operatorname{aver}\left\{\lambda_{1 p}^{S}\right\}=\left(\frac{1}{2}\right)^{S} \sum_{r=0}^{2 S}\left(\begin{array}{c}
2 S \\
r
\end{array}\right) N^{S-\frac{r}{2}} \alpha_{p}^{2 S-r} \operatorname{aver}\left\{\left(1_{p}\right)^{r}\right\}
$$

as we can see in the formula (3.1.4-1) (3.1.4-2) (3.1.4-3) and (3.1.4-4) for the 1st, 2nd, 3rd and 4 th moments, respectively.

Since, for odd degree s. m., we have

$$
\left\langle a_{p} b_{p} \cdots e_{p}\right\rangle^{*}=0 \text {, }
$$

then we can transform the $S$-th moments of $\lambda_{1 p}$,

$$
\operatorname{aver}\left\{\lambda_{1 p}^{S}\right\} \rightleftharpoons\left(\frac{1}{2}\right)^{S} \sum_{s=0}^{S}\left(\begin{array}{l}
2 S \\
2 s
\end{array}\right) N^{s-s} \alpha_{p}^{2 S-2 s} S_{s}
$$

and

$$
S_{s}=\sum_{h=1}^{2 s} N^{(h)-s} S_{s h},
$$

\begin{tabular}{|c|c|c|c|c|c|c|c|c|}
\hline $\begin{array}{l}\text { row } \\
\text { No. }\end{array}$ & (1) & (2) & (3) & (4) & (5) & (6) & (7) & (8) \\
\hline 1 & $\left|\begin{array}{l}N^{1-1} \\
\langle 2 p 》\end{array}\right|$ & $\mid \begin{array}{l}N^{(2)-1} \\
\ll 1^{2} \gg\end{array}$ & & & & & & \\
\hline 2 & $\begin{array}{l}N^{1-2} \\
\ll 4_{p} 》\end{array}$ & $\begin{array}{l}N^{(2)-2} \\
\left.4 \ll 3_{p} 1_{p}\right\rangle \\
\left.3 \ll 2_{p}^{2}\right\rangle\end{array}$ & $\begin{array}{c}N^{(3)-2} \\
6 \ll 2{ }^{2} 1_{p}^{2} 》\end{array}$ & $\begin{array}{c}N^{(4)-2} \\
\ll 1_{p}^{4} 》\end{array}$ & & & & \\
\hline 3 & $\begin{array}{l}N^{1-3} \\
\langle 6-\bar{p}\rangle\end{array}$ & $\begin{array}{l}N^{(2)-3} \\
6 \ll 5_{p} 1_{p} 》 \\
15 \ll 4_{p} 2_{p} 》 \\
\left.10 \ll 3_{p}^{2}\right\rangle\end{array}$ & $\begin{array}{l}N^{(3)-3} \\
15 \ll 4_{p} 1_{p}^{2} 》 \\
60 \ll 3_{p} 2_{p} 1_{p} 》 \\
15 \ll 2_{p}^{2} 》\end{array}$ & $\begin{array}{c}N^{(4)-3} \\
20 \ll 3{ }^{2} 1^{3} 》 \\
45 \ll 2_{p}^{2} 1_{p}^{2} 》\end{array}$ & $\begin{array}{l}N^{(5)-3} \\
15 \ll 2{ }_{p} 1_{p}^{4} 》\end{array}$ & $\begin{array}{l}N^{(6)-3} \\
\left\langle 1_{p}^{6} 》\right.\end{array}$ & & \\
\hline 4 & $\mid \begin{array}{l}N^{1-4} \\
\ll 8_{p} 》\end{array}$ & \begin{tabular}{|l|}
$N^{(2)-4}$ \\
$8 \ll 7_{p} 1_{p} 》$ \\
$28 \ll 6_{p} 2_{p} 》$ \\
$56 \ll 5_{p} 3_{p} 》$ \\
$\left.35 \ll 4_{p}{ }^{2}\right\rangle$
\end{tabular} & $\begin{array}{c}N^{(3)-4} \\
\left.28 \ll 6_{p} 1_{p}^{2}\right\rangle \\
\left.168 \ll 5_{p} 2_{p} 1_{p}\right\rangle \\
\left.280 \ll 3_{p} 4_{p} 1_{p}\right\rangle \\
\left.210 \ll 4_{p} 2_{p}^{2}\right\rangle \\
\left.280 \ll 3_{p} 2_{p}\right\rangle\end{array}$ & $\begin{array}{l}N^{(4)-4} \\
56 \ll 5_{p} 1_{p} 》 \\
420 \ll 4_{p} 2_{p} 1_{p}^{2} 》 \\
280 \ll 3_{p}^{2} 1_{p}^{2} 》 \\
840 \ll 3_{p} 2_{p}^{2} 1_{p} 》 \\
\left.105 \ll 2_{p}^{4}\right\rangle\end{array}$ & $\mid \begin{array}{c}N^{(5)-4} \\
70 \ll 4_{p} 1_{p}^{4} 》 \\
560 \ll 3_{p} 2_{p} 1_{p}^{3} 》 \\
420\left\langle 2_{p}^{3} 1_{p}^{2} 》\right.\end{array}$ & $\begin{array}{c}N^{(6)-4} \\
\left.56 \ll 3_{p} 1_{p}^{5}\right\rangle \\
\left.210 \ll 2_{p} 1_{p}^{4}\right\rangle\end{array}$ & $\mid \begin{array}{c}N^{(7)-4} \\
28 \ll 2_{p} 1_{p}{ }^{6} 》\end{array}$ & $\begin{array}{l}N^{(8)-4} \\
\ll 1_{p}^{8} 》\end{array}$ \\
\hline
\end{tabular}

where $S_{s h}$ means the sum of $2 s$-th degree s. m.'s having $h$ entries in $s$-th row in the following table.

Table 3.2.1 Representation of symmetric means in formula (3.2.3) 
In the above s. m. table, the s. m.'s can be easily calculated as

$$
\left.\left\langle a_{p} b_{p} \cdots e_{p}\right\rangle\right\rangle=\left\langle a_{p} b_{p} \cdots e_{p}\right\rangle *\langle a b \cdots e\rangle^{* * *},
$$

refering s. m.'s of $v_{n}$ 's and O.A. Then we have

$$
\begin{aligned}
& \left\langle 1_{p} 1_{p}\right\rangle=o\left(N^{-2}\right) \quad\left\langle\left\langle 3_{p} 1_{p}\right\rangle=o\left(N^{-2}\right)\right. \\
& \left\langle\left\langle 2_{p} 1_{p} 1_{p}\right\rangle=o\left(N^{-2}\right) \quad\left\langle\left\langle 1_{p}^{4}\right\rangle=o\left(N^{-4}\right)\right.\right. \\
& \left\langle 5_{p} 1_{p}\right\rangle=o\left(N^{-2}\right) \quad\left\langle\left\langle 4_{p} 1_{p} 1_{p}\right\rangle=o\left(N^{-2}\right)\right. \\
& \left\langle\left\langle 3_{p} 3_{p}\right\rangle\right\rangle=o\left(N^{-1}\right) \quad\left\langle\left\langle 3_{p} 2_{p} 1_{p}\right\rangle==o\left(N^{-2}\right)\right. \\
& \left\langle 3_{p} 1_{p}^{3}\right\rangle=o\left(N^{-4}\right) \quad\left\langle 2_{p}^{2} 1_{p}^{2}\right\rangle=o\left(N^{-2}\right) \\
& \left\langle\left\langle 2_{p} 1_{p}^{4}\right\rangle=o\left(N^{-4}\right) \quad\left\langle\left\langle 1_{p}^{6}\right\rangle=o\left(N^{-6}\right)\right.\right. \\
& \left\langle 7_{p} 1_{p}\right\rangle=o\left(N^{-2}\right) \quad\left\langle\left\langle 6_{p} 1_{p}^{2}\right\rangle=o\left(N^{-2}\right)\right. \\
& \left\langle\left\langle 5_{p} 3_{p}\right\rangle=o\left(N^{-1}\right) \quad\left\langle\left\langle 5_{p} 2_{p} 1_{p}\right\rangle=o\left(N^{-2}\right)\right.\right. \\
& \left\langle 5_{p} 1_{p} 1_{p} 1_{p}\right\rangle=o\left(N^{-4}\right) \quad\left\langle 4_{p} 3_{p} 1_{p}\right\rangle=o\left(N^{-2}\right) \\
& \left\langle 4_{p} 2_{p} 1_{p}^{2}\right\rangle=o\left(N^{-2}\right) \quad\left\langle\left\langle 4_{p} 1_{p}^{4}\right\rangle=o\left(N^{-4}\right)\right. \\
& \left\langle 3_{p}^{2} 2_{p}\right\rangle=o\left(N^{-1}\right) \quad\left\langle 33_{p}^{2} 1_{p}^{2}\right\rangle=o\left(N^{-2}\right) \\
& \left\langle\left\langle 3_{p} 2_{p}^{2} 1_{p}\right\rangle=o\left(N^{-4}\right) \quad\left\langle\left\langle 3_{p} 2_{p} 1_{p}^{3}\right\rangle=o\left(N^{-4}\right)\right.\right. \\
& \left\langle 3_{p} 1_{p}^{5}\right\rangle=o\left(N^{-6}\right) \quad\left\langle 2_{p}^{3} 1_{p}^{2}\right\rangle=o\left(N^{-2}\right) \\
& \left\langle 2_{p}^{2} 1_{p}^{4}\right\rangle=o\left(N^{-4}\right) \quad\left\langle\left\langle 2_{p} 1_{p}^{6}\right\rangle=o\left(N^{-6}\right)\right. \\
& \left\langle 1_{p}^{8}\right\rangle=o\left(N^{-8}\right) \text {. }
\end{aligned}
$$

If we denote the s. m.'s, containing odd entries, as $\langle\{$ odd ent $\}\rangle$ and 《\{odd ent $\} 》$, we have from formulas (2.4.10),

$$
\begin{aligned}
\ll\{\text { odd ent }\} \gg & =<\{\text { odd ent }\}>^{*}<\{\text { odd ent }\}>^{* *} \\
& =o\left(N^{-1}\right),
\end{aligned}
$$

and we have, for the all even entries s. m.'s

$$
\begin{aligned}
\left\langle 2_{p}\right\rangle & =\mu_{2} \\
\left\langle 4_{p}\right\rangle & =\mu_{4} \\
\left\langle 2_{p} 2_{p}\right\rangle & =\frac{N^{2}}{N^{(2)}} \mu_{2}^{2}+o\left(N^{-1}\right) \\
\left.\left\langle 6_{p}\right\rangle\right\rangle & =\mu_{6} \\
\left\langle 4_{p} 2_{p}\right\rangle & =\frac{N^{2}}{N^{(2)}} \mu_{4} \mu_{2}+o\left(N^{-1}\right) \\
\left\langle 2_{p} 2_{p} 2_{p}\right\rangle & =\frac{N^{3}}{N^{(3)}} \mu_{2}^{3}+o\left(N^{-1}\right) \\
\left.\left\langle 8_{p}\right\rangle\right\rangle & =\mu_{8} \\
\left\langle 6_{p} 2_{p}\right\rangle & =\frac{N^{2}}{N^{(2)}} \mu_{6} \mu_{2}+o\left(N^{-1}\right)
\end{aligned}
$$




$$
\begin{gathered}
\left\langle 4_{p} 4_{p}\right\rangle=\frac{N^{2}}{N^{(2)} \mu_{4}^{2}+o\left(N^{-1}\right)} \\
\left\langle 4_{p} 2_{p} 2_{p}\right\rangle=\frac{N^{3}}{N^{(3)}} \mu_{4} \mu_{2}^{2}+o\left(N^{-1}\right) \\
\left\langle 2_{p} 2_{p} 2_{p} 2_{p}\right\rangle=\frac{N^{4}}{N^{(4)}} \mu_{2}^{4}+o\left(N^{-1}\right) .
\end{gathered}
$$

Consequently, we get

$$
\operatorname{aver}\left\{\lambda_{1 p}\right\}=\left(\frac{1}{2}\right)\left\{N \alpha_{p}^{2}+\mu_{2}\right\}+o\left(N^{-1}\right) \text {. }
$$

If we put

$$
\operatorname{aver}\left\{\lambda_{1 p}\right\} \stackrel{d}{=} \underset{2}{\mu_{2}}\left\{2 \tau_{1 p}+1\right\}+o\left(N^{-1}\right),
$$

we get

$$
\tau_{1 p}=N \frac{\alpha_{p}^{2}}{2 \mu_{2}}+o\left(N^{-1}\right)
$$

In the case the $o\left(N^{-1}\right)$ can be neglected, we have

$$
\alpha_{p}^{2}=2 \tau_{1 p} \mu_{2} N^{-1} \text {. }
$$

Substituting this result to the formula (3.2.3), we get

\begin{tabular}{|c|c|c|c|c|c|c|c|c|}
\hline & 1 & 2 & 3 & 4 & 5 & 6 & 7 & 8 \\
\hline$s=1$ & 0 & -2 & & & & & & \\
\hline$s=2$ & 0 & $0, \quad-1$ & -2 & -4 & & & & \\
\hline$s=3$ & 0 & $0,-1,-1$ & -2 & $-2,-4$ & -4 & -6 & & \\
\hline$s=4$ & 0 & $0,-1,-2$ & $0,-1,-2$ & $\frac{0,-2,-3}{-4}$ & $-2, \quad-4$ & $-4,-6$ & -6 & -8 \\
\hline
\end{tabular}

$$
\operatorname{aver}\left\{\lambda_{1 p}^{S}\right\}=\left(\frac{1}{2}\right) \sum_{s=0}^{S}\left(\begin{array}{l}
2 S \\
2 s
\end{array}\right)\left(2 \tau_{1 p} \mu_{2}\right)^{s-s} S_{s} \text {. }
$$

So as to visualize the order of $N$ of $S_{s h}$ 's which are the elements of $S_{s}$, we shall tabulate the order of $N$ of $S_{s h}$ in the Table 3.2.1-1, and for the term of $N^{(h)-s}$ in the Table 3.2.1-2.

Furthermore, for the terms which contain the higher order term than

Table 3.2.1-1 Order of $N$ in the s. m.'s 
Table 3.2.2-2 Order of $N$ in $N^{(h)-s}$

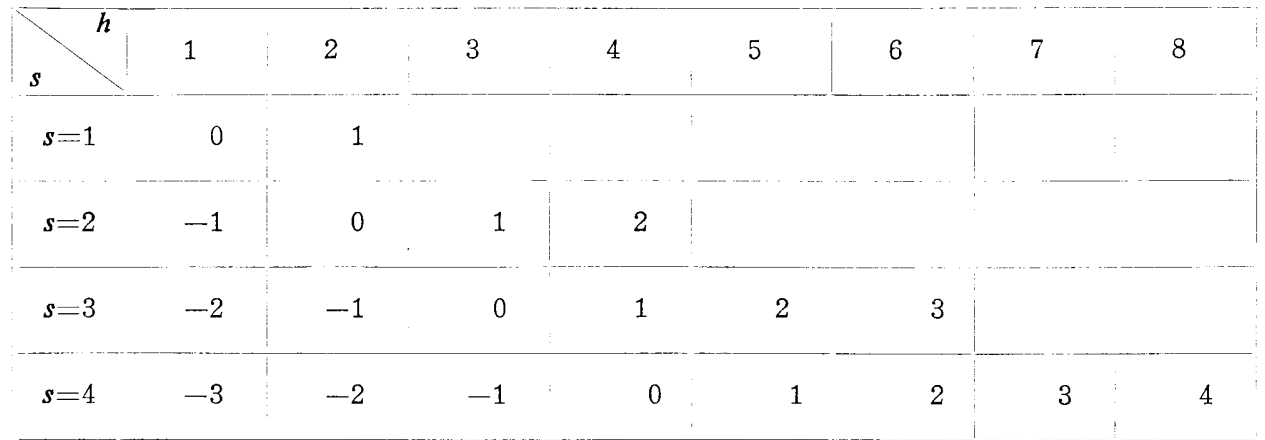

$N^{-1}$, we have

$$
\begin{aligned}
& N^{1-1}\left\langle 2_{p}\right\rangle=\mu_{2} \\
& \left.N^{(2)-1}\left\langle 1_{p}^{2}\right\rangle\right\rangle=\frac{1}{N-1} \mu_{2} \\
& N^{1-2}\left\langle 4_{p}\right\rangle=\frac{1}{N^{\mu_{4}}} \\
& N^{(2)-2} 3\left\langle 2_{p}^{2}\right\rangle=3\left(\mu_{2}^{2}-\frac{1}{N} \mu_{4}\right) \\
& N^{(3)-2} 6\left\langle 2_{p} 1_{p}^{2}\right\rangle=6 \mu_{2}^{2} \frac{1}{N-1}+o\left(N^{-2}\right) \\
& N^{(2)-3} 15\left\langle 4_{p} 2_{p}\right\rangle=\frac{15}{N^{\mu_{2} \mu_{4}}+o\left(N^{-2}\right)} \\
& \left.N^{(3)-3} 15\left\langle 2_{p}^{3}\right\rangle\right\rangle=15\left(\mu_{2}^{3}-3 \frac{1}{N^{-} \mu_{4} \mu_{2}}\right)+o\left(N^{-2}\right) \\
& \left.N^{(4)-3} 45 \ll 2_{p}^{2} 1_{p}^{2}\right\rangle=45 \frac{1}{N-1} \mu_{2}^{3}+o\left(N^{-2}\right) \\
& N^{(3)-4} 210\left\langle 4_{p} 2_{p}^{2}\right\rangle=210 \frac{1}{N} \mu_{4} \mu_{2}^{2}+o\left(N^{-2}\right) \\
& \left.N^{(4)-4} 105\left\langle 2_{p}^{4}\right\rangle\right\rangle=105\left(\mu_{2}^{4}-\frac{6}{\left.N^{-} \mu_{2}^{2} \mu_{4}\right)+o\left(N^{-2}\right)}\right. \\
& \left.N^{(5)-4} 420\left\langle 2_{p}^{3} 1_{p}^{2}\right\rangle\right\rangle=420 \mu_{2}^{4} \frac{1}{N-1}+o\left(N^{-2}\right) .
\end{aligned}
$$

In general, from the formulas $(2.3 .7),(2.4 .12)$ and $(2.4 .13)$, we have for the $2 s$-th degree $s, \mathrm{~m}$. 


$$
S_{s h}=o\left(N^{0}\right) \quad \text { for } h<s,
$$

and

$$
=o\left(N^{2(s-h)}\right) \quad \text { for } h>s \text {, }
$$

then we get

$$
\begin{aligned}
S_{s} & =\sum_{h=1}^{2 s} N^{(h)-s} S_{s h} \\
& =N^{(s)-s} S_{s s}+o\left(N^{-1}\right) .
\end{aligned}
$$

Furthermore, we have

$$
S_{s s}=C_{s}\left\langle\left\langle 2_{p}^{s}\right\rangle+o\left(N^{-1}\right),\right.
$$

and

$$
\left.\left\langle 2_{p}^{s}\right\rangle\right\rangle=N^{s-(s)} \mu_{2}^{s}+o\left(N^{-1}\right) .
$$

Then we get

$$
S_{s}=\frac{2 s}{(2 !)^{s} s !} \mu_{2}^{s}+o\left(N^{-1}\right) .
$$

Using these results, we easily get
(3.2.13-1)
$S_{1}=\mu_{2}+o\left(N^{-1}\right)$
(3.2.13-2)
$S_{2}=3 \mu_{2}^{2}+o\left(N^{-1}\right)$
(3.2.13-3)
$S_{3}=15 \mu_{2}^{3}+o\left(N^{-1}\right)$
$(3.2 .13-4)$
$S_{4}=105 \mu_{2}^{4}+o\left(N^{-1}\right)$

and

$$
S_{s}=1 \cdot 3 \cdot 5 \cdots(2 s-1) \mu_{2}^{s}+o\left(N^{-1}\right) .
$$

Finally, we get

THEOREM B-1: The moments of $\lambda_{1 p}$ are as follows

$$
\begin{aligned}
& \text { aver } \lambda_{1 p}=\frac{\mu_{2}}{2}\left\{2 \tau_{1 p}+1\right\}+\Delta_{1 p}+o\left(N^{-2}\right) \\
& \text { aver } \lambda_{1 p}^{2}=\left(\frac{\mu_{2}}{2}\right)^{2}\left\{4 \tau_{1 p}^{2}+12 \tau_{1 p}+3\right\}+\Delta_{2 p}+o\left(N^{-2}\right) \\
& \text { aver } \lambda_{1 p}^{3}=\left(\frac{\mu_{2}}{2}\right)^{3}\left\{8 \tau_{1 p}^{3}+60 \tau_{1 p}^{2}+90 \tau_{1 p}+15\right\}+\Delta_{3 p}+o\left(N^{-2}\right)
\end{aligned}
$$

and

$$
\text { aver } \begin{gathered}
\lambda_{1 p}^{4}=\left(\frac{\mu_{2}}{2}\right)^{4}\left\{16 \tau_{1 p}^{4}+224 \tau_{1 p}^{3}+840 \tau_{1 p}^{2}+840 \tau_{1 p}+105\right\} \\
+\Delta_{4 p}+o\left(N^{-2}\right)
\end{gathered}
$$

where

$(3.2 .15-1)$

$$
\tau_{1 p}=\frac{N \alpha_{p}^{2}}{2 \mu_{2}}
$$




$$
\begin{aligned}
& \Delta_{1 p}=N-1 \text { - }\left(\begin{array}{c}
\mu_{2} \\
2
\end{array}\right) \\
& \Delta_{2 p}=\left\{\begin{array}{c}
12 \tau_{1 p} \\
N-1
\end{array}-\frac{2}{N}\left(\begin{array}{c}
\mu_{4} \\
\mu_{2}^{2}
\end{array}\right)+\begin{array}{c}
6 \\
N-1
\end{array}\right\}\left(\begin{array}{c}
\mu_{2} \\
2
\end{array}\right)^{2} \\
& \Delta_{3 p}=\left\{\begin{array}{l}
60 \tau_{1 p}^{2} \\
N-1
\end{array}-\frac{180 \tau_{1 p}}{3 N}\left(\frac{\mu_{4}}{\mu_{2}^{2}}\right)+\frac{180 \tau_{1 p}}{N-1}\right. \\
& \left.-\frac{30}{N}\left(\frac{\mu_{4}}{\mu_{2}^{2}}\right)+\frac{45}{N-1}\right)\left(\begin{array}{c}
\mu_{2} \\
2
\end{array}\right)^{3} \\
& \Delta_{4 p}=\left[\begin{array}{l}
224 \tau_{1 p}^{3} \\
N-1
\end{array}-\left\{840 \tau_{1 p}^{2}\left(\frac{2}{3 N}\left(\frac{\mu_{4}}{\mu_{2}^{2}}\right)-\frac{2}{N-1}\right)\right.\right. \\
& -840 \tau_{1 p}\left(\frac{2}{N}\left(\frac{\mu_{4}}{\mu_{2}^{2}}\right)-\frac{3}{N-1}\right) \\
& \left.\left.-\left(\frac{420}{N}\left(\frac{\mu_{4}}{\mu_{2}^{2}}\right)-\frac{420}{N-1}\right)\right\}\right]\left(\begin{array}{c}
\mu_{2} \\
2
\end{array}\right)^{4} .
\end{aligned}
$$

In general, the $S$-th $(S<\infty)$ moment of $\lambda_{1 p}$ is

$$
\text { aver } \begin{aligned}
\lambda_{1 p}^{S}=\left(\frac{\mu_{2}}{2}\right)^{S} & \left\{\left(2 \tau_{1 p}\right)^{S}+\sum_{s=1}^{S} \prod_{r=1}^{s}(2 r-1)\left(\begin{array}{l}
2 S \\
2 s
\end{array}\right)\left(2 \tau_{1 p}\right)^{s-s}\right\} \\
+o\left(N^{-1}\right) & \text {. }
\end{aligned}
$$

On the other hand, we have the moment of

$$
X^{2}=(\sqrt{2} \tau+x)^{2}
$$

such that

$$
E\left\{(\sqrt[V]{ } / 2 \tau+x)^{2}\right\}^{s}=(2 \tau)^{s}+\sum_{s=1}^{s}\left(\begin{array}{l}
2 S \\
2 s
\end{array}\right)(2 \tau)^{s-s} E\left(x^{2 s}\right),
$$

where $x$ is normally and independently distributed variate with mean 0 and variance 1 . Then we get the moments of the non-central chi-square distribution with the degree of freedom 1 and the noncentral parameter $\tau$, and we have
$(3.2 .18-1)$
$E\left\{(\sqrt{2} \tau+x)^{2}\right\}=2 \tau+1$
$(3.2 .18-2)$
$E\left\{(\sqrt{2 \tau}+x)^{2}\right\}^{2}=4 \tau^{2}+12 \tau+3$
$(3.2 .18-3)$
$E\left\{(\sqrt{2 \tau}+x)^{2}\right\}^{3}=8 \tau^{3}+60 \tau^{2}+90 \tau+15$
$(3.2 .18-4)$
$E\left\{(\sqrt{2} \tau+x)^{2}\right\}^{4}=16 \tau^{4}+224 \tau^{3}+840 \tau^{2}+840 \tau+105$

and in general

$(3.2 .18-5)$

$$
\mu_{s}^{\prime}=(2 \tau)^{s}+\sum_{s=1}^{S} \prod_{r=1}^{S}(2 r-1)\left(\begin{array}{l}
2 S \\
2 s
\end{array}\right)(2 \tau)^{S-s}
$$

From these results, it can be seen that the distribution of $\left\{\lambda_{1 p} \cdot\left(\begin{array}{c}\mu_{2} \\ 2\end{array}\right)^{-1}\right\}$ 
is approximately the non-central chi-square distribution with the degree of freedom 1 and non-central parameter

$$
\tau_{p}=N_{2 \mu_{2}}^{\alpha_{p}^{2}}
$$

\subsubsection{Moments of $\lambda_{2}$ and central chi-squares}

Now, we proceed into the calculation of the moments of $\lambda_{2}$. These moments of $\lambda_{2}$ can be represented in the following formula, as we have seen in section 3.1.2.

$$
\begin{aligned}
& \operatorname{aver}\left\{\lambda_{2}^{K}\right\}=\left(\frac{1}{2}\right)^{K} \sum_{k=1}^{2 K} \text { aver } G_{k}, \\
& \operatorname{aver} G_{k}=\Sigma^{\prime}\left(\frac{N^{(k)}}{N^{K}}\right) \Phi^{(\varphi)} \quad[(\varphi \times h)]_{u}^{*}<\{h\}>^{* *} C_{u},
\end{aligned}
$$

where $\Sigma^{\prime}$ stands for the summation over the all g. s. m.'s with $h$ columns, and where $[(\varphi \times h)]^{*}{ }_{u}$ is $u$-th g. s. m. with $\varphi$ rows and $h$ columns of O. A. and $C_{u}$ is the constant of the $u$-th g. s. m. as we can see the Table 3.1.1., 3.1.2, 3.1.3 and 3.1.4.

If we denote the g. s. m. consisted of even entries as $[\{\underset{\text { even ent }}{\varphi} \times \boldsymbol{h}\}]^{*}$ and g. s. m. including odd entries as $[\underset{\text { odd ent }}{\{\varphi \times \bar{n}}\}]^{*}$ we have

$$
\begin{array}{c|c}
\text { for } h<K & \multicolumn{1}{c}{\text { for } K<h} \\
\left(\begin{array}{c}
N^{(h)} \\
N^{K}
\end{array}\right) & \left(\frac{N^{(h)}}{N^{K}}\right) \\
{\left[\left\{\varphi_{\text {even ent }} \times h\right\}\right]^{*}=1} & \\
[\underset{\text { odd ent }}{\{\varphi h}\}]^{*}=o\left(N^{\circ}\right) & {[\{\varphi \times h\}]^{*}=o\left(N^{-(h-K)}\right)} \\
<\{h\}>^{* *}=o\left(N^{\circ}\right) & <\{h\}>^{* *}=o\left(N^{-(h-K)}\right),
\end{array}
$$

from the results of symmetric means of $v_{n}$ 's and g. s. m.'s of O. A., then we get

$$
S_{h}=o\left(N^{-1}\right), \quad \text { for } h>K \text {. }
$$

Consequently, it can be obtained that

$$
\operatorname{aver}\left\{\lambda_{2}^{K}\right\}=\left(\frac{1}{2}\right) S_{K}+o\left(N^{-1}\right) \text {, }
$$

where

$$
\begin{aligned}
S_{K}= & S(\text { even })+S(\text { odd }), \\
& S(\text { even })=\text { Sum of symmetric means as } \\
& {[\{\text { even }\}]^{*} \text { 's consisting of all even entries. } } \\
& S(\text { odd })=\text { Sum of symmetric means as } \\
& {[\text { odd }\}]^{*} \text { 's in odd entries. } }
\end{aligned}
$$


As we have seen in the previous section for s. m.'s we also have at most the $K$ all 2 entries in the $2 K$-th degree g. s. m.'s which are containing the all even entries, such that

$$
\begin{aligned}
\left\langle\{K\}>^{* *}\right. & =\left\langle 2 \cdots 2>^{* * *}\right. \\
& =\frac{\left(N \mu_{2}\right)^{K}}{N^{(K)}}+o\left(N^{-1}\right) .
\end{aligned}
$$

On the contrary, since the all odd c. g. s. m.'s are containing at most $2 K$ all odd entries in the $2 K$-th degree g. s. m.'s, then we have no $\varphi(\geq 3) \times K$ g. s. f. having all odd entries

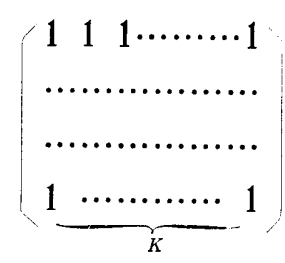

and containing no zero entry in the $2 K$-th degree g. s. m.'s.

Consequently refering the formula (2.2.20), we have [\{odd ent $\}]^{*}=O\left(N^{-1}\right)$. Since we have in the previous section

$$
<\{K\}>^{* *}=o\left(N^{\circ}\right)
$$

we get

$$
S(\text { odd })=o\left(N^{-1}\right)
$$

Then we get

$$
S^{K}=\mu_{2}^{K} \sum_{\varphi=1}^{K} \Phi^{(\varphi)} C_{\varphi}\left(<2^{K}>\right)^{* *}+o\left(N^{-1}\right),
$$

where $C_{\varphi}\left(<2^{K}>^{* *}\right)$ is the sum of numerical coefficients of g. s. m.'s with $\varphi$ rows and $K$ columns of which all entries equal to 2 . Furthermore, adding the calculations of the g. s. m.'s those order of $N^{-1}$, we finally get

Theorem B-2: The moments of $\lambda_{2}$ are

$$
\begin{aligned}
& \text { aver }\left\{\lambda_{2}\right\}=\left(\begin{array}{c}
\mu_{2} \\
2
\end{array}\right) \Phi+\Delta_{1}+o\left(N^{-2}\right) \\
& \text { aver }\left\{\lambda_{2}^{2}\right\}=\left(\frac{\mu_{2}}{2}\right)^{2}\left\{3 \Phi+\Phi^{(2)}\right\}+\Delta_{2}+o\left(N^{-2}\right)
\end{aligned}
$$$$
\operatorname{aver}\left\{\lambda_{2}^{3}\right\}=\left(\frac{\mu_{2}}{2}\right)^{3}\left\{15 \Phi+9 \Phi^{(2)}+\Phi^{(3)}\right\}+\Delta_{3}+o\left(N^{-2}\right)
$$

and

$$
\begin{gathered}
\text { aver }\left\{\lambda_{2}^{4}\right\}=\left(\begin{array}{c}
\mu_{2} \\
2
\end{array}\right)^{4}\left\{105 \Phi+(15 \times 4+9 \times 3) \Phi^{(2)}+6 \times 3 \Phi^{(3)}+\Phi^{(4)}\right\} \\
+\Delta_{4}+o\left(N^{-2}\right)
\end{gathered}
$$


where

$(5.2 .28-1)$

$$
\Delta_{1}=\frac{\mu_{2}}{2} \Phi \frac{1}{N-1}
$$

$$
\Delta_{2}=\left(\begin{array}{c}
\mu_{2} \\
2
\end{array}\right)^{2}\left\{\left(6-2 \delta_{2}\right) \Phi\right\} \frac{1}{N}
$$

$$
\Delta_{3}=\left(\frac{\mu_{2}}{2}\right)^{3}\left\{\left(-30 \delta_{2}+45\right) \Phi-3\left(2 \delta_{2}+3\right) \Phi^{(2)}+\left(-3+4 \delta_{1}\right) \Phi^{(3)}\right\} \frac{1}{N}
$$

$$
\Delta_{4}=\left(\frac{\mu_{2}}{2}\right)^{4}\left\{\left(-420 \delta_{2}+420\right) \Phi-\left(156 \delta_{2}+228\right) \Phi^{(2)}+\left(-2 \delta_{2}+24 \delta_{1}\right.\right.
$$

$$
-18) 6 \Phi^{(3)}+\left(16 \delta_{1}-8\right) \Phi^{(4)} ! \frac{1}{N},
$$

$(3.2 .29-1)$

$$
\delta_{1} \stackrel{d}{=} \frac{\mu_{3}^{2}}{\mu_{2}^{3}} r_{3}
$$

$$
\delta_{2} \stackrel{d}{=} \frac{\mu_{4}}{\mu_{2}^{2}}
$$

\section{In general}

$$
\operatorname{aver}\left\{\lambda_{2}^{K}\right\}=\left(\frac{\mu_{2}}{2}\right)^{K} \sum_{\varphi} C_{\varphi}\left\{<2^{K}>^{* *}\right\} \Phi^{(\varphi)}+o\left(N^{-1}\right)
$$

Proof: In the formula $(3.1 .12-1),(3.1 .12-2),(3.1 .12-3)$ and $(3.1 .12-4)$, the all even g. s. m.'s in the $K$ th column have the order of $N^{\prime}$. Furthermore in the $(K-1)$ th, $K$ th and $(K+1)$ th columns, we have the g. s. m.'s which have the order at most $N^{-1}$. These g. s. m.'s are as follows:

$$
[K=1]
$$

$$
\left[\left[\begin{array}{l}
2- \\
-
\end{array}\right]\right] N^{(1)-1}=\mu_{2}, \quad\left[\left[\begin{array}{cc}
1 & 1 \\
- & -
\end{array}\right]\right] N^{(2)-1}=\mu_{2} / N-1
$$

$[K=2]$

$$
\begin{aligned}
& {\left[\left[\begin{array}{c}
4- \\
--
\end{array}\right]\right] N^{(1)-2}=\mu_{4} / N,} \\
& {\left[\left[\begin{array}{ll}
2 & 2 \\
- & -
\end{array}\right]\right] N^{(2)-2}=\mu_{2}^{2}-\mu_{4} N^{-1}} \\
& {\left[\left[\begin{array}{l}
2- \\
2-
\end{array}\right]\right] N^{(1)-2}=\quad " \text {, }} \\
& {\left[\left[\frac{2-}{2}\right]\right] N^{(2)-2}=\quad \text { " }} \\
& {\left[\left[\begin{array}{ccc}
2 & 1 & 1 \\
- & - & -
\end{array}\right]\right] N^{(3)-2}=\mu_{2}^{2} / N-1 \text {, }} \\
& {\left[\left[\begin{array}{ll}
1 & 1 \\
1 & 1
\end{array}\right]\right] N^{(2)-2}=-\mu_{2}^{2} \frac{1}{N-1}}
\end{aligned}
$$

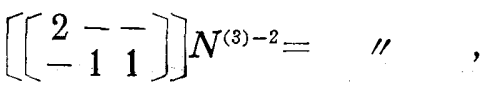

$[K=3]$ 


$$
\begin{aligned}
& {\left[\left[\begin{array}{ll}
4 & 2 \\
- & -
\end{array}\right]\right] N^{(2)-3}=\mu_{4} \mu_{2} N^{-1}+o\left(N^{-2}\right), \quad\left[\left[\begin{array}{lll}
2 & 2 & 2 \\
- & -
\end{array}\right]\right] N^{(3)-3}=\mu_{2}^{3}-3 \mu_{4} \mu_{2} N^{-1}+o\left(N^{-2}\right)}
\end{aligned}
$$

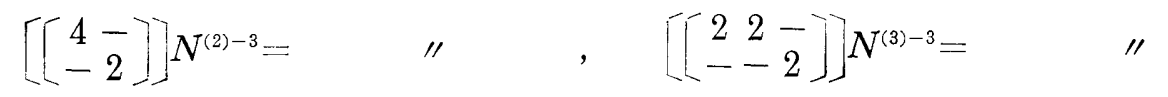

$$
\begin{aligned}
& {\left[\left[\begin{array}{ll}
2 & 2 \\
2 & -
\end{array}\right]\right] N^{(2)-3}=\quad \quad \text { " } \quad\left[\left[\begin{array}{c}
2--2- \\
--2
\end{array}\right]\right] N^{(3)-3}=\quad \text { " }} \\
& {\left[\left[\begin{array}{l}
2- \\
2- \\
-2
\end{array}\right] N^{(2)-3}=\quad \quad \quad \quad \quad\left[\left[\begin{array}{cccc}
2 & 2 & 1 & 1 \\
- & - & - & -
\end{array}\right]\right] N^{(4)-3}=\mu_{2}^{3}\left(\begin{array}{c}
1 \\
N-1
\end{array}\right)+o\left(N^{-2}\right)\right.} \\
& {\left[\left[\begin{array}{lll}
1 & 1 & 2 \\
1 & 1 & -
\end{array}\right]\right] N^{(3)-3}=-\mu_{2}^{3} \frac{1}{N-1}+o\left(N^{-2}\right),\left[\left[\begin{array}{cccc}
2 & 2 & - & -
\end{array}\right]\right] N^{(4)-3}=\quad \text { " }}
\end{aligned}
$$

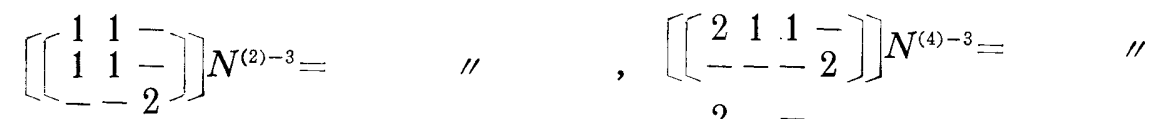

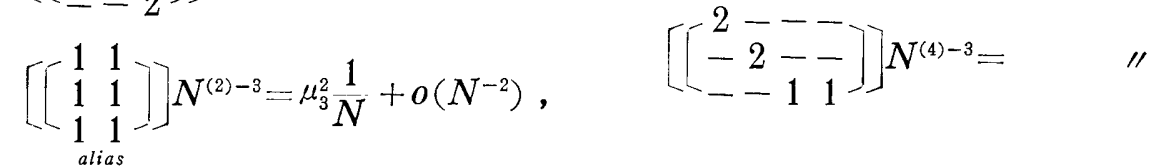

[K=4]

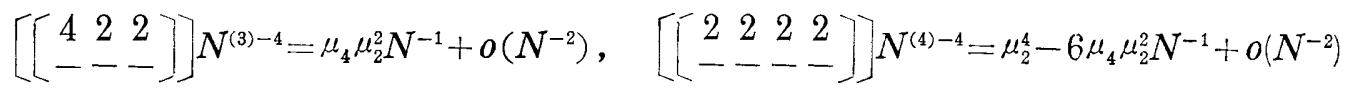

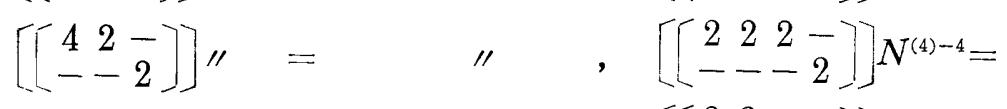

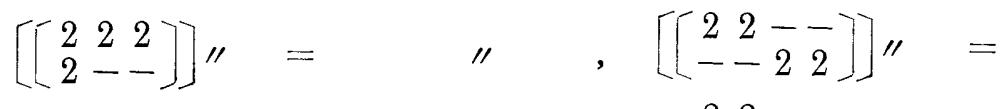

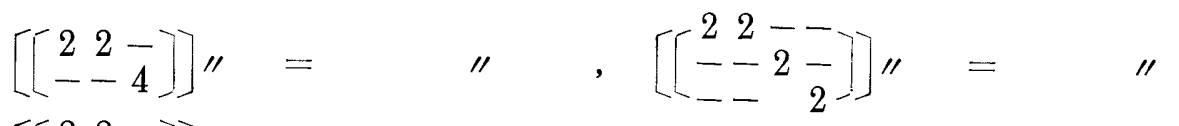

$$
\begin{aligned}
& {\left[\left[\begin{array}{lll}
2 & 2 & -\overline{2} \\
2 & - & 2
\end{array}\right] \prime \prime=\right.} \\
& {\left[\left[\begin{array}{c}
4-2- \\
-
\end{array}\right]\right]^{\prime \prime}=}
\end{aligned}
$$

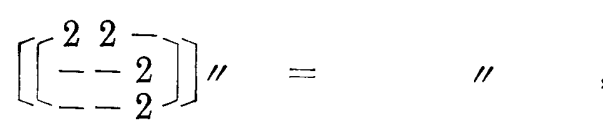

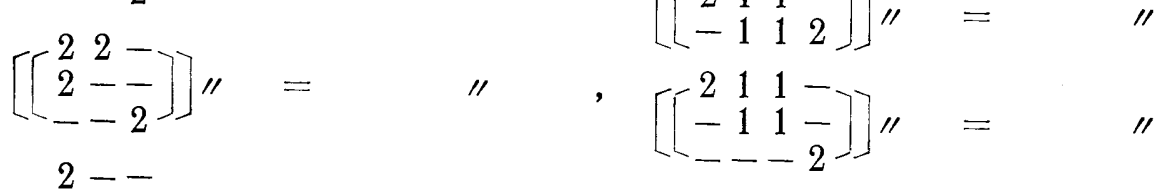

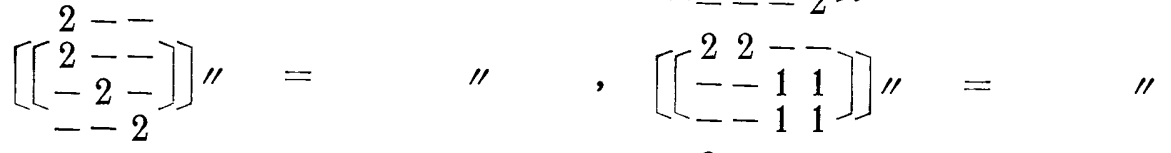

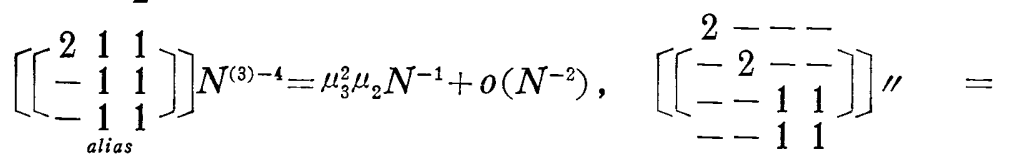

$$
\begin{aligned}
& {\left[\left[\begin{array}{llll}
2 & 2 & 1 & 1 \\
- & -1 & 1 & 1
\end{array}\right]\right]^{\prime \prime}=-\mu_{2}^{4} \frac{1}{N-1}+o\left(N^{-2}\right)} \\
& {\left[\left[\begin{array}{llll}
2 & 1 & 1 & - \\
- & 1 & 1 & 2
\end{array}\right]\right] \prime \prime=} \\
& {\left[\left[\begin{array}{c}
-2-- \\
--2 \frac{-}{2}
\end{array}\right]\right]^{\prime \prime}=} \\
& ---2
\end{aligned}
$$




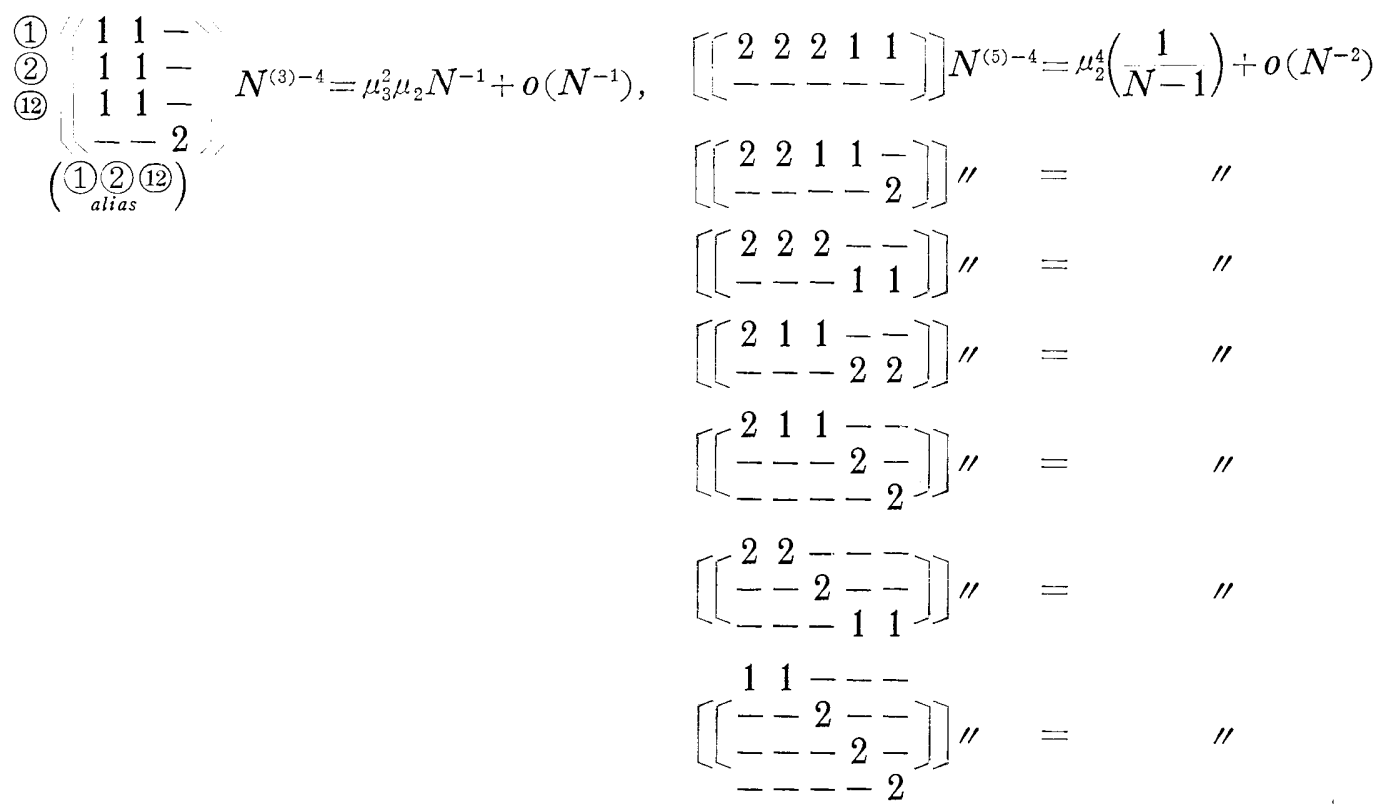

The other g. s. m.'s are the negligible terms those order are at most $N^{-2}$ by virtue of the results of chapter 2 in this paper.

For the moments of the central chi-square distribution with the degrees of freedom $\Phi$, we have

$$
E\left(\sum_{\phi=1}^{\infty} x_{\phi}^{2}\right)^{K}=\sum_{\phi=1}^{\infty} C_{\phi}\left(<2^{K}>^{* *}\right) \Phi^{(\phi)},
$$

where $x_{1}, x_{2}, \cdots, x_{\oplus-1}$ and $x_{\oplus}$ are the mutual independent normal variates with same mean 0 and same variance 1 , for example

$$
\begin{aligned}
& \mu_{1}^{\prime} \stackrel{d}{=} E\left(\sum_{\phi=1}^{\infty} x_{\phi}^{2}\right)=\Phi \\
& \mu_{2}^{\prime}=E\left\{\left(\sum_{\phi=1}^{\infty} x_{\phi}^{2}\right)^{2}\right\} \\
& =\Phi E\left(x_{\phi}^{4}\right) \div \Phi^{(2)} E\left(x_{\phi 1}^{2}\right) E\left(x_{\phi}^{2}\right)
\end{aligned}
$$

and so on. Consequently, we finally obtain the conclusion that the asymptotic distribution of $\left\{\lambda_{2}\left(\mu_{2} / 2\right)^{-1}\right\}$ is the central chi-square distribution with degrees of freedom $\Phi$, in the condition that the terms $\left\{\Delta_{K}\right\}(K=1,2, \cdots$, 4) are negligible order.

\section{[Acknowledgements]}

The auther expresses his gratitude to Professor Kitagawa for his helpful guidance of full course of this research and kind encouragements. His gratitude is also due to Dr. Shimada for his helpful suggestions and kind encouragements, and also due to many members of Kasado Works Hitachi Ltd. for practical applications of this RACOFFD method and carrying 


\section{through a large scale numerical computation.}

\section{References}

[1] Behnken, D. W.; Sampling moments of means from finite multivariate populations, Ann. Math. Statist. 32 (1961), 406-413.

[2] Box, G.E.P. and Hunter, J.S.; The $2^{K-P}$ fractional factorial designts Part I, Technometrics 3 (1961), 311-351.

[3] Box, G.E.P. and Hunter, J.S.; The $2^{K-P}$ fractional factorial designs Part II, Technometrics 3 (1961), 449-458.

[4] David, F.N. and Kendall, M.G.; Tables of symmetric functions Part I, Biometrika 36 (1949), 431-449.

[5] Fisher, R.A.; Moments and product moments of sampling distribution, London Mathematical Society, Series 2, 30 (1928), 199-238.

[6] Hooke, R.; Symmetric function of a two-way array, Ann. Math. Statist. 27 (1956), 55-79.

[7] Rosson, D.S.; Applications of multi-variate polykays to theory of unbiased ratio-type estimation, Jour. Amer. Statist. Assoc., 52 (1957), 511-522.

[8] Shimada, S.; Introduction to stochastics (1955) Denki-Shoin, (in Japanese).

[9] Turey, J.W.; Some sampling simplified, Jour. Amer. Statist. Assoc., 45 (1950), 501-519.

[10] Tukey, J.W.; Keeping moment-like sampling computations simple, Ann. Math. Statist. 27(1958), 37-54.

[11] Wishart, J.; Moment coefficient of the $k$-statistics in samples from a finite population, Biometrika 39 (1952) 1-13.

[12] Yamakawa, N.; Random combined fractional factorial designs, 1, Bull. Math. Statist. 11, No. 3-4, 1-38. 